\title{
Roadmap on optical metamaterials
}

Augustine M. Urbas ${ }^{1,24}$, Zubin Jacob ${ }^{2,3}$, Luca Dal Negro ${ }^{4}$, Nader Engheta ${ }^{5}$, A.D. Boardman ${ }^{6}$, P. Egan ${ }^{6}$, Alexander B. Khanikaev ${ }^{7}$, Vinod Menon ${ }^{8}$, Marcello Ferrera ${ }^{3,9}$, Nathaniel Kinsey ${ }^{3}$, Clayton DeVault ${ }^{3}$, Jongbum Kim ${ }^{3}$, Vladimir Shalaev ${ }^{3}$, Alexandra Boltasseva ${ }^{3}$, Jason Valentine ${ }^{10}$, Carl Pfeiffer ${ }^{11}$, Anthony Grbic ${ }^{11}$, Evgenii Narimanov $^{3}$, Linxiao Zhu ${ }^{12}$, Shanhui Fan ${ }^{12}$, Andrea Alù ${ }^{13}$, Ekaterina Poutrina ${ }^{1}$, Natalia M. Litchinitser ${ }^{14}$, Mikhail A. Noginov ${ }^{15}$, Kevin F. MacDonald ${ }^{16}$, Eric Plum ${ }^{16}$, Xiaoying Liu ${ }^{17}$, Paul F. Nealey ${ }^{17}$, Cherie R. Kagan ${ }^{18}$, Christopher B. Murray ${ }^{18}$, Dorota A. Pawlak ${ }^{19,20}$, Igor I. Smolyaninov ${ }^{21}$, Vera N. Smolyaninova ${ }^{22}$, and Debashis Chanda $^{23}$

\section{Affiliations}

1) Materials and Manufacturing Directorate, Air Force Research Laboratory, Wright Patterson Air Force, Base, Ohio 45433, USA

2) Department of Electrical and Computer Engineering, University of Alberta, Edmonton, AB T6G 2V4, Canada

3) Birck Nanotechnology Center, School of Electrical and Computer Engineering, Purdue University, West Lafayette, IN 47906, U.S.A.

4) Department of Electrical and Computer Engineering \& Photonics Center, and Division of Materials Science and Engineering, Boston University, 8 Saint Mary Street, Boston, Massachusetts 02215, United States

5) Department of Physics and Department of Electrical and Systems Engineering, University of Pennsylvania, Philadelphia, Pennsylvania 19104, USA

6) Joule Physics Laboratory, Institute for Materials Research, University of Salford, Manchester, M5 4WT, UK

7) Queens College and The Graduate Center of The City University of New York, Queens, New York 11367, USA

8) City College and The Graduate Center of The City University of New York, Queens, New York 11367, USA

9) School of Engineering and Physical Sciences, Heriot-Watt University, David Brewster Building, Edinburgh, Scotland EH14 4AS, UK

10) Department of Mechanical Engineering, Vanderbilt University, Nashville, Tennessee 37212, USA

11) Department of Electrical Engineering and Computer Science, University of Michigan, Ann Arbor, Michigan 48109-2122, USA

12) Department of Electrical Engineering, and Department of Applied Physics, Stanford University, Stanford, California 94305, United States

13) Department of Electrical and Computer Engineering, University of Texas at Austin, Austin, Texas 78712, USA

14) Department of Electrical Engineering, University at Buffalo, The State University of New York, Buffalo, New York 14260, USA

15) Center for Materials Research, Norfolk State University, Norfolk, Virginia 23504, United States

16) Optoelectronics Research Centre and Centre for Photonic Metamaterials, University of Southampton, SO17 1BJ,UK

17) Institute for Molecular Engineering, University of Chicago, 5747 South Ellis Avenue, Chicago, Illinois 60637, USA

18) Department of Materials Science and Engineering, and Department of Chemistry, University of Pennsylvania, Philadelphia, Pennsylvania 19104, United States

19) Institute of Electronic Materials Technology, ul. Wolczynska 133, 01-919 Warsaw, Poland

20) Centre of New Technologies University of Warsaw, ul. Banacha 2C, 02-097 Warsaw, Poland.

21) Department of Electrical and Computer Engineering, University of Maryland, College Park, MD 20742, USA

22) Department of Physics Astronomy and Geosciences, Towson University, 8000 York Rd., Towson, MD 21252, USA

23) NanoScience Technology Center and College of Optics and Photonics (CREOL), University of Central Florida, Orlando, Florida 32826, USA 


\author{
Email: augustine.urbas@us.af.mil \\ ${ }^{24}$ Guest editor of the roadmap
}

\begin{abstract}
Optical metamaterials have redefined how we understand light in notable ways: from strong response to optical magnetic fields, negative refraction, fast and slow light propagation in zero index and trapping structures, to flat, thin and perfect lenses. Many rules of thumb regarding optics, such as mu $=1$, now have an exception, and basic formulas, such as the Fresnel equations, have been expanded. The field of metamaterials has developed strongly over the past two decades. Leveraging structured materials systems to generate tailored response to a stimulus, it has grown to encompass research in optics, electromagnetics, acoustics and, increasingly, novel hybrid materials responses. This roadmap is an effort to present emerging fronts in areas of optical metamaterials that could contribute and apply to other research communities. By anchoring each contribution in current work and prospectively discussing future potential and directions, the authors are translating the work of the field in selected areas to a wider community and offering an incentive for outside researchers to engage our community where solid links do not already exist.
\end{abstract}




\section{Contents}

1) Introduction

\section{New materials capabilities and concepts}

2) Virtual photon interactions mediated by metamaterials

3) Ways to aperiodic metamaterials

4) Mathematical operations and processing with structured materials

5) New horizons for hyperbolic metamaterials

6) Topological effects in metamaterials

\section{Control of surface properties}

7) Functionally doped metal oxides for future ultra-fast active metamaterials

8) Optical dielectric metamaterials and metasurfaces

9) Beam shaping with metasurfaces

10) Control of emission and absorption with metamaterials

11) Control of far-field thermal emission properties through the use of photonic structures

\section{Nonlinear, active and tunable properties}

12) Engineering mid-infrared and optical nonlinearities with metamaterials

13) Directional control of nonlinear scattering from metasurfaces

14) Nonlinear properties of optical metamaterials

15) Optical gain in plasmonics and metamaterials

16) Coherent control in planar photonic metamaterials

17) Nanomechanical photonic metamaterials

\section{Assembly at scale}

18) Self-assembly and directed assembly for the fabrication of optical metamaterials

19) Nanoparticles for complex multimaterial nanostructures

20) Eutectics as metamaterials, plasmonic materials and photonic materials

21) Self-assembled hyperbolic metamaterials

22) Large area, roll-to-roll processing of optical metamaterials 


\section{Introduction - Augustine M. Urbas \\ Air Force Research Laboratory}

Optical metamaterials focuses on meso-structured multimaterial systems which give an effective response to optical fields that is different from the component materials. The large and active community of optical metamaterials researchers has reviewed the field regularly to capture exciting results and the current state of the art with notable reviews on assembly strategies [1,2], optical devices [3,4], nanoscale fields and properties manipulation [5-8] as well as controllable [9] and nonlinear optical properties [10]. In an effort to present emerging fronts in areas of optical metamaterials that could contribute and apply to other research communities, the authors of this roadmap translate the work in a selected field to a broader audience.

The roadmap is divided into 16 sections, each written from the perspective of a prominent researcher and that emphasizes one theme under the umbrella of optical metamaterials. In a community as sizable and diverse as metamaterials, a comprehensive view is impractical in this format. However, each article, by bridging current work and future developments, will provide some insight to the reader and orient them to a significant feature in the metamaterials community. As in any map, these features and the relation between them define the landscape. In this way, the roadmap will position current optical metamaterials work within the larger scientific community and provide researchers awareness of developments and possible connections between fields in order to facilitate the incorporation of optical metamaterials as an engineering approach and foster new scientific connections.

This roadmap will focus on materials systems with effective responses at the wavelength scale which display engineered linear and nonlinear optical properties. These structured metal and dielectric systems leverage the properties of parent materials, but yield characteristically different effects. Their use is not without challenges however as losses and dispersion often accompany optical metamaterials as well as the difficulty of fabricating high precision subwavelength structures. This has not dissuaded people from continuing to develop and explore novel configurations and responses in optical metamaterials. As the field and community of researchers grow, a natural expectation is that there will be a technological relevance yielded by these new insights and tools. Based on what has occurred in RF and microwave metamaterials, the development of technology rooted in research on metamaterials occurs when the unique properties and attributes of these artificial materials systems are tailored to meet the needs of some specific application. In this way, metamaterials are not generally useful, but can be specifically configured to provide an engineered response when exotic, textured, or dynamic properties are needed. As a discipline, metamaterials has extended the engineering space to incorporate the very nature of the response to incident fields. Where historically materials response has been explored and utilized, it can now, in some cases, be designed. The exploration and adoption of this capability by the wider engineering community is the next step for the application of optical metamaterials and this requires a greater communication between engineers and scientists inside and outside of the metamaterials community. It is notable that early maps of America made by native peoples and settlers did not contain reference to each other's settlements, borders and travel routes when describing the same land. Scientific communities can display a similar lack of mutual awareness even in aligned and overlapping areas. This roadmap can serve the purpose of anchoring the frontiers of metamaterials research to collateral work from adjacent communities. As well, it will highlight the techniques developed and employed in metamaterials which may provide solutions in different fields. Perhaps most importantly, the roadmap will explore the new growth in optical metamaterials where further refinements and novel capabilities can provide revolutionary advances in science and technology.

\section{References}

[1] Fontana, Jake, and Banahalli R. Ratna. "Toward high throughput optical metamaterial assemblies." Applied Optics 54, no. 31 (2015): F61-F69.

[2] Fruhnert, M., S. Mühlig, F. Lederer, and C. Rockstuhl. "Towards negative index self-assembled metamaterials." Physical Review B 89, no. 7 (2014): 075408.

[3] Yu, Nanfang, and Federico Capasso. "Flat optics with designer metasurfaces." Nature materials 13, no. 2 (2014): 139-150.

[4] Fleury, Romain, and Andrea Alù. "Cloaking and invisibility: A review." In Forum for Electromagnetic Research Methods and Application Technologies (FERMAT), vol. 1, no. 7, pp. 1-24. 2014.

[5] Ross, Michael B., Martin G. Blaber, and George C. Schatz. "Using nanoscale and mesoscale anisotropy to engineer the optical response of three-dimensional plasmonic metamaterials." Nature communications 5 (2014). [6] Pendry, John B. "Controlling Light on the Nanoscale (Invited Review)." Progress In Electromagnetics Research 147 (2014): 117-126.

[7] Soukoulis, Costas M., and Martin Wegener. "Past achievements and future challenges in the development of three-dimensional photonic metamaterials." Nature Photonics 5, no. 9 (2011): 523-530.

[8] Schuller, Jon A., Edward S. Barnard, Wenshan Cai, Young Chul Jun, Justin S. White, and Mark L. Brongersma. "Plasmonics for extreme light concentration and manipulation." Nature materials 9, no. 3 (2010): 193-204. [9] Turpin, Jeremiah P., Jeremy A. Bossard, Kenneth L. Morgan, Douglas H. Werner, and Pingjuan L. Werner. "Reconfigurable and tunable metamaterials: a review of the theory and applications." International Journal of Antennas and Propagation 2014 (2014).

[10] Lapine, Mikhail, Ilya V. Shadrivov, and Yuri S.

Kivshar. "Colloquium: nonlinear metamaterials." Reviews of Modern Physics 86, no. 3 (2014): 1093. 


\section{Virtual photon interactions mediated by metamaterials - Zubin Jacob \\ Purdue University and University of Alberta}

\section{Status}

Noise is omnipresent. Thermal noise inside materials is the fundamental origin of black body radiation while quantum noise in vacuum lies at the heart of exotic phenomena like the Lamb shift and Casimir forces. Recently, the field of metamaterials has started addressing a fundamentally new area: control of thermal and quantum fluctuations. A unique unified perspective is emerging on how artificial materials can alter and control the zero point and thermal energy density inside materials for a range of applications including coherent thermal sources, thermophotovoltaics, non-classical light sources and enhanced quantum interactions [1-5].

It is quite underappreciated that Rytov's seminal work on analyzing fluctuations inside matter can fruitfully be applied to artificial nanostructured media. A few research groups are spearheading the activity to identify experimentally observable consequences of enhanced quantum and thermal noise in metamaterials. However, care has to be taken to include the role of losses, dispersion and finite unit cell sizes and it is quite easy to run into incorrect conclusions. For example, quantum noise exists for all frequencies from zero frequency to infinitely large frequencies and it is naive to expect a metamaterial can alter this noise in an infinite bandwidth. Yet, there is a subset of fluctuational effects that can be enhanced by metamaterials. Resonant fluctuational energy and resonant fluctuational forces take unique functional forms inside metamaterials and can surely be engineered in realistic systems [2]. Hyperbolic and Chiral metamaterials are the front-runners in this area of research due to the advancements which have been made in their fabrication and robust characterization [2].

\section{Current and Future Challenges}

1) Long-range virtual photon interactions: One of the fundamental interactions in nature is dipole-dipole electromagnetic interactions which mediates Casimir forces between macroscopic bodies, Casimir-Polder force between an atom and a body as well as Van der waals interaction between two neutral ground state atoms [1]. It is extremely difficult to engineer these forces due to their short-range nature and contribution from virtual photons of infinite bandwidth [1]. One major future direction for the field is in the engineering of virtual photons which are known to obey a coulombic-interaction law in the near-field. The exchange of virtual photons between atoms or

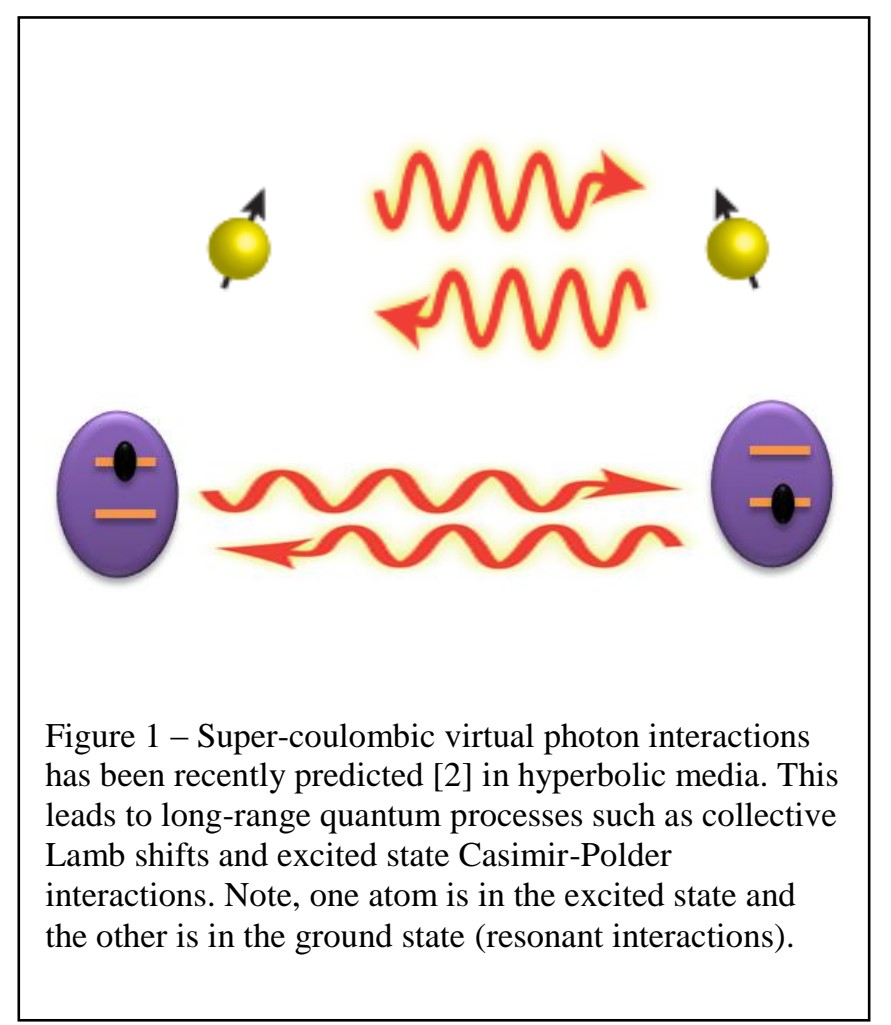

molecules can have measurable consequences such as the collective lamb shift of energy levels between multiple atoms. If the metamaterial can be engineered to alter this virtual photon interaction, many quantum electrodynamic effects can be enhanced even at large inter-molecular distances. The fore-runner in the achievement of such long-range super-Coulombic [2] virtual photon effects are hyperbolic and epsilon-nearzero metamaterials $[3,4]$. Long-range virtual photon interactions can induce entanglement between distant atoms and also help address the long-standing issue of scalability of qubits. Theoretical work has recently come to light [2-5] and there are multiple experimental efforts on-going to verify whether such theoretical predictions can be fruitfully materialized.

2) All-dielectric metamaterials for transparent subdiffraction photonics: One important issue that has to be overcome for the field of metamaterials is losses. One of the most promising approaches to address this issue is to use completely transparent building blocks to construct all-dielectric metamaterials. However, it is commonplace belief that all-dielectric transparent media can never confine light better than the diffraction limit. In a recent paper, we showed that a non-intuitive design of an all-dielectric metamaterial cladding can help beat the diffraction limit with no metal [6,7]. The cladding is made of a unique transparent meta-crystal that allows for total internal reflection but simultaneously acts on the evanescent waves to compress their extent in the cladding. This waveguide is called an extreme skin-depth waveguide since it engineers the skin-depth of evanescent waves 
in the cladding. Note no naturally occurring medium has the required property of providing total internal reflection while simultaneously engineering evanescent waves and can only be done using all-dielectric metamaterials.

3) Negative frequency resonance and Giant Vacuum Friction: Along with technological breakthroughs, the fundamental understanding of moving media and quantum vacuum can also be advanced due to the efforts in the field of metamaterials. Many elusive effects such as vacuum friction, the drag force that vacuum fluctuations exert on moving media have not been measured or understood in detail till date. We have discovered an interesting case where coupling can occur between plasmonic modes with positive and negative frequency in moving media which leads to a unique singular resonance $[8,9,10]$. An experimental scenario where this can occur is if two metallic plates separated by a small gap are in motion relative to each other. A wave can appear as a positive photonic mode on one plate and be Doppler-shifted to a mode of equal - but negative frequency - on the other plate if the velocity of the plates and the size of the gap between them both have critical values. This situation gives rise to perfect coupling of waves of positive and negative frequency in the near-field region and a resonance with an infinite quality factor, which is fundamentally different from any resonance in stationary systems [8]. This resonance leads to giant vacuum friction and is an important quantum phenomenon which can occur at the nanoscale [8].

\section{Advances in Science and Technology to Meet Challenges}

One of the most important advancement needed to measure quantum interactions is precise positioning of single quantum emitters. This is a challenging task but can be undertaken with atomic force microscope based positioning. Another important challenge for the field is addressing losses and this can be done using alldielectric metamaterials based on silicon. All-dielectric media can be explored along host of interesting fundamental directions including quantum and thermal photonics and it can pave the way also for practical metamaterial devices.

\section{Concluding Remarks}

In conclusion, this roadmap outlines two important ideas on engineering virtual photons using metamaterials and the rising field of all-dielectric metamaterials. Virtual photon engineering is a fertile playground for a host of new fundamental scientific

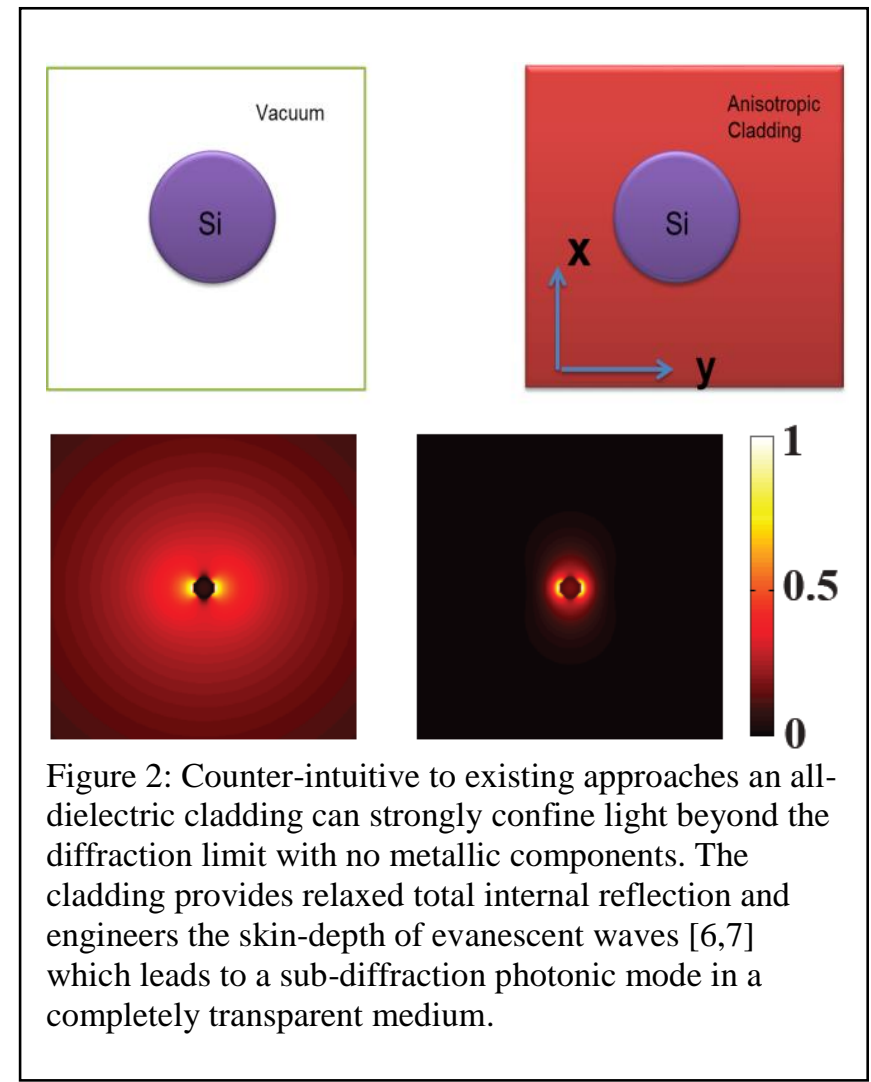

ideas and all-dielectric media can have the transformative impact on technology that the field of metamaterials has promised.

References

[1] Milonni, Peter W. The quantum vacuum: an introduction to quantum electrodynamics. Academic press, 2013.

[2] C. Cortes and Z. Jacob, arXiv:1601.04013, 2016

[3] Sokhoyan, Ruzan, and Harry A. Atwater. arXiv preprint arXiv:1510.07071 (2015).

[4] Alù, Andrea, and Nader Engheta. Physical review letters 103.4 (2009):

[5] Milton, Graeme W., Ross C. McPhedran, and Ari Sihvola. Optics express 21.12 (2013): 14926-14942.

[6] S Jahani, Z Jacob Nature nanotechnology 11 (1), 23-36

[7] S Jahani, Z Jacob Optica 1 (2), 96-100

[8] Y Guo, Z Jacob Optics express 22 (21), 26193$26202(2014)$

[9] MG Silveirinha, Phys. Rev. X 4 (3), 031013 (2014)

[10] Pendry, J. B. Journal of Physics: Condensed Matter 9.47 (1997): 10301. 


\section{Ways to aperiodic metamaterials - Luca Dal Negro \\ Boston University}

\section{Status}

Photonic metamaterials are artificially engineered media with unusual and intriguing properties that emerge from the interaction of electromagnetic radiation with their sub-wavelength components. Metamaterials are typically made of two different building blocks periodically arranged with a small period compared to the optical wavelength, such that they can be regarded as artificial homogenized media. For example, metamaterials can exhibit negative permittivity $\varepsilon$ and/or permeability $\mu$, negative refractive index, near-zero $\varepsilon$ values (i.e., ENZ media) or zero (volume) averaged refractive index regions resulting with non-Bragg optical gaps. Due to the subwavelength nature of their components, it is commonly believed that metamaterials are accurately described within the standard Effective Medium Theory (EMT). However, this approach is correct only when considering their local response, and it can fail completely at high frequency (i.e., optical) in the case of sub-wavelength structures with structurally complex geometries, such systems with aperiodic positional order ${ }^{1}$, especially at optical frequencies. A simple example is provided by one-dimensional metamaterials with layers arranged according to fractal generation rules or more general aperiodic sequences. The distinctive symmetries (e.g. scale-invariance symmetry, local parity/reflection symmetry, etc.) embedded in deterministic aperiodic sequences significantly affect the metamaterial properties by establishing structural correlations that give rise to complex (i.e., generally multifractal) distributions of transmission gaps of varying widths at all wavelengths, irrespective of the optical thickness of the component layers. In addition, periodic/aperiodic positional ordering of sub-wavelength building blocks invariably introduces nanoscale nonlocal effects. We will refer to this more general scenario as to "correlation metamaterials", meaning structures where positional order and correlation effects strongly influence their overall optical responses in the metamaterial limit. If properly engineered, nonlocal effects in such structures may result in a number of novel applications to nanoguiding and nonlinear signal generation especially with respect to anisotropic media with hyperbolic dispersion. However, nonlocal effects in deterministic aperiodic systems have only recently been addressed ${ }^{2}$, and it was shown that they are controlled by the inhomogeneous modulation of the coupling strength of surface plasmon polaritons that propagate along metaldielectric interfaces. These preliminary considerations suggest that correlation metamaterials designed according to aperiodic mixing rules can expand our horizon well-beyond the conventional EMT paradigm, providing access to additional degrees of freedom and opportunities. In particular, understanding the distinctive behavior of aperiodic metamaterials with structural resonances extending across multiple wavelength scales may advance fundamental science as well as engineering applications especially in the important areas of multiplexed optical bio-sensing and enhanced nonlinear frequency conversion. As we will briefly discuss in the next section, there are several ways to aperiodic metamaterials that already promise to overcome the current limitations of large loss/bandwidth ratio and weak magnetic responses (i.e., small permeability) at optical frequency.

\section{Current and Future Challenges}

There are multiple routes, not necessarily disconnected, that demonstrate the relevance of aperiodic order and positional correlations to metamaterials research. One path originates from the systematic numerical optimization of specific metamaterials functions, such as broadband nanofocusing, negative refraction, reduced optical losses, etc. Genetic optimization algorithms have been successfully applied to the plasmonic nano-antennas ${ }^{3}$ and negative-index metamaterials ${ }^{4}$, demonstrating optimal aperiodic designs. However, significant advances at developing more efficient and accurate electromagnetic simulation tools capable of dealing with device-level complexity, large electric size parameters and localized nonlinearities are needed in order to fully leverage these powerful optimization techniques. Alternative ways towards the engineering of aperiodic media are provided by digital ${ }^{5}$ and coding, or programmable, metamaterials 6 . According to these very powerful visions, it is possible to digitally synthesize optical structures that perform desired functions by properly sculpting only two material "bits" into local functional units called metamaterial bytes. Differently from traditional metamaterials, often constrained by log-range periodicity, digital metamaterials ${ }^{7}$ possess well-defined structural units (i.e., the bytes) simply designed by mixing two material bits at the nanoscale. As a result, carefully designed local spatial ordering contributes to determine the effective properties of digital metamaterials, analogously to ordered sequences of " $0 \mathrm{~s}$ " and " $1 \mathrm{~s}$ " that convey information by their local position within more complex digital messages (for more details see Nader Engheta's contribution in this Roadmap). On the other hand, in the related approach of coding metamaterials ${ }^{8}$ the resulting bytes do not even need to be described by effective medium parameters. Such metamaterials only rely on the 
presence of two types of unit cells with 0 and $\pi$ phase responses, which can be controlled by fieldprogrammable gate arrays (FPGAs). Desired optical properties can be achieved by "programming" two materials bits that display, when assembled into a specific pattern, the targeted responses. In particular, by dynamically programming the same metamaterial using different coding sequences it becomes possible to manipulate electromagnetic fields in very different manners and thus realize distinctly different functionalities. The third path to aperiodic order is the one of quasi-crystals and aperiodic tilings, here referred to as the "geometrical approach". This approach, which is rooted in geometry (e.g., fractal geometry and aperiodic point-patterns) has been largely investigated in nanophotonics where it led to the numerous device applications to optical sensing, nonlinear optics, light sources, energy harvesting and solar cells ${ }^{7}$. The geometrical approach leverages a large number of rigorous results on the relationship between the type of structural order displayed by a given system, its energy spectrum and wave transport properties. In the regime of metamaterials, enhanced transmission resonances through quasi-crystal arrays of sub-wavelength apertures was demonstrated ${ }^{8}$, as well as broadband transmission of $\mathrm{THz}$ radiation with enhanced efficiency through quasi-periodic grating structures 9 . Moreover, aperiodic patterns of $\mathrm{Ag}$ nanowires that exhibit strong magnetic resonance with negative effective index at optical frequency (even stronger than their periodic counterparts) have been recently demonstrated ${ }^{10}$. However, in order to drastically reduce the inherent optical losses of metalbased components, it is highly desirable to implement future devices using all-dielectric materials within a scalable and cost-effective fabrication approach. Work addressing this important problem is discussed in the Jason Valentine's contribution to this Roadmap.

\section{Advances in Science and Technology to Meet Challenges}

The engineering of all-dielectric aperiodic metamaterials with multi-scale structural resonances induced by positional correlations has the potential to enable more efficient devices with controllable responses over multi-spectral ranges, at reduced optical losses and featuring novel transport and scattering properties. However, in order to implement this vision, several technological advances in materials growth and device fabrication become now compelling: i) low-loss and silicon-compatible resonant materials need to be developed to replace noble metals in the near-infrared and optical regime. Conductive oxides and nitrides materials have been recently demonstrated with tunable dispersion properties for the engineering of linear $^{11}$ and nonlinear ${ }^{12}$ plasmonics and ENZ metamaterials, showing a great potential for highdensity device integration on silicon; ii) highthroughput and cost-effective fabrication techniques need to be developed that can equally accommodate periodic and aperiodic designs with deep subwavelength features over large areas. A related challenge that needs to be addressed in the near future is the three-dimensional fabrication of aperiodic nanostructures. If realized, these structures could additionally leverage strong chiral effects for the engineering of negative index and add new vistas to the powerful vision of digital metamaterials and transformation optics.

\section{Concluding Remarks}

In this section I have reflected on some of the recent developments that shine new light on aperiodic designs in metamaterials research. Understanding and exploiting aperiodic order in low-loss all-dielectric metamaterial structures with engineered non-local responses, geometrically determined electric/magnetic resonances, and enhanced nonlinearities can result in novel devices that operate across multiple spectral bands with unprecedented control of electromagnetic field localization and transport phenomena.

\section{References}

[1] L. Dal Negro Ed., Optics of Aperiodic Structures: Fundamentals and Device Applications, Pan Stanford Publishing, 2014.

[2] S. Savoia, et al., Optical nonlocality in multilayered hyperbolic metamaterials based on Thue-Morse superlattices, Phys. Rev. B., 87, 235116 (2013)

[3] C. Forestiere, et al., "Genetically optimized plasmonic nanoarrays", Nano Lett., 12, 2037 (2012);

[4] Y. Dong, S. Liu, "Topology optimization of patchtyped left-handed metamaterial configurations for transmission performance within the radio frequency band based on the genetic algorithm", $\mathrm{J}$. Opt. 14, 105101 (2012).

[5] C. Della Giavampaola, N. Engheta, Digital metamaterials", Nat. Mater., 13, 1115 (2014).

[6] T. J. Cui, et al, "Coding metamaterials, digital metamaterials and programmable metamaterials", Light: Science \& Applications, 3, e218 (2014).

[7] L. Dal Negro, "Optics of Aperiodic Structures: Fundamentals and Device Applications", Pan Stanford Publishing (2014).

[8] T. Matsui et al., "Transmission resonances through aperiodic arrays of subwavelength apertures", Nature, 449, 517 (2007)

[9] X. Ren et al., "Nonperiodic metallic gratings transparent for broadband terahertz waves", Phys. Rev. B., 91, 045111 (2015).

[10] A. Agrawal, et al., "Negative permeability with arrays of aperiodic silver nanoclusters", Appl. Phys. Lett., 101, 083109 (2012). 
4

5

6

7

8

9

10

11

12

13

14

15

16

17

18

19

20

21

22

23

24

25

26

27

28

29

30

31

32

33

34

35

36

37

38

39

40

41

42

43

44

45

46

47

48

49

50

51

52

53

54

55

56

57

58

59

60

[11] P. R. West, et al., "Searching for better plasmonic materials", Laser Photonics Rev. 4, 795 (2010)

[12] A. Capretti et al., "Enhanced third-harmonic generation in Si-compatible epsilon-near-zero indium tin oxide nanolayers", Opt. Lett., 40, 1500 (2015). 


\section{Mathematical operations and processing with structured materials - Nader Engheta University of Pennsylvania}

\section{Status}

Materials control waves, and as such they can, in principle, tailor, manipulate, redirect, and scatter waves at will. In the field of metamaterials, the unprecedented possibility to design and engineer materials with desired material parameters temporally, spatially, locally, and/or globally provides us with exciting opportunities to manipulate waves, e.g., electromagnetic and optical waves, in order to conduct useful functionalities in devices and components [1]. Examples of conventional optical devices made of traditional materials include ubiquitous thin lenses, optical fibers and filters, phase plates and polarizers, and modulators and detectors, to name a few. However, metamaterials can bring a new vista for the next generation of wave-based functional nanostructures. For example, can we envision structured materials at the nanoscale that may deform wave profiles in order to perform mathematical operations and computation, such as differentiation, integration, convolution, etc. on incoming wave profiles? Why should such nanostructures be useful and important? We live in a world where communications and interaction among people require huge amounts of data bandwidth to support online interaction and social media. Handling such audio and video data transmission and storage in a shrinking volume and an increasing speed demands a new mindset and a disruptive methodology and approach. Metamaterials may offer an attractive possibility for a roadmap to address such a challenge. Recently, our team [2] proposed the ideas for such "computational metamaterials" with possibility to perform mathematical operations on incoming wave's profiles. This may potentially lead to a new paradigm for mathematical computation and signal processing with unprecedented speeds. This was a natural evolution of the earlier idea of optical metatronics we introduced several years ago $[3,4]$, in which we unified the alphabets of electronics and photonics. Specifically, in that work we explored how deeply subwavelength nanostructures, made of properly selected materials, interact with light as "lumped optical circuit elements at the nanoscale", such as optical nanoinductors, optical nanocapacitors, optical nanoresistors, etc. [3, 4]. Just as in the electronic circuitry, where lumped circuit elements are connected in order to construct functional circuits, here in our optical metatronic circuit paradigm collections of properly arranged nanostructures may also function as optical nanocircuits with essentially analogous functionalities. Experimental realizations of such optical metatronic

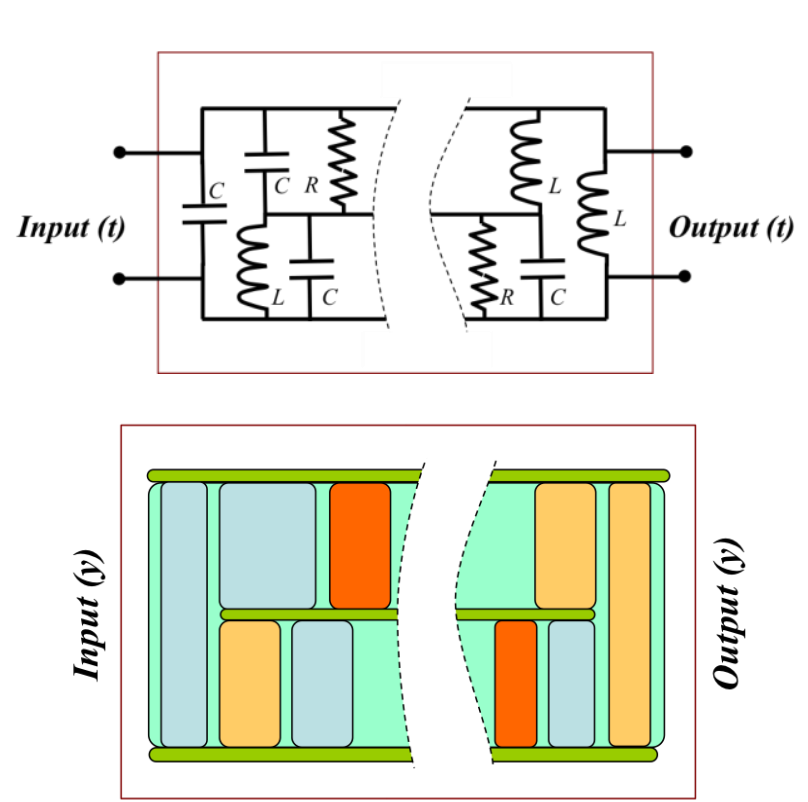

Figure 1 - Sketches of (Top) an electronic processor, formed by a collection of electronic circuit elements; and (Bottom) an optical metatronic processor, formed by various structured material blocks.

circuits have also been performed subsequently [5-7], demonstrating that indeed we can synthesize circuits with light at the nanoscale.

\section{Current and Future Challenges}

Conventional digital computers, which are an indispensible part of our daily lives, are formed by clusters of numerous electronic "circuit elements", functioning based on sophisticated algorithms and processing methodologies. Although these computers are already amazingly fast, handling growing amounts of data begs for new transformative ideas for future computing platforms that are faster in speed, smaller in volume, and lower in power. It is interesting to explore whether optical metatronics may be a candidate for such a platform. Figure 1 shows the analogy between the electronic signal processing and its optical metatronic counterpart. In electronics, a collection of circuits provides a processor with a desired transfer function affecting the temporal frequency contents of incoming signals (Fig. 1a). By analogy, the optical metatronic processor will be made of collections of nanomaterial structures, each acting as an optical circuit element but when arranged together function with a given spatial (and may also be temporal) transfer function (i.e., desired spatial Green function) operating on an incoming wave, resulting in an output wave, whose spatial profile resembles the result of the desired mathematical operation on the profile of the incident wave. In our recent work [2], our team has offered two possible approaches for such metamaterial wave-based processors: (1) to design metasurfaces with required transfer functions in the spatial Fourier domain; (2) to engineer desired Green 
functions directly in the spatial coordinate domain, bypassing the need to perform spatial Fourier transform. In the former approach, one needs to utilize optical structures that perform spatial Fourier transform (such as conventional convex lenses or graded-index dielectric slabs and fibers) before adding the metasurfaces with desired transfer function (e.g., $i k$ in the wavenumber $k$ domain for the operation of spatial differentiation in the spatial coordinates), while in the latter there is no need for the spatial Fourier transformation, and thus much thinner structures such as multilayered metamaterial structures with thickness of the order of a few wavelengths can be designed to engineer the needed Green function. The details of these two approaches can be found in [2]. The idea of "metamaterial analog computing" and "wave-based signal processing" by exploiting wave interactions with combinations and collections of material nanostructures may provide a transformative direction in signal processing at the nanoscale and is one of the exciting directions for the field of metamaterials. This idea may also be augmented with the notion of "digital metamaterials" [8], in which two constituent material building blocks as "metamaterial bits" can be combined to achieve a desired set of material parameters. As in any field, there are of course challenges ahead. These include the need for highprecision nanofabrication, highly accurate characterization of material parameters, and structural and material stability in temperature-varying environments, just to name a few. Moreover, such a wave-based processing platform will tremendously benefit from programmable architectures via reconfigurable materials.

\section{Advances in Science and Technology to Meet Challenges}

Challenges mentioned above will likely be confronted and addressed with more sophisticated nanofabrication technology and precision characterization methodologies. The next generation of the near-field scanning optical microscopy (NSOM) with multiprobe systems, deeply subwavelength nanoscopy, and other electron-beam-based characterization techniques such as cathodoluminescence (CL) microscopy and electron-energy-loss spectroscopy (EELS) are all useful tools to address the need for future nanomaterial characterization and metrology. Another important element in the path to achieving "wave-based analog computing" using metamaterials is the possibility for tunability and reconfigurability of constituent materials elements forming such devices in order to perform different operations with the same material device. In integrated electronic circuits, the notion of "fieldprogrammable gate arrays (FPGA)", in which a user can program the circuit after construction in order to

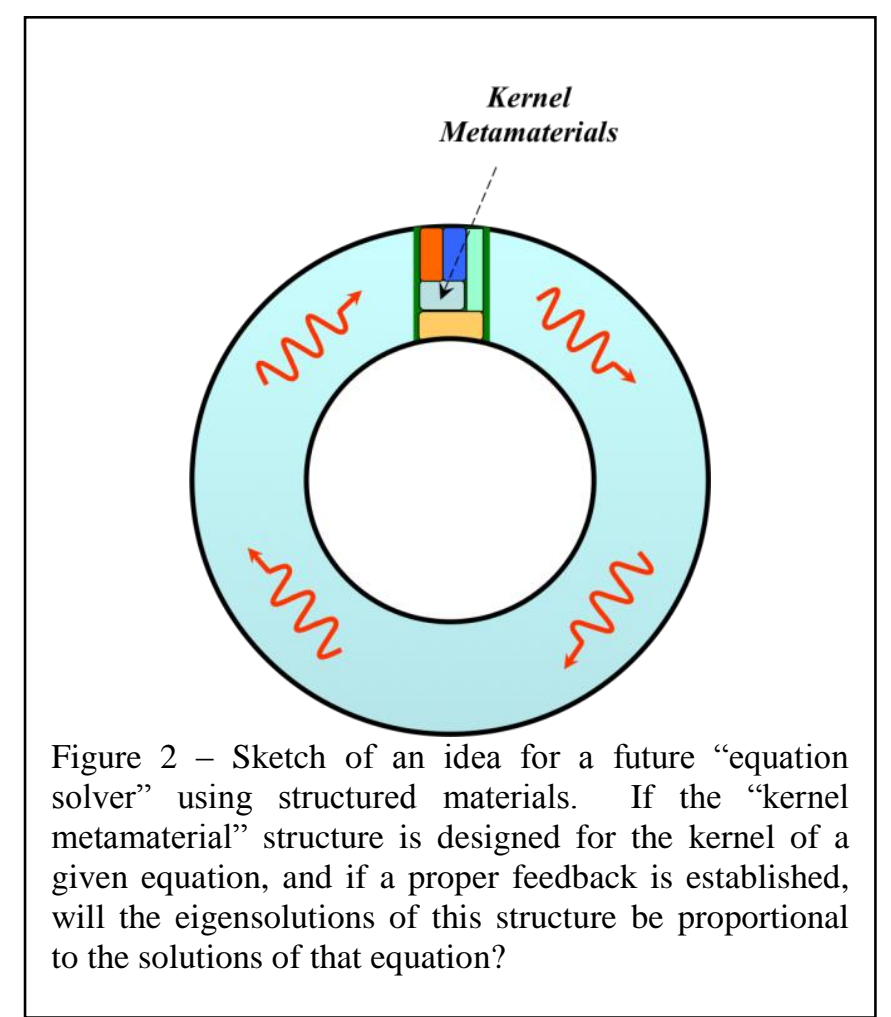

reconfigure it for a different functionality, has been proven to be a powerful concept in various applications of electronics [9]. It will be of great interest, if such a concept and its appropriately modified version, can be brought into the field of metamaterials. An example of a programmable metamaterial can be found in [10]. If FPGA-inspired metamaterials becomes a reality in the near future, numerous future applications will emerge in the field of nanophotonics. For example, one can envision "programmable light-based analog computing at the nanoscale", which can perform different mathematical operations by reconfiguring its material constituents at will. Finally, one of the exciting future directions following wave-based analog computing will be to explore how such a system can be augmented to "solve equations". If we start with the metamaterial structures that perform a mathematical operation such as differentiation or integration (e.g., the ones in [2]), by applying a proper feedback, may one be able to form an optical structure whose eigensolutions may be proportional to the solutions of a given differential or an integral equation? Figure 2 depicts this idea. Work is under way to explore the feasibility of these ideas.

\section{Concluding Remarks}

The field of metamaterials has seen fascinating development over the past decades, and new vistas and exciting directions are appearing in the horizon. The concept of "informatic metamaterials", briefly summarized here, may be one of the fruitful paths in the roadmap of metamaterials, providing a transformative platform for mathematical computation and information processing at the nanoscale. 


\section{References}

[1] W. Cai, V. Shalaev, Optical Metamaterials: Fundamentals and Applications, New York: Springer (2010)

[2] A. Silva, F. Monticone, G. Castaldi, V. Galdi, A. Alu, N. Engheta, "Performing Mathematical Operations with Metamaterials," Science, 343, 160163 (2014)

[3] N. Engheta, "Circuits with Light at Nanoscales: Optical Nanocircuits Inspired by Metamaterials", Science, Vol. 317, pp. 1698-1702 (2007).

[4] N. Engheta, A. Salandrino, A. Alu, "Circuit Elements at Optical Frequencies: Nano-Inductor, Nano-Capacitor, and Nano-Resistor," Phys. Rev. Lett. 95, 095504 (2005).

[5] Y. Sun, B. Edwards, A. Alu, and N. Engheta, "Experimental Realization of Optical Lumped Nanocircuit Elements at Infrared Wavelengths," Nature Materials, 11, 208-212 (2012).

[6] H. Caglayan, S.-H. Hong, B. Edwards, C. Kagan, and N. Engheta, "Near-IR Metatronic Nanocircuits by Design," Phys. Rev. Lett, 111, 073904 (2013).

[7] J. Shi, F. Monticone, S. Elias, Y. Wu, D. Ratchford, $\mathrm{X}$. Li, and A. Alu, "Modular assembly of optical nanocircuits," Nature Communications, 5:3896 (2014).

[8] C. Della Giovampaola and N. Engheta, "Digital Metamaterials," Nature Materials, 13(12), 11151121 (2014).

[9] C. Maxfield, FPGA: Instant Access, Burlington: Elsevier (2008).

[10] T. J. Cui, M. Q. Qi, X, Wan, J. Zhao, Q. Cheng, "Coding metamaterials, digital metamaterials, and programming metamaterials," Light: Science \& Applications, 3: e218 (2014). 


\section{New horizons for hyperbolic} metamaterials - A.D. Boardman and P. Egan University of Salford

\section{Status}

Metamaterials involve a fascinating range of artificial effective media, and the investigation of uniaxial nonmagnetic metamaterials is a strongly growing global activity. Such materials have a direction around which rotational symmetry is apparent. This direction is called the optic axis, shown in figure 1 . If the optic axis is along the $z$-axis of a standard rectangular coordinate system, then the permittivity tensor is diagonal.

If the tensor component, associated with the $z$-axis, is positive then the surface of constant frequency in wave number space is spherical for ordinary waves and elliptical for extraordinary waves. The interesting aspect is what happens when this diagonal element of the permittivity tensor becomes negative. The surface of constant frequency then becomes hyperbolic. This type of metamaterial is a non-magnetic dielectric and is, in principle, much easier to fabricate than the early metamaterials, where both the permittivity and the permeability are negative.

Ignoring the role of the optic axis orientation can give rise to lost design opportunities, and missing the ultimate revelation of dramatic new and unexpected pathways.

Even in the linear domain, the latter have, by no means, been extensive. It is, therefore, a very important field of study, and it can be anticipated that the exploration of the uniaxial symmetry properties of hyperbolic metamaterials will lead to a number of important, and exciting, applications.

Wave propagation in both linear and nonlinear uniaxial materials has been addressed many years ago ${ }^{1,2,3}$ but not in any metamaterial context. This historical work, however, certainly provides excellent groundwork, for the creation of a new roadmap for hyperbolic metamaterials. For other uniaxial metamaterials, it has already been pointed out in a pioneering publication that ignoring general optic axis orientations can lead to a false conclusion ${ }^{4}$. In spite of this, the need for a roadmap is made abundantly clear by the fact that there is very little in the literature of metamaterials about optic axis issues. Nevertheless, an excellent start has been made by some important recent work ${ }^{5,6,7}$ investigating metallic nanotubes, tilted with respect to any slab interfaces that may exist. This work involves waves in what has been called asymmetric hyperbolic media.

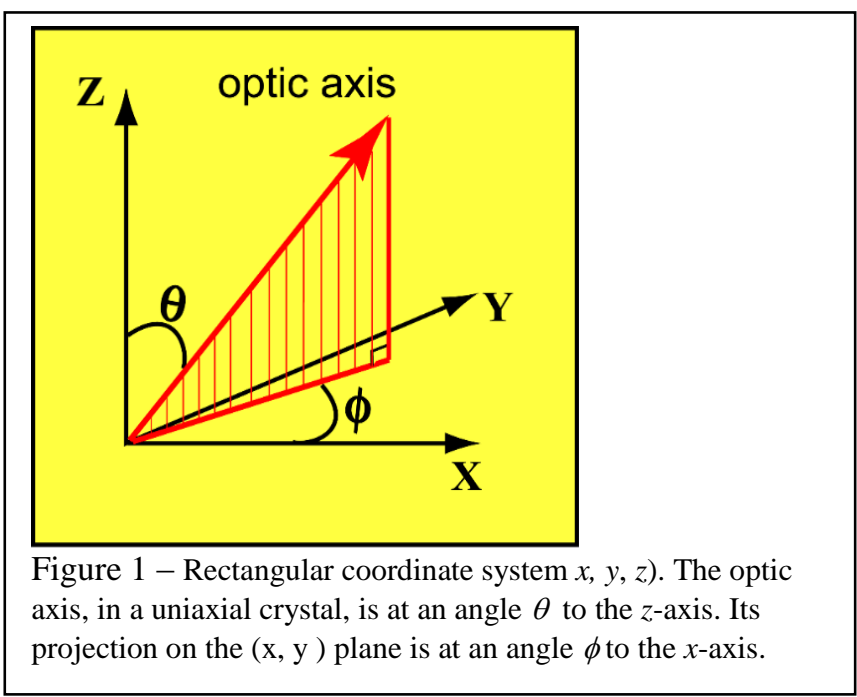

\section{Current and Future Challenges}

Given that there are some strong investigations in the area of linear, electromagnetic wave propagation in uniaxial metamaterials, the new challenge is to create a comprehensive theory that will reveal all the new horizons for hyperbolic metamaterials. The strong signpost that new and vital features rapidly develop, when optic axis positions are varied, emerged some years ago ${ }^{4}$ for uniaxial metamaterials that exhibit both dielectric and magnetic properties. Currently, the general impact of the optic axis position upon the behavior of hyperbolic metamaterials is also emerging ${ }^{8}$ but much more needs to be done. The motivation behind developing this roadmap of optic axis influence upon design and experiment is very strong. This is so much so that it is already known that ignoring anisotropy in metamaterial designs can lead to experimental expenditure being wasted.

In order to set up an investigation of how the orientation of the optic axis affects wave propagation in hyperbolic metamaterials, it is going to be necessary to use completely new forms of transformation optics? This must be captured in very modern, and highly effective, simulations. Although some examples of optic axis tilting do exist ${ }^{5,6,7}$, it is necessary to reemphasize that a complete roadmap exemplified by attractive pathways to optic-axis-driven work needs to be in place.

The roadmap required here will lead to downstream design changes that will, broadly, feature dividing its pathways into two classes. Class 1 will involve the interrogation of a slab of metamaterial, such as developing the transmission of light through an interface, or a set of interfaces, with the role of the optic axis being a fundamental control factor. Class 2 will involve guided waves dependent, in a critical fashion, upon special characteristics of hyperbolic metamaterials. The latter can be organized into a variety of geometries, for which the optic axis is 
allowed to adopt a number of interesting, and, yet, practical, positions. A start on the general, tensordriven, linear approach has been published ${ }^{8}$, but much more needs to be developed. A good example of the kind of fascinating outcome to be expected is shown in figure 2. Here a thin film planar guide, is investigated, which can be a hyperbolic metamaterial, or just anisotropic, supporting homogeneous waves. The orientation of the optic axis, using the angles defined in figure 1 , is a significant determinant of the resulting optical properties.

For conventional anisotropic waveguides homogeneous propagating waves occur over a range of $\theta$ that shows little change ${ }^{8}$ with the azimuthal angle $\phi$. For a hyperbolic guide, in which $\varepsilon_{3 f}<0$, figure 2 shows, quite dramatically, that there exists a critical angle $\phi_{c}$ exists, at which homogeneous waves propagate for all $\theta$.

This kind of outcome has significant implications for metamaterial waveguide designs because it implies that, in the hyperbolic case, after finding the correct azimuthal angle for the optic axis, generation of propagating modes can be more easily achieved, and the resulting structures are more tolerant to optic axis misalignment.

The importance of including nonlinearity into the modelling of hyperbolic metamaterials cannot be overemphasized. This is especially true when coupled to optic axis orientation. It is a major challenge for the future. In the entirely linear, loss-free, domain a number of windows of opportunity should emerge and point to really novel device designs, opening up for class 2 devices. It is, nevertheless, the role of nonlinearity that always remains as the strongest design challenge, in any area. This kind of impact of asymmetry upon the linear and nonlinear index was addressed, for non-metamaterials, some years ago $\mathbf{1 , 2 , 3}$ and that work, in itself, is clearly a useful roadmap guiding the new hyperbolic metamaterial research into a productive future.

\section{Advances in Science and Technology to Meet Challenges}

Since there is only a very limited literature on the role played by optic axis orientations in metamaterials, a really general theory is still required. The new pathways contained within the roadmap will need to bring together very advanced, tensor-driven mathematics that automatically folds in all aspects of symmetry theory. More dramatically, new transformation optics will be needed and, although there is already some base work ${ }^{9}$ on transformation

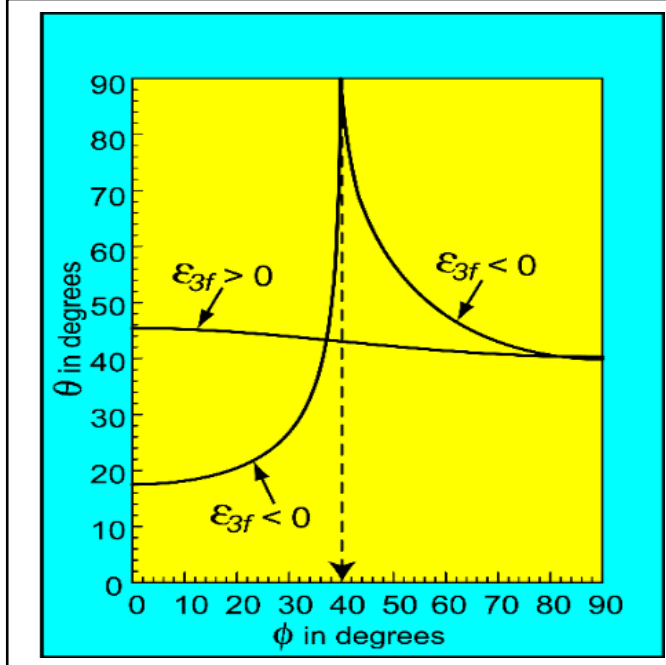

Figure 2 - Impact of optic axis orientation upon homogeneous guided wave range in a planar, uniaxial, thin film waveguide (f). The air cover (c) and the anisotropic substrate (s) are semi-infinite. The labels are the diagonal tensor element $\varepsilon_{3 f}$, associated with the $\mathrm{z}$ axis. Data : $\varepsilon_{3 s}=2.25, \quad \varepsilon_{1 f}=4, \quad\left|\varepsilon_{3 f}\right|=2.7889$ Range : $\theta=0^{\circ}$ up to each of the curves.

optics available on spacetime cloaks, ensuring that transformation optics involves asymmetry suitably mapped onto the nonlinear optics domain will not be a simple route to take. In spite of this aspect, new designs will be creatable using new technology pathways. The current major tool ${ }^{10}$ called COMSOL will need to be re-orientated, strongly, towards the work described above.

In general it is the opening and closing of important frequency windows, not previously anticipated in the published hyperbolic metamaterial literature, where the possibility of disturbances to the optic axis was ignored, that is a vital aspect of this roadmap. It is reemphasized here that planned optic axis manipulation brings in a new kind of wave physics that can be highly competitive with such obvious physical phenomena as absorption. A beautiful published comment on this is " ${ }^{4}$ "even small optic anisotropy is a much greater enemy of the perfect lens than absorption". This is an important encouragement and, tool-shaper, directing us to be very careful when looking at the roles of absorption, in the presence of an anisotropy.

\section{Concluding Remarks}

Small deviations from the expected position of the optic axis brings in new phase shifts, which end up dominating absorption. The pathways outlined here target asymmetric hyperbolic media, and this kind of asymmetry can involve an optic axis at any angle to the interface of a uniaxial crystal, so that the outcomes 
result in a new general theory. Also even modest amounts of power will initiate elegant nonlinear control, coupled to optic axis-driven designs.

The control of light propagating in complex asymmetric waveguides will be an immensely, important and interesting global area of activity. This will be directed towards the new horizons that will emerge from the kind of general theory revealed by the roadmap discussed here..

\section{References}

[1] Q. Guo and S. Chi "Nonlinear light beam propagation in uniaxial crystals: nonlinear refractive index, self-trapping and self- focusing" J. Opt. A: Pure and Appl. Opt. 2, 5 (2000).

[2] J. A .Fleck and M .D. Feit, "Beam propagation in uniaxial, anisotropic media” ,J.Opt.Soc.Am,73,920 (1983).

[3] A. Knoesen, T. K .Gaylord and M. G. Moharam, "Hybrid guided modes in uniaxial dielectric planar waveguides" Journal of Lightwave Technology,6, 1083 (1988).

[4] I. S Nefedov, C. A Valaginnopoulos and L. A. Melnikov, "Perfect absorption in graphene multilayers", J.Opt,.15,114003 (2013).

E. Starodubtsev, "Propagation and tunneling of electromagnetic waves through uniaxial metamaterials at arbitrary orientations of the optic axis", Proc. SPIE, 7355 (2009).

[5] S. M. Hashemi, I. S. Nefedov and M. Soleimani, "Waves in asymmetric hyperbolic media", Photonics Letters of Poland,.5, 72 ( 2013).

[6] I. S. Nefedov, C. A. Valagiannopoulos, S. M. Hashemi, and E. I. Nefedov, "Total absorption in asymmetric hyperbolic media", Scientific Reports, 3:2662,1 (2013)

[7] I. S. Nefedov, C .A. Valaginnopoulos and L. A. Melnikov, "Perfect absorption in graphene multilayers",J.Opt,.15,114003 (2013).

[8] A. D. Boardman. P. Egan and M. McCall, "Optic axis-driven new horizons for hyperbolic metamaterials", EPJ Applied Metamaterials, 2 (2015).

[9] M. McCall, A. Favaro, P. Kinsler and A. D. Boardman "A spacetime cloak, or history editor," J. Opt .13, 024003, 2011 
6. Topological effects in metamaterials Alexander B. Khanikaev and Vinod Menon The City University of New York

\section{Status}

One of the most recent developments in the field of metamaterials has utilized analogies with abstract topological concepts and ideas that have been shown to be fruitful in other branches of physics and material science, such as topological phase transitions and topological insulators. These recent advancements also pave a completely new direction for the field of metamaterials. One can envision novel unprecedented electromagnetic properties which can be achieved only by applying fundamental topological concepts. While there has been a plethora of topology inspired works recently, we focus on two representative examples: i) photonic topological insulators and ii) optical topological phase transitions.

Topological insulators [1], originally a condensed matter concept, represent an exotic state of quantum matter which cannot be characterized by symmetry alone and requires additional topological consideration for its complete understanding. In contrast to the wellknown conventional insulating and conducting states of material, the topological insulators are insulating in the bulk, but allow for electronic transport to occur on their surface in the form of edge states. However, the most peculiar property which has dramatic implications on electronic transport in topological insulators is the robustness of these edge states, which is intimately related to their topological origin. It appears that the edge state are characterized by their spin being in one-to-one correspondence with their propagation direction or "spin-locked", which makes these modes insensitive to local defects, disorder, and other structural imperfections. The latter property appears to be of immense value in electromagnetics, where an exotic state analogous to topological insulators was recently emulated with the use of magnetic photonic crystals [2,3] and bianisotropic metamaterials [4] and experimental confirmations of robustness observed [3,5,6,7]. As explained below, this may allow solving multiple technological issues the field of metamaterials is currently facing.

Another topological concept - optical topological transition (OTT) - emerges in hyperbolic metamaterials. These structures are known to be characterized by very unusual topology of their constant frequency contours, which, in contrast to elliptical contours found in conventional dielectrics, assume hyperbolic shapes. However, the most interesting behavior takes place at the crossover between elliptical and hyperbolic topologies (Fig. 2a) where the mathematical singularity at the transition

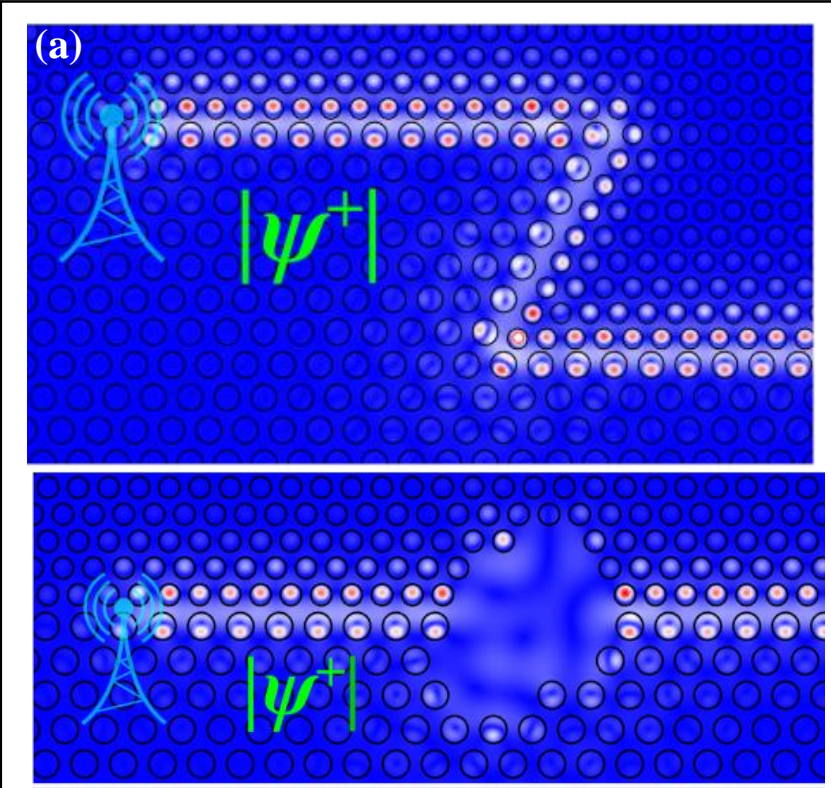

Fig. 1: Topologically protected propagation of light in photonic topological insulators (a) avoiding backreflection at sharp corners and (b) avoiding formation of Fabry-Perot resonances in the cavity.

point manifests itself as a singularity in the photonic density of states (PDOS) [8]. This phenomenon has been used to demonstrate large increase in spontaneous emission rate of a dipole emitter placed in the near field of the metamaterial. In addition a transition between these two distinct topologies can be induced in a very unusual way when an external magnetic field is applied, leading to strongly nonreciprocal response and a unidirectional or "one-way" character of the topological transition.

\section{Current and Future Challenges}

By their definition, metamaterials represent an artificial media with "unnatural" but highly desirable electromagnetic properties, which are achieved through deliberate structuring on the subwavelength scale. However, in practice, limitations of fabrication techniques used for structuring always lead to the presence of variations in geometrical parameters over the extent of the metamaterial, which is equivalent to the presence of both disorder and local defects. In many practical applications such structural imperfection may substantially degrade performance of metamaterial based devices, and, for instance, lead to broadening of artificial resonances and undesirable back-reflection. In the latter case the effect of disorder can be circumvented by using the property of topological robustness. It was found that bianisotropy, also referred to as magneto-electric coupling, can play a role analogous to the spin-orbital coupling for electrons in solids. This is a key ingredient to Quantum Spin Hall Effect (QSHE) topological state, which now can be implemented for photons. The systems that exhibit OTT are usually metal dielectric structures 
where the dielectric constant along one or two of the directions can be made negative. In this context, one of the main issues is that of losses in metals. The recent efforts to develop non-metallic plasmonic materials such as transparent conducting oxides [9] is expected to partly address this issue. Another important challenge for metamaterials that undergo OTT is that of the severe phase mismatch between the high-k states in the metamaterials and free space. To alleviate this issue we have recently demonstrated the use of grating and photonic hypercrystal structures to efficiently out couple the high- $k$ states (Fig. 2b) [10]. The possibility of magnetization induced OTT allow one-way and nonreciprocal elements such as optical isolators and circulators. While there have been several reports of non-reciprocity in metamaterials relying on engineered resonances there by making them narrowband, the use of OTT presents a broadband approach which is crucial for wavelength multiplexing applications. Furthermore, the large PDOS in these systems also allow for miniaturization by enhancing naturally weak magnetooptical response.

\section{Advances in Science and Technology to Meet Challenges}

While some of early concepts of photonic topological metamaterials proposed were hard to implement due to the complexity of their design, recently, more practical designs have been proposed for microwave frequency range and topological robustness was unambiguously confirmed [7]. On the other hand, there is still no experimental demonstration available today for topological metamaterials at higher frequencies. This is due to the fact that early concepts used metal-based meta-atoms which become strongly absorptive at infrared and visible frequencies, thus diminishing advantages of topological protection. As for other branches of metamaterials, mitigating loss becomes the main challenge here. One of the possible solutions to this problem can be achieved with the use of the presently popular concept of all-dielectric metamaterials, where topological order should be reengineered based on magneto-electric response of dielectric meta-atoms. At the same time, to make its fabrication feasible with the use of conventional nanofabrication techniques, the design should be kept as simple as possible.

In the context of OTT and hyperbolic metamaterials, once again approaches to mitigate losses or identifying applications where one can afford some losses is important. For example one could envision the use of OTT and HMMs in high quantum efficiency LEDs where speed is desirable or in isolators where extinction is more important. Identifying suitable material systems that are compatible with each other from a process standpoint is necessary for the

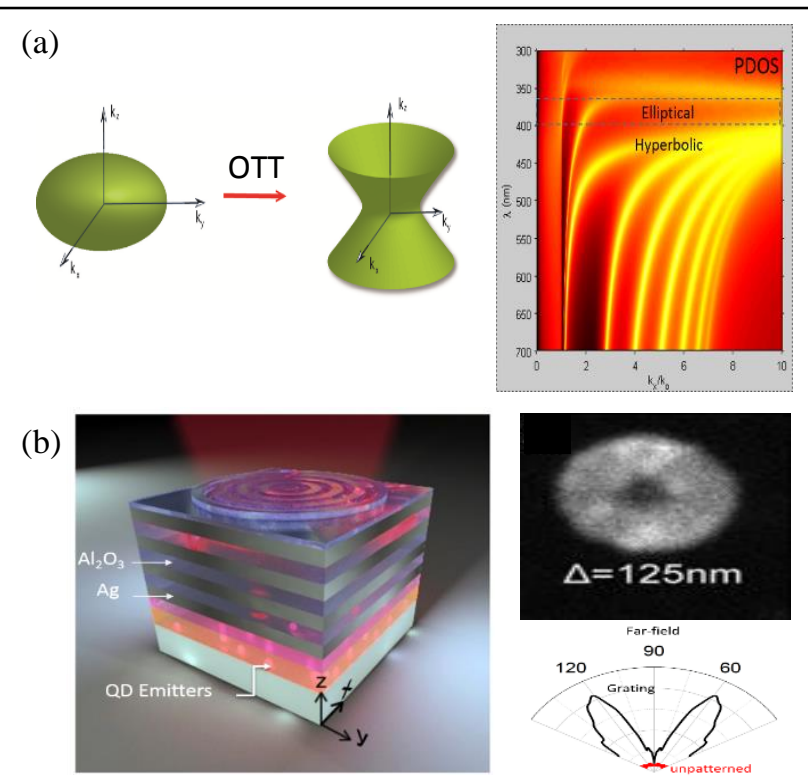

Fig. 2: (a) Optical topological transition in iso-frequency surface and the associated modification in photon density of states and (b) Bulls-eye grating used for outcoupling high-k states along with observed emission and far-field emission pattern.

magnetically tunable OTT. The advent of non-metallic plasmonic materials and new growth techniques such as atomic layer deposition provides avenues for exploring new applications and phenomena.

\section{Concluding Remarks}

In summary we discuss the use of concepts from the field of mathematical topology to realize two novel device concepts in photonics. The realization of topological protected transport in metamaterials provides an attractive approach to alleviate some of the outstanding issues in these systems such as disorder induced scattering. The OTT presents a new approach for broadband control of light-matter interaction and is expected to lead to practical device applications such as ultrafast LEDs and isolators.

\section{References}

[11] Hasan, M. Z. \& Kane, C. L., Rev. Mod. Phys. 82, 3045-3067 (2010).

[12] Haldane, F. \& Raghu, S., Phys. Rev. Lett. 100, 013904 (2008).

[13] Wang, Z., Chong, Y., Joannopoulos, J. D. \& Soljačić, M., Nature 461, 772-775 (2009).

[14] Khanikaev, A. B. et al., Nat. Mater. 12, 233-239 (2013).

[15] Hafezi, M., Mittal, S., Fan, J., Migdall, A. \& Taylor, J. M., Nat. Photon. 7, 1001-1005 (2013).

[16] Rechtsman, M. C. et al., Nature 496, 196-200 (2013).

[17] Chen, W.-J. et al., Nat. Commum. 5, 6782:1-7 (2014). 
[18] Krishnamoorthy, H. et al., Science 336, 205 (2012).

[19] Naik et al., Adv. Mat., 25, 3264 (2013)

[20] Galfsky et al., Optica 2, 62 (2015). 


\section{Functionally doped metal oxides for} future ultra-fast active metamaterials Marcello Ferrera, ${ }^{1,2}$ Nathaniel Kinsey, ${ }^{1}$ Clayton DeVault, ${ }^{1}$ Jongbum Kim, ${ }^{1}$ Vladimir Shalaev, ${ }^{1}$ and Alexandra Boltasseva ${ }^{1}$

${ }^{1}$ Purdue University

${ }^{2}$ Heriot-Watt University

\section{Status}

Plasmonics has brought many radically new device concepts into optical technologies. This is not only due to the possibility of overcoming the major limitations related to the diffractive nature of light, but also because introducing metal constituents (exhibiting electric resonances) into the world of photonics allows for developing extraordinary engineered composite materials with effective optical properties distributed over a more extensive region of the electromagnetic parameter space $(\varepsilon-\mu \text { space })^{1,2}$. However, after the initial impulse of noble metal-based optical metamaterials, including numerous successful proofof-concept experiments, a tremendous need for alternative, versatile, tunable and, CMOS-compatible materials is arising. In fact, in addition to overwhelming fabrication and integration challenges, gold and silver have a very limited tunability (here defined as the capability of changing the material's optical properties) and suffer from considerable losses.

Recently, significant attention has been given to alternative material platforms such as transition metal nitrides (TMNs) and transparent conductive oxides (TCOs), which overcome all the previously listed limitations intrinsic of gold and silver. For the case of TMNs (e.g. titanium and zirconium nitride - TiN, $\mathrm{ZrN}$ ), their optical properties resemble those of gold with a tailorable plasma frequency located in the visible/ultraviolet region. TMNs are robust, durable materials with exceptionally high thermal, chemical, and mechanical stability. They have been shown to provide plasmonic performance similar to that of gold structures and they are good candidates for heatassisted plasmonic applications such as thermo-photovoltaics, heat-assisted magnetic recording, and photocatalysis, due to their refractory nature (although this is not the focus of the present outlook) ${ }^{2,3}$. TCOs (e.g. aluminum-, gallium-, indium-doped zinc oxide [AZO, GZO, IZO], tin-doped indium oxide [ITO]), on the other hand, are highly doped wide band semiconductors with a plasma frequency typically located in the near infrared range. ${ }^{3}$ In the near-infrared, TCOs possess lower optical loss and a smaller magnitude of real permittivity $\left(\varepsilon^{\prime}\right)$ compared to noble metals and nitrides. Because of these characteristics,

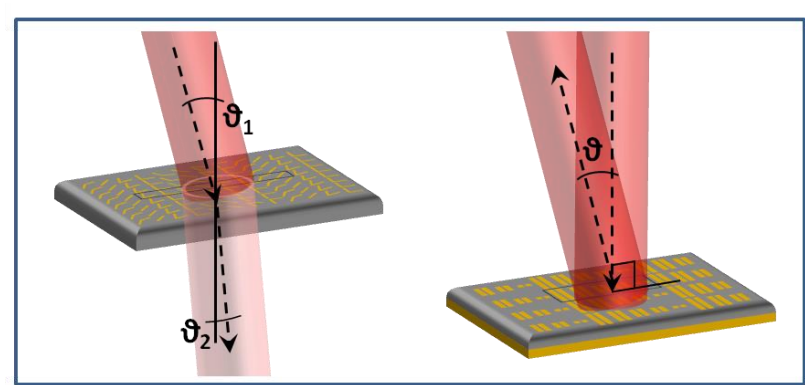

Figure 1 - Nanoantenna (left) and gap-plasmon (right) metasurfaces. The schematic depicts anomalous transmission and reflection plus the "efficiency Vs flexibility" trade-off typical of these structures.

TCOs allow one to access the Epsilon-Near-Zero (ENZ) regime in the near infrared for both composite structures and bulk materials ${ }^{4}$. In addition to this, complex oxides show a great dynamic tunability (here defined as the possibility to alter the dielectric permittivity by either optical or electric excitation). Consequently, all these features make TCOs exceptionally promising for a new generation of active metamaterials and plasmonic devices 5 .

\section{Current and Future Challenges}

During the last five years, the study and development of metamaterials has been largely focusing on the domain of metasurfaces. In contrast to complex fabrication of the proposed bulk metamaterial devices, two-dimensional metasurfaces are a technologically driven research area which can be fabricated using standard planar photolithographic processes, and their conceptual simplicity allows for a first approximation analysis using straightforward analytical tools.

Metasurfaces have proven to be effective for realizing numerous fundamental ultra-thin optical components such as lenses, polarizers, beamsteerers, holographic plates, etc ${ }^{6}$. Two fundamental approaches have been used for the design and realization of optical metasurfaces [see Fig.1]: i) nano-antennas (either made of metal or carved inside a metallic film ${ }^{6,7}$; ii) gapplasmons $^{8}$. The first strategy relies on controlling the phase of a propagating wavefront using subwavelength metallic nano-antennas. Following this method, numerous works on metasurfaces were developed after the pioneering work by the Capasso group on a generalized law of reflection and refraction. This technique has the advantage of being implemented using standard electron beam lithography or ion beam milling including only one processing step, but has a limited efficiency. In other words, only a moderate percentage of the incident light is influenced by the presence of the nano-patterned antennas. The gap plasmon solution, on the contrary, can maximize the desired optical efficiency at the cost of a multilayer 
structure, higher losses, and the impossibility of working in transmission.

Even though this technology is very promising, there are obstacles for the realization of impactful devices. First, we have to deal with the lack of CMOScompatibility with regards to gold and silver, which are still the most largely used metallic constituents for the fabrication of optical metasurfaces. Secondly, one should consider how to significantly enhance the performance of these devices in terms of power consumption, versatility, and real-time control over the optical properties.

\section{Advances in Science and Technology to Meet Challenges}

As previously stated, the first step towards the realization of "real-world" devices is related to the replacement of traditional noble metals (gold and silver) with CMOS-compatible materials. Here, a big step forward has been made by introducing TCOs to the realm of plasmonics. TCOs such as AZO and GZO can have significantly lower material losses $(\varepsilon ")$ than silver at telecommunication wavelengths with the additional benefit of being non-stoichiometric materials, thereby providing the capability of tailoring the intrinsic plasma frequency in a wide range from the visible to the near infrared (e.g. ITO 0.6-1.6 $\mu \mathrm{m}$ ). What makes these materials special is their optical transparency and their ability to sustain very high doping levels without effective degeneration of their structure. The achievable intrinsic carrier concentration $\left(\mathrm{N} \sim 10^{21} \mathrm{~cm}^{-3}\right)$ is very high compared to doped semiconductors (enabling metal-like properties in the NIR) and one order of magnitude smaller than noble metals $\left(\mathrm{N}_{\text {gold }}=5.9 \times 10^{22} \mathrm{~cm}^{-3}\right)$. Because of this moderately high concentration of free electrons, the absolute value of the real part of the dielectric permittivity is small, granting access to the ENZ regime $^{4}$ - a material science "sweet-spot" where the dynamic tunability of the optical properties is drastically enhanced together with the nonlinearities ${ }^{9}$. In addition, the fabrication technology underlining these materials is particularly mature thanks to their extensive use in industrial applications such as touchscreens, transparent electrodes on solar panels, and more recently in high-resolution flexible displays. For instance, high quality TCO thin films can be deposited by numerous techniques (e.g. sputtering, laser ablation, aqueous chemical deposition, liquid film spay deposition, etc.).

Consequently, TCOs represent a promising answer to the problems which plague noble-metal-based metamaterials - CMOS-compatibility, and tailorability together with dynamic tunability. However, two fundamental issues still remain to unlock the full

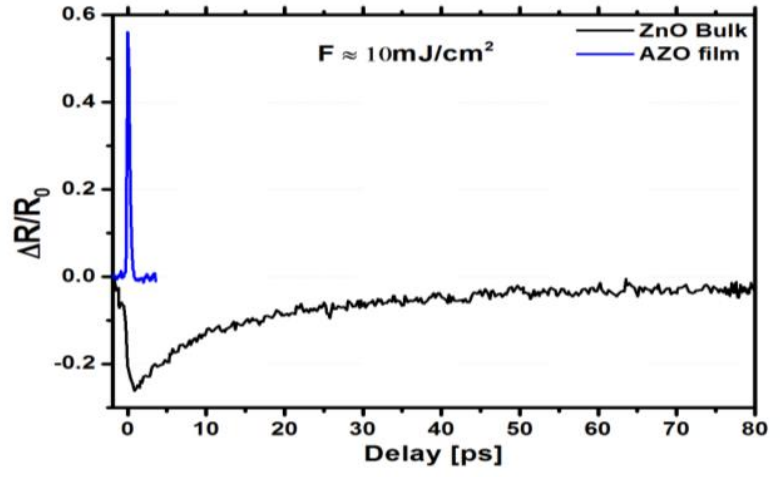

Figure 2 - Transient reflectance for commercially available mono-crystal $\mathrm{ZnO}$ substrates and $\mathrm{Al}: \mathrm{ZnO}$ thin films engineered to possesses ultrafast response and ENZ behavior in the NIR frequency range.

capability of these compounds in the realm of active metamaterials. The first problem deals with the limited absolute variation of the optically induced carrier concentration/permittivity (on the order of $10 \%$ of the intrinsic concentration for optical fluence levels well below the damage threshold) and the second issue is the slow electron-hole recombination time (up to 100s $\mathrm{ps})^{10}$. Solutions to these problems have to be found not only in the material science domain but also with respect to the optimization of existing designs and/or the realization of alternative schemes. With respect to the first concern, a limited modification of $\varepsilon$ can still enable large dynamic tuning when operating in the ENZ regime. In addition, gap plasmons could be used, which are inherently resonant and are thus more susceptible to the relative variation of $\varepsilon$. On the other hand, speeding up the recombination time is intrinsically a material problem. We have recently developed a novel room temperature fabrication process by which an ultrafast recombination time $(<1 \mathrm{ps})$ is attained in AZO films without paying the price of reduced optical properties [see Fig.2]. By optimizing the design/scheme of the metasurface as well as its material properties, both large dynamic tuning and ultrafast recombination can be achieved.

\section{Concluding Remarks}

In conclusion, it is our firm belief that the world of plasmonic and metamaterial devices has a bright future ahead as long as efforts in developing new materials remains high. With respect to the basic requirements that these novel compounds should possess, priority should be given to material platforms which are CMOS-compatible and whose optical properties can be engineered both statically and dynamically over a broad spectral range. In this direction, highly doped semiconductor oxides meet all the requirements, and appear to be key compounds for the realization of future tunable/active metamaterial devices. This is directly linked with TCO films engineered to exhibit 
an ultrafast optical response, low loss, and ENZ behavior over a wide wavelength range by controlling the material deposition. Due to this suite of desirable features, two directions that could experience a direct and immediate benefit from the development of such novel plasmonic compounds are the fabrication of flat meta-devices (metalenses, holographic plates, etc.) and switchable components (ultra-compact modulators, routers, switches, etc.). Therefore, we envision a fast and flourishing research avenue into TCO-based devices in the immediate future.

\section{References}

[1] A. Boltasseva, H. A. Atwater, "Low-Loss Plasmonic Metamaterials" Science, 331, 290-291 (2011).

[2] M. Wegener, "Metamaterials Beyond Optics" Science, 342, 939-940 (2013).

[3] U. Guler, A. Boltasseva, V. M. Shalaev, "Refractory plasmonics" Science, 344, 263-264 (2014).

[4] R. Maas, J. Parsons, N. Engheta, and A. Polman, "Experimental realization of an epsilon-near-zero metamaterial at visible wavelengths "Nat. photonics, 7, 907-912 (2013).

[5] V. J. Sorger, N. D. Lanzillotti-Kimura, R.-M. Ma, and X. Zhang, "Ultra-compact silicon nanophotonic modulator with broadband response" Nanophotonics 1, 17-22 (2012).

[6] N. Yu, F. Capasso,"Flat optics with designer metasurfaces" Nat. Materials, 13, 139-150 (2014).

[7] X. Ni, et al.,"Ultra-thin, planar, Babinet-inverted plasmonic metalenses" Light: Science and applications, 2, doi:10.1038/lsa.2013.28 (2013).

[8] A. Pors, O. Albrektsen, I. P. Radko, and S. I. Bozhevolnyi, "Gap plasmon-based metasurfaces for total control of reflected light" Scientific Reports 3, Article number: 2155 doi:10.1038/srep02155 (2013).

[9] M. Abb, Y. Wang, C.H. de Groot \& O. L. Muskens, "Hotspot-mediated ultrafast nonlinear control of multifrequency plasmonic nanoantennas" Nat. Commun. DOI: 10.1038/ncomms5869 (2014).

[10] D. B. Tice, et al.,"Ultra-fast modulation of the plasma frequency of vertically aligned indium tin oxide rods" Nano Lett. 14, 1120-1126, (2014). 
8. Optical dielectric metamaterials

and metasurfaces - Jason Valentine

Vanderbilt University

\section{Status}

The use of resonant dielectric particles as the unit cells of metamaterials was proposed at least as early as 1947 by Lewin [1]. Lewin realized that the first two Mie resonances of non-magnetic dielectric spheres correspond to electric and magnetic dipole resonances. As in the case of metallic dipole antennas and split ring resonators, these dielectric particles can be used to create metamaterials with extreme permittivities and permeabilities. However, unlike metallic metamaterials, all-dielectric metamaterials (ADMs) can exhibit extremely small absorption loss if they are operated at frequencies below the constituent material's bandgap. Furthermore, while metallic metamaterial unit cells are often complex, especially when a magnetic response is required, ADMs can be implemented with extremely simple geometries such as spheres and cubes (Fig. 1). This allows easier scalability of the material, more isotropic properties, and greatly aids in the fabrication of thicker threedimensional (3D) materials.

While dielectric metamaterials were first demonstrated at microwave frequencies, their development at infrared and visible frequencies has accelerated over the past several years. Work in these regimes began with the investigation of isolated magnetic resonances [2]-[4] in both individual particles and metamaterial arrangements. This work paved the way for more advanced implementations involving overlapped electric and magnetic resonances [5], [6]. Importantly, overlapping of the resonances can be achieved using only one structural element per unit cell, such as a disk or ellipsoid. This results in a fairly simple unit cell while still allowing access to complex optical properties. Work has also ventured into the investigation of Fano-resonant ADMs [7]-[9] with quality factors that far exceed their plasmonic counterparts. Progress has also included wavefront control using two-dimensional (2D) metasurfaces [10], [11] as well as the realization of 3D metamaterials [12]. Several of these advances are illustrated in Fig. 2.

\section{Current and Future Challenges}

One of the key challenges facing the dielectric metamaterial community is that the constituent dielectric must have a refractive index that is substantially larger than the surrounding index which is air in most cases. Since dielectric resonators act as cavities their size, and thus lattice period, is inversely proportional to the refractive index of the constituent material. As demonstrated by Sinclair et. al. [4],

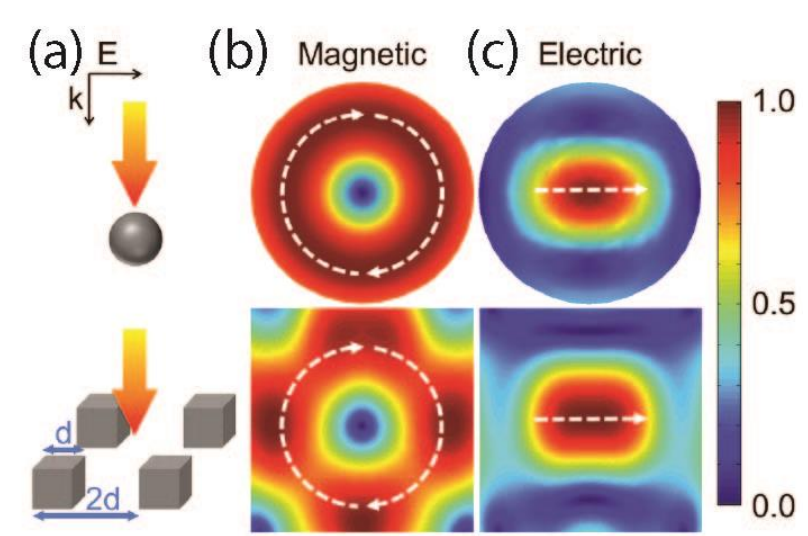

Figure 1. (a) Schematic of isolated spherical resonator and two-dimensional cube-based metamaterial.

Normalized electric field distributions for the (b) firstorder (magnetic) mode and (c) second-order (electric) mode. The white dashed lines and arrows indicate the field direction. Figure is reproduced from [4].

indices less than $n \sim 3.5$ will lead to significant spatial dispersion when both the first (electric) and second (magnetic) resonances are utilized (cubic resonators with a filling fraction of 50\%). While this should not be considered a hard limit, as it depends on the shape of the resonator and the filling fraction, it provides a good rule of thumb. The index requirement can be compared with photonic crystals (PCs). While PCs suffer from increased light leakage as the index contrast decreases, they are regularly implemented using much lower index materials such as silica glass $(n \sim 1.45)$, for instance in the case of optical fibers.

In the short to long-wavelength infrared regime smallbandgap semiconductors such as germanium $(n \sim 4)$, tellurium $(n \sim 5.5)$ and silicon $(n \sim 3.5)$ are viable options. For instance, in the telecommunications band silicon has been almost exclusively utilized to date. The real challenge lies in scaling dielectric metamaterials to visible frequencies due to the lack of high index and wide band-gap semiconductors. Choices are almost exclusively limited to III-V materials such as AlGaAs $(n \sim 3.8)$ and $\mathrm{GaP}(n \sim 3.4)$. However, these materials are considerably more difficult to grow than $\mathrm{Si}$ and etch chemistries are often not as widely known.

Since semiconductors are often used as the constituent material, active control of ADM optical properties is an attractive application. However, to date, this goal has not been achieved. One issue is that the relatively broad resonances of ADMs prevent index changes associated with thermo-optic and electro-optic effects from causing sufficient perturbations in the response. Narrow linewidth Fano-resonant ADMs may be a viable solution to this issue. A related area is the development of nonlinear ADMs where the nonlinear susceptibility of the resonator material is enhanced or 
(a)
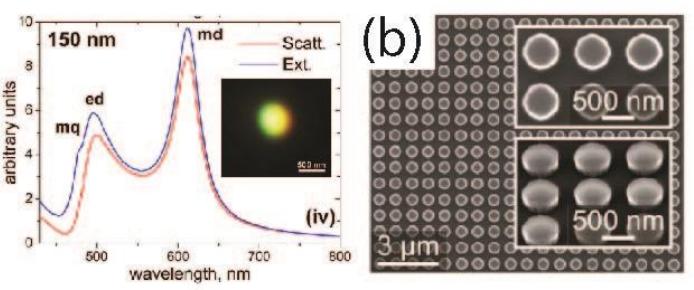

(c)
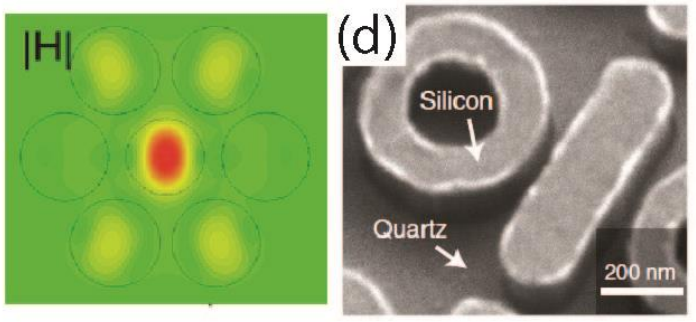

(e)

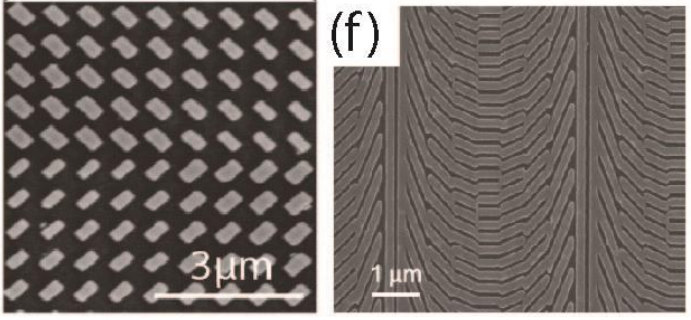

Figure 2. (a) Electric and magnetic modes in a Si sphere [2]. (b) Si nanodisk-based metamaterials [6]. (c) Fanoresonant Si oligomer [9] and (d) ring and bar-based metamaterial implementations [7]. (c,d) Metasurfaces for manipulating the phase of light [10], [11].

controlled using the unit cell geometry [13]. This area of research is also in its infancy but could be quite fruitful due to the excellent modal overlap that can be achieved with the resonator material.

In terms of passive ADMs, it is essential to reduce the cost of ADMs and thus scalable fabrication methods, not involving nanoscale lithography, must be developed. Due to the relatively simple unit cell geometry of ADMs, this route is potentially more straightforward than plasmonic variants. The use of colloidal particles, such as $\mathrm{Si}$ spheres, is a particularly attractive approach that is currently under development. However, techniques are also needed that allow more flexibility in controlling the unit cell geometry while allowing precise control over the underlying lattice and resonator filling fraction.

\section{Advances in Science and Technology to Meet Challenges}

While ADMs have recently been scaled to the visible by using $\mathrm{Si}$ [11], its absorption loss in this regime limits its usefulness. While III-IV materials such as AlGaAs have sufficient indices to realize visible ADMs, high quality thin films must be grown on lattice matched substrates. Unfortunately, these substrates invariably have a nearly identical index to the thin film. In order to realize an ADM this substrate must be removed to provide the large index contrast required to support a strong optical resonance in the unit cell. This is a considerable technical challenge due to the thin metamaterial layer thickness and small unit cell size, though it is certainly solvable. It should be noted that growth of an amorphous semiconductor on a low index substrate such as quartz is possible but many of the attractive optical and electronic properties of the semiconductor will be degraded. Another approach is the use of a lower index material such as $\mathrm{TiO}_{2}(n \sim 2.5)$ or a chalcogenide glass. However, more research is needed on the extent to which such a metamaterial could be homogenized.

There are several routes forward regarding the realization of active ADMs. In terms of tuning optical properties, one route is the use of high-quality factor resonances combined with a relatively weak electrooptical effect. Recent research has shown that high-Q resonances are possible in ADMs, however, the ultimate limit on the Q-factor is unclear and will depend on the fidelity with which the material can be fabricated as well as the absorption loss [7], [8]. Achieving broadband control is potentially more challenging as relatively large index changes within the unit cell must be achieved. Phase change materials such as $\mathrm{VO}_{2}$ and chalcogenide glasses provide a potential solution, though thermal recovery time may limit the speed of the device.

Controlling and enhancing light emission from ADMs is another area that has not been explored. While light sources such as quantum dots can be incorporated within ADMs it is potentially more interesting to use the resonator itself as the light source. To accomplish this, more work needs to be invested in forming ADMs from direct bandgap semiconductors or incorporating more complex architectures into the unit cell such as quantum wells. This is a particularly attractive area as a wide range of unit cell geometries and optical properties can be explored while preserving excellent modal overlap with the light emitting layer.

\section{Concluding Remarks}

The development of ADMs at infrared and visible frequencies is still in its infancy, even compared to the relatively young metamaterial field as a whole. However, the potential for this field is vast as many of the properties observed in plasmonic metamaterials are readily achievable in ADM variants with greatly reduced loss. Furthermore, the use of semiconductors as the constituent material provides a natural integration with active components and optoelectronic devices

\section{References}

[1] L. Lewin, "The electrical constants of a material loaded with spherical particles," Proc. Inst. Elec. Eng., Part 3, vol. 94, pp. 65-68, 1947. 
[2] A. I. Kuznetsov, A. E. Miroshnichenko, Y. H. Fu, J. Zhang, and B. Luk'yanchuk, "Magnetic light," Sci. Rep., vol. 2, p. 492, Jan. 2012.

[3] A. B. Evlyukhin, S. M. Novikov, U. Zywietz, R. L. Eriksen, C. Reinhardt, S. I. Bozhevolnyi, and B. N. Chichkov, "Demonstration of Magnetic Dipole Resonances of Dielectric Nanospheres in the Visible Region," Nano Lett., vol. 12, no. 7, pp. 3749-3755, Jul. 2012.

[4] J. C. Ginn, I. Brener, D. W. Peters, J. R. Wendt, J. O. Stevens, P. F. Hines, L. I. Basilio, L. K. Warne, J. F. Ihlefeld, P. G. Clem, and M. B. Sinclair, "Realizing Optical Magnetism from Dielectric Metamaterials," Phys. Rev. Lett., vol. 108, no. 9, p. 097402, Aug. 2012.

[5] Y. H. Fu, A. I. Kuznetsov, A. E. Miroshnichenko, Y. F. Yu, and B. Luk'yanchuk, "Directional visible light scattering by silicon nanoparticles.," Nat. Commun., vol. 4, p. 1527, Jan. 2013.

[6] I. Staude, A. E. Miroshnichenko, M. Decker, N. T. Fofang, S. Liu, E. Gonzales, J. Dominguez, T. S. Luk, D. N. Neshev, I. Brener, and Y. Kivshar, "Tailoring Directional Scattering through Magnetic and Electric Resonances in Subwavelength Silicon Nanodisks.," ACS Nano, vol. 7, no. 9, pp. 78247832, Aug. 2013.

[7] Y. Yang, I. Kravchenko, D. Briggs, and J. Valentine, "All-dielectric metasurface analogue of electromagnetically induced transparency," Nat. Commun., vol. 5, p. 5753, Dec. 2014.

[8] C. Wu, N. Arju, G. Kelp, J. a Fan, J. Dominguez, E. Gonzales, E. Tutuc, I. Brener, and G. Shvets, "Spectrally selective chiral silicon metasurfaces based on infrared Fano resonances.," Nat. Commun., vol. 5, no. May, p. 3892, 2014.

[9] A. E. Miroshnichenko and Y. S. Kivshar, "Fano resonances in all-dielectric oligomers.," Nano Lett., vol. 12, no. 12, pp. 6459-6463, Dec. 2012.

[10] Y. Yang, W. Wang, P. Moitra, I. I. Kravchenko, D. P. Briggs, and J. G. Valentine, "Dielectric MetaReflectarray for Broadband Linear Polarization Conversion and Optical Vortex Generation.," Nano Lett., vol. 14, no. 3, pp. 1394-1399, Feb. 2014.

[11] D. Lin, P. Fan, E. Hasman, and M. L. Brongersma, "Dielectric gradient metasurface optical elements," Science (80-. )., vol. 345, no. 6194, pp. 298-302, Jul. 2014.

[12] P. Moitra, Y. Yang, Z. Anderson, I. I. Kravchenko, D. P. Briggs, and J. Valentine, "Realization of an all-dielectric zero-index optical metamaterial," Nat. Photonics, vol. 7, pp. 791-795, Jul. 2013.

[13] M. R. Shcherbakov, D. N. Neshev, B. Hopkins, A. S. Shorokhov, I. Staude, E. V. Melik-Gaykazyan, M.
Decker, A. A. Ezhov, A. E. Miroshnichenko, I. Brener, A. A. Fedyanin, Y. S. Kivshar, " Enhanced third-harmonic generation in silicon nanoparticles driven by magnetic response," Nano Lett., vol. 14, no. 11, pp. 6488-6492, Oct. 2014. 


\section{Beam shaping with metasurfaces - Carl}

Pfeiffer and Anthony Grbic

University of Michigan

\section{Status}

Controlling the polarization, phase, and amplitude of light is critical to numerous optical systems. Polarization control is typically achieved with combinations of linear polarizers and waveplates, while phase control is achieved using dielectric lenses and spatial light modulators. However, these conventional systems are bulky and do not lend themselves to nanophotonic system integration. The development of optically-thin metasurfaces promises to significantly reduce the size of optical systems. Metasurfaces are the two-dimensional equivalent of metamaterials, and are lower profile, lower loss, and simpler to fabricate than their three-dimensional counterparts. One can envision replacing bulky optical setups requiring combinations of conventional components with ultra-thin metasurfaces. Such advances will pave the way for flat/low profile, integrated optical devices.

To date, metasurfaces exhibiting a purely electric response have provided extraordinary capabilities in controlling electromagnetic wave fronts. Some of the most exciting examples have been subwavelength focusing and the generalization of Snell's laws of refraction [1]. More recently, it was shown that adding a magnetic response to metasurfaces can remove reflection losses and dramatically increase their transmission efficiency [2]. Anisotropy and magnetoelectric coupling were also systematically incorporated into metasurface design to allow for complete control of an electromagnetic wave front's polarization and phase [3]. Thus metasurfaces are able to transform an arbitrary incident optical wave front into any desired transmitted wave front. For example, metasurfaces have been designed to convert circularlypolarized Gaussian beams into radially polarized Bessel beams [4] (see Fig. 1). In addition, they have the potential to offer improved performance over conventional dielectric lenses since they can be designed to eliminate spherical and chromatic aberrations [5].

\section{Current and Future Challenges}

Although metasurfaces show great promise for dramatically reducing the size and complexity of optical systems, metasurfaces are typically too inefficient for many applications. Generally, metasurfaces have higher reflection and absorption losses than conventional optical components. The high absorption also restricts their maximum power handling capability [6]. In addition, metasurfaces

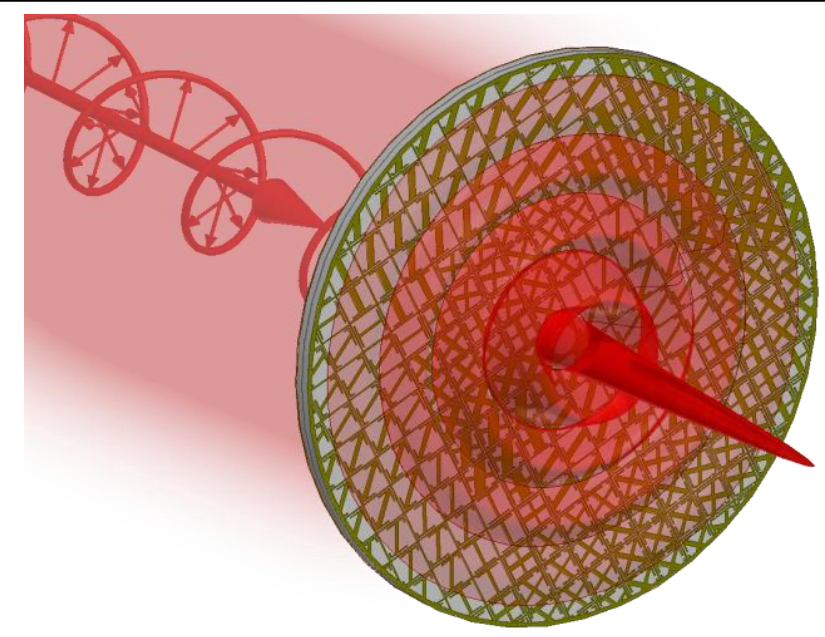

Figure 1 - Metasurfaces have demonstrated the ability to transform an arbitrary incident wave front into any desired wave front. For example, an inhomogeneous, anisotropic metasurface transforms a circularly polarized Gaussian beam into a radially-polarized Bessel beam.

typically have stronger aberrations than conventional optical components.

The spatial light modulator is a centerpiece reconfigurable component for optical systems since it can provide complete phase and amplitude control. Developing ultra-thin metasurfaces that achieve a similar functionality to the spatial light modulator would be extremely attractive. Tunable metasurfaces promise to provide higher speeds, polarization manipulation, and smaller pixel sizes than spatial light modulators for more extreme control over optical wavefronts. However, the vast majority of optical metasurfaces are not tunable. And those that are, have limited tuning ranges $[7,8]$.

Although metasurfaces are substantially easier to fabricate than bulk metamaterials, challenges in fabrication and experimental validation of simulated results remain a significant issue. To achieve nanometer resolution, metasurfaces are usually fabricated using electron beam lithography or focused ion beam etching, which are expensive and time consuming processes. In addition, it is difficult to accurately pattern large areas using these technologies. Experimentally validating a metasurface design at optical frequencies requires extensive nanofabrication expertise. Therefore, many researchers develop, fabricate, and measure metasurfaces at microwave and millimeter-wave frequencies to demonstrate a proof-ofconcept [4]. However, this can provide limited insight into the performance of optical metasurfaces.

Advances in Science and Technology to Meet Challenges 
Several different technologies need to be advanced in order to address the current limitations of metasurfaces for beam shaping. Improving the efficiency of metasurfaces will require lower loss materials. Oxides and nitrides are promising alternatives to $\mathrm{Au}$ and $\mathrm{Ag}$, since their plasma frequencies can be controlled, which could provide lower loss depending upon the application [9]. Alternatively, patterning high index dielectrics such as $\mathrm{Si}$ shows significant potential since these materials have extremely low loss [10]. However, dielectric based metasurfaces have yet to achieve the same degree of polarization control that plasmonic metasurfaces offer.

Reconfigurable metasurfaces have the potential to provide arbitrary optical transfer functions on demand. For example, we can envision a future smartphone camera consisting of reconfigurable metasurfaces that are capable of providing high numerical aperture lenses for diffraction limited microscopy one instant and longer-focus lenses for imaging distant objects the next (see Fig. 2). Adding tunability to metasurfaces could also improve their performance. The beam generated by the metasurface could be monitored by a sensor, and this information fed back to tune the metasurface to reduce aberrations and improve the overall performance. New materials and designs are required to achieve this level of reconfigurability. One possible route involves using materials with an electronically controllable permittivity/sheet conductance such as liquid crystals and graphene, respectively [7]. Alternatively, the physical geometry of the metasurface can be controlled by applying DC currents or voltages across the structure [8]. Further, systematic design procedures need to be developed so that the metasurface's properties can be precisely controlled.

A significant impediment towards the advancement of metasurface technologies is the large cost and time required to develop prototypes. Metasurface designs that use standard materials are reasonably well predicted by full-wave simulations. However, the goal is to integrate new materials into metasurfaces to reduce losses and add tunablility. This work will require considerable experimentation to develop accurate models for characterizing the properties of these novel materials. This process will be expedited by more research groups having access to the development of prototypes. Advances in nanofabrication processes could certainly help reduce cost and improve accuracy. Alternatively, developing CMOS compatible optical materials would allow optical metamaterials to be fabricated alongside integrated circuits [9]. Thus optical metasurfaces could leverage the many years of research and development invested into the semiconductor industry.

\section{Concluding Remarks}

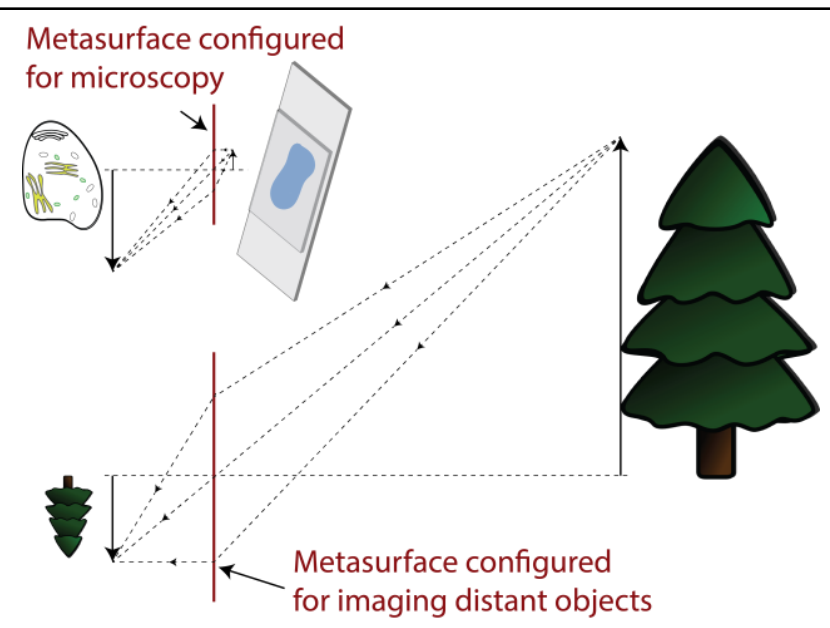

Figure 2 - Reconfigurable metasurfaces could provide a wide range of optical transfer functions. For example, a tunable metasurface could be reconfigured to focus on objects that are extremely close or far from an image sensor for microscopic and telescopic applications, respectively.

Optical metasurfaces offer unprecedented opportunities for tailoring optical wave fronts at will. They promise to offer a compact alternative to conventional optical components. Although promising, their performance, tunability, and cost must be addressed before they become ubiquitous optical elements. If any one of these issues sees significant progress in the upcoming years, metasurfaces could become standard components in optical systems.

\section{References}

[1] Yu N, Genevet P, Kats M A, Aieta F, Tetienne J P, Capasso F, Gaburro Z 2011 Light propagation with phase discontinuities: generalized laws of reflection and refraction Science 334333

[2] Pfeiffer C, Grbic A 2013 Metamaterial Huygens' surfaces: tailoring wave fronts with reflectionless sheets Phys. Rev. Lett. 110197401

[3] Pfeiffer C, Zhang C, Guo L J, Grbic A 2014 High performance bianisotropic metasurfaces: asymmetric transmission of light Phys. Rev. Lett. 113023902

[4] Pfeiffer C, Grbic A 2014 Controlling vector Bessel beams with metasurfaces Phys. Rev. Applied 2 044012

[5] Aieta F, Kats M A, Genevet P, Capasso F 2015 Multiwavelength achromatic metasurfaces by dispersive phase compensation Science 3471342

[6] Guler U, Boltasseva A, Shalaev V M 2014 Refractory plasmonics Science $\mathbf{3 4 4} 263$

[7] Chen H T, et al. 2008 Experimental demonstration of frequency-agile terahertz metamaterials Nature Photonics 2295

[8] Valente J, Ou J Y, Plum E, Youngs I J, Zheludev N I 2015 Reconfiguring photonic metamaterials with 
4

5

6

7

8

9

10

11

12

13

14

15

16

17

18

19

20

21

22

23

24

25

26

27

28

29

30

31

32

33

34

35

36

37

38

39

40

41

42

43

44

45

46

47

48

49

50

51

52

53

54

55

56

57

58

59

60

currents and magnetic fields Appl. Phys. Lett., 106 111905

[9] Ferrera M, Kinsey N, DeVault C, Kim J, Shalaev V, Boltasseva A 2016 Functionally doped metal oxides for future ultra-fast active metamaterials Journal of Optics VOL\# PAGE\#

[10] Valentine J 2016 Optical dielectric metamaterials and metasurfaces Journal of Optics VOL\# PAGE\# 


\section{Contol of emission and absorption with} metamaterials - Evgenii Narimanov

Purdue University

\section{Status}

Electromagnetic absorption holds the key for taking a new optical technology to the realm of practical applications - from the first anti-reflective coating discovered by Lord Rayleigh in 1879 [1] to optimized narrow-band emitters for thermal photovoltaics [2] to the ultra-low loss silica glass that lead to the fiberoptical communication systems [3] which brought us the world of internet connectivity. With the wavelength-specific absorption and emission connected to each other by the Kirchoff law [4], the control of electromagnetic absorption becomes an essential element of a new optical material technology.

With the choice of conventional optical materials generally limited by practical considerations, the standard approach to controlling electromagnetic absorption generally relies on either introducing resonant elements when particular response is needed for an a priori known frequency, or using impedancematched layers when broadband performance is desired. The resulting fundamental constrains to allow for optical interference in the former approach or adiabatic evolution in the latter, lead to the lower bound for the dimensions of the corresponding structures on the order of the corresponding wavelength. As it generally exceeds the size of a modern electronic transistor by nearly two orders of magnitude, to make the emerging optoelectronic technology compatible with modern integrated circuits, we need an entirely new and different approach to control the optical absorption.

The solution to this conundrum of the scale incompatibility can be found in the field of optical metamaterials. These composites offer the control of electromagnetic material response based on spatial patterning on a deeply subwavelength scale, thus offering the freedom from the size limits of conventional optical technology. Furthermore, even a "meta-surface" - the system that consists of only one or few "meta-atomic" layers of such a composite - has a strong effect on the optical absorption and reflection [5].

Starting from the first demonstration of metamaterial absorber for microwave range in 2008 [6], resonant metamaterials and metasurfaces have now been demonstrated for nearly the entire electromagnetic range - from visible and IR down to radio frequencies, [7] with the total thickness as low as $\lambda / 79$ [8]. Furthermore, the metamaterial technology has now been adapted to the design and fabrication of resonant

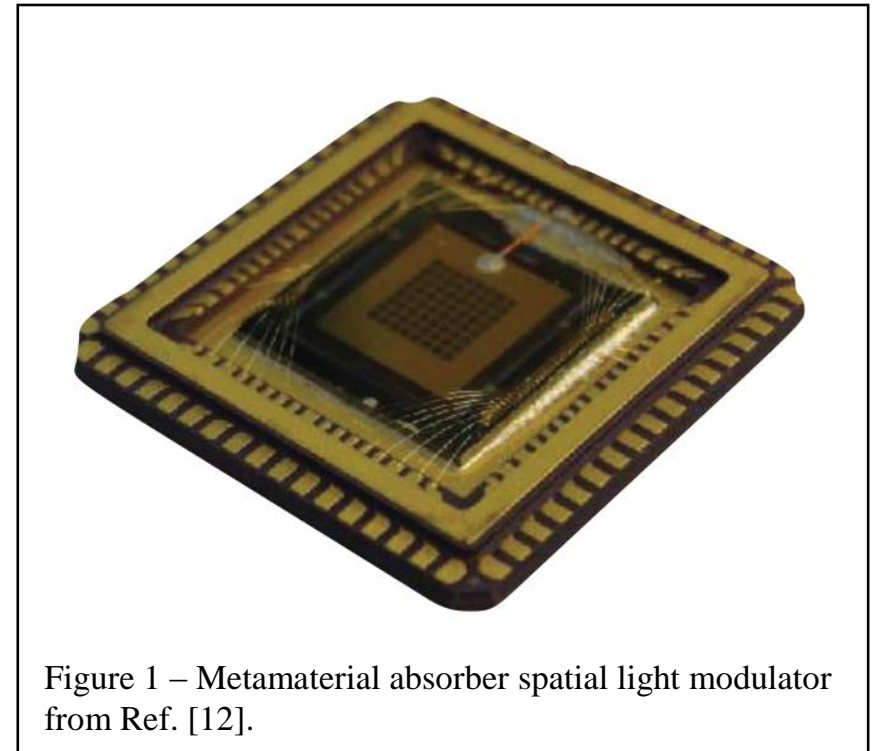

thermal emitters [9] for thermal photovoltaic applications.

While the desired broadband response can be achieved by combining different resonant elements within the same metamaterial [7], taking advantage of the broadband super-singularity of the photonic density of states in hyperbolic metamaterials [10] allows for a more "elegant" solution, with an important added benefit of relatively high degree of tolerance to material damage and disorder [11].

\section{Current and Future Challenges}

Metamaterials with designed electromagnetic absorption and emission also offer the capability of dynamic control for the response of the device, which has many advantages for spectroscopic and imaging applications. However, while such devices have been fabricated for $\mathrm{THz}$ frequency range [12], extending their performance to the optical domain, which implies increasingly more stringent requirements on the dimensions of the unit cell and material imperfections, remains a major challenge.

Another, and perhaps even more important, capability that may be offered by the metamaterial approach to controlled emission and absorption, is the potential to bridge the gap between the nanoscale quantum emitters (such as e.g. single atoms, organic dye molecules, or quantum dots) and micron-scale optical wavelength. In the near field of a metamaterial, such an emitter can "resolve" the subwavelength details of the metamaterial unit cell, which allows the use of its geometry to optimize the resulting light-matter interactions. As a result, these "quantum metamaterials" may hold the key to a practical "onchip" implementation of quantum optical communications and computations - but more work, 
both theoretical and experimental, is needed to reach this objective.

However, material losses inherent to all metamaterials that contain metallic and polaritonic media, will drastically limit the resulting coherence and entanglement range in such systems. Despite recent progress in the optimization of metamaterial design, the control of material loss in these composites remains a major challenge.

\section{Advances in Science and Technology to Meet Challenges}

Recent development of photonic hypercrystals [13], the hyperbolic metamaterials with periodic spatial variation of dielectric permittivity on subwavelength scale, that combine the features of optical metamaterials and photonic crystals, may help to address the challenge of coupling to quantum emitters. Even the simplest realization of the hypercrystal by the fabrication of high optical contrast subwavelength grating on top of a hyperbolic metamaterial (see Fig. 2), allowed for both enhanced spontaneous emission from quantum dots incorporated into the system, and efficient light extraction into the free space [14]. Although the actual fabrication of a hypercrystal with its inherent multi-scale structure is highly nontrivial, it can be accomplished using the standard methods that have already been used in nanophotonics.

While solving the problem of material loss in plasmonic composites is more challenging, it has been recently demonstrated that it can be addressed with proper electronic bandgap engineering [16]. However, while the fundamental theoretical possibility of the "lossless metal" was demonstrated in Ref. [16], it is yet unclear how to actually fabricate this elusive compound. Solving this problem is probably the primary challenge for the entire metamaterial community.

\section{Concluding Remarks}

With remarkable recent progress in the field of $\mathrm{THz}$ and optical metamaterials, the built-to-order metamaterial emitters and absorbers are entering the realm of practical devices and applications. Furthermore, it is likely that quantum metamaterial systems will become the key elements in future quantum information systems.

\section{References}

[1] Rayleigh, L. On reflection of vibrations at the confines of two media between which the

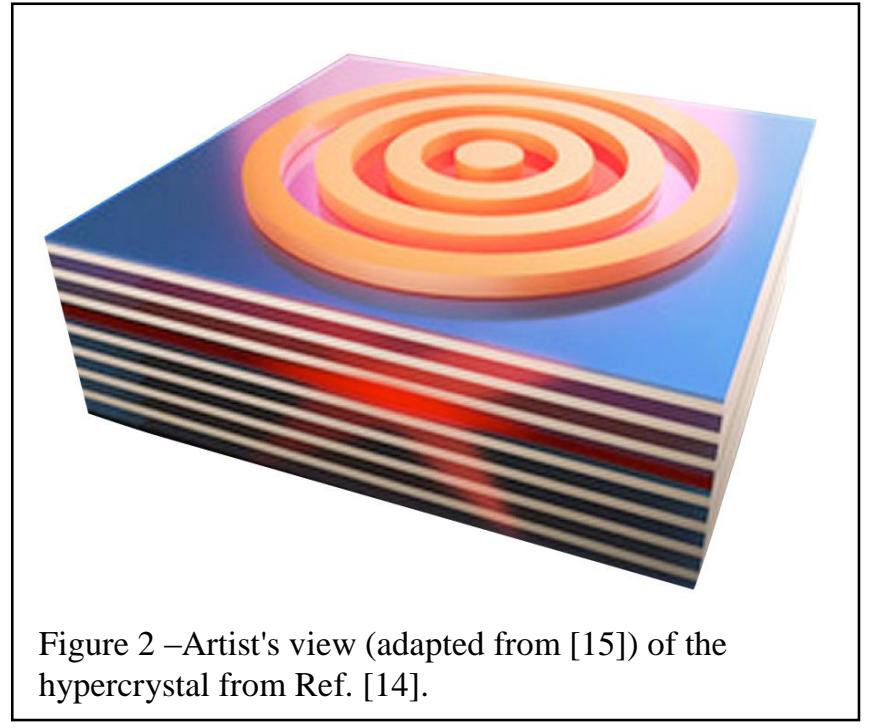

transition is gradual. Proceedings of the London Mathematical Society s1-11, 51 (1879).

[2] I. Celanovic, P. Bermel, and M. Soljacic, Thermophotovoltaic power conversion systems: current performance and future potential Oyo Buturi (Japan Society of Applied Physics) 80 (8), 687 (2011).

[3] J. M. Senior and M. Y. Jamro, Optical fiber communications: principles and practice. Pearson Education, 2009.

[4] G. Kirchhoff, Ueber das Verhältniss zwischen dem Emissionsvermögen und dem Absorptionsvermögen der Körper für Wärme and Licht. Annalen der Physik und Chemie 109 (2), 275 (1860).

[5] N. Yu and F. Capasso, Flat optics with designer metasurfaces. Nature Materials 13, 139 (2014).

[6] N. I. Landy, S. Sajuyigbe, J. J. Mock, D. R. Smith, W. J. Padilla, Physical Review Letters. 100, 207402 (2008).

[7] C. M. Watts, X. Liu, W. J. Padilla, Metamaterial Electromagnetic Wave Absorbers. Advanced Materials 24, OP98 (2012).

[8] Y. Cheng, H. Yang, Z. Cheng, N. Wu, Perfect metamaterial absorber based on a split-ring-cross resonator. Applied Physics A 102, 99 (2011).

[9] I. Puscasu, W. L. Schaich, Narrow-band, tunable infrared emission from arrays of microstrip patches. Applied Physics Letters 92, 233102 (2008).

[10] I. I. Smolyaninov and E. E. Narimanov, Metric Signature Transitions in Optical Metamaterials. Physical Review Letters 105, 067402 (2010)

[11] E. E. Narimanov, H. Li, Yu. A. Barnakov, T. U. Tumkur, and M. A. Noginov, Reduced reflection from roughened hyperbolic metamaterial. Optics Express 21 (12), 14956 (2013)

[12] D. Shrekenhamer, J. Montoya, S. Krishna, and W. J. Padilla, Four-Color Metamaterial Absorber 
THz Spatial Light Modulator. Advanced Optical Materians 1, 905 (2013).

[13] E. E. Narimanov. Photonic Hypercrystals, Physical Review X 4, 041014 (2014)

[14] T. Galfsky, H. N. S. Krishnamoorthy, W. Newman, E. E. Narimanov, Z. Jacob, and V. M. Menon, Active hyperbolic metamaterials: enhanced spontaneous emission and light extraction. Optica 2 (1), 62 (2015)

[15] S. Wills, A Bullseye for Brighter Metamaterials. Optics and Photonics News, January 2015

[16] J. B. Khurgin and G. Sun, In search of the elusive lossless metal. Applied Physics Letters 96, 181102 (2010) 


\section{Control of far-field thermal emission properties through the use of photonic structures - Linxiao Zhu and Shanhui Fan Stanford University}

\section{Status}

Absorption and thermal emission lie at the center of many energy conversion processes. The advent of photonic structures such as meta-materials and photonic crystal provides new capabilities for controlling emission and absorption properties. In recent years, significant efforts have been devoted to the use of various kinds of meta-material photonic structures, including photonic crystals [1-4], multilayer films [5,6], metal-insulator-metal structure [7], epsilonnear-zero (ENZ) and epsilon-near-pole (ENP) metamaterial [8], for the control of thermal emission and absorption properties. These structures enable thermal emission properties that are drastically different from conventional structures. For example, while thermal emission from conventional structures is typically thought of as spectrally broad and spatially incoherent, the use of photonic structures can lead to thermal emission that is spectrally selective, or spatially coherent [1]. These novel capabilities of controlling thermal emission, in turn have led to new potential application opportunities such as in thermophotovoltaics $[3,5,8]$ and radiative cooling $[6,9,10]$, where the capability for such spatial and spectral control of thermal emission is essential for enhanced performance in these applications.

\section{Current and Future Challenges}

The development of thermal emitters based on metamaterials and photonic structures, as well as the new applications such emitters have led to, in turn have challenged us to re-examine some of the fundamental constraints that were commonly thought to be intrinsic to thermal emitters, and to develop new capabilities to remove some of these constraints. Here, we highlight two important challenges:

(1) The design of thermal emitters with total emission power exceeding the apparent blackbody limit. In the applications for conversion of thermal radiation to work, it is desirable to generate as much thermal emission power as possible from a given thermal emitter. Therefore, it is important to re-examine the blackbody limit that has thought to be the fundamental limit that governs the emission from a given macroscopic thermal emitter to the far field.

(2) Most textbooks on thermal emission state the Kirchhoff's law of detailed balance, i.e. the spectral and angular absorptivity and emissivity being equal, as a fundamental law for thermal emission. On the other hand, it is important to emphasize that the Kirchhoff's
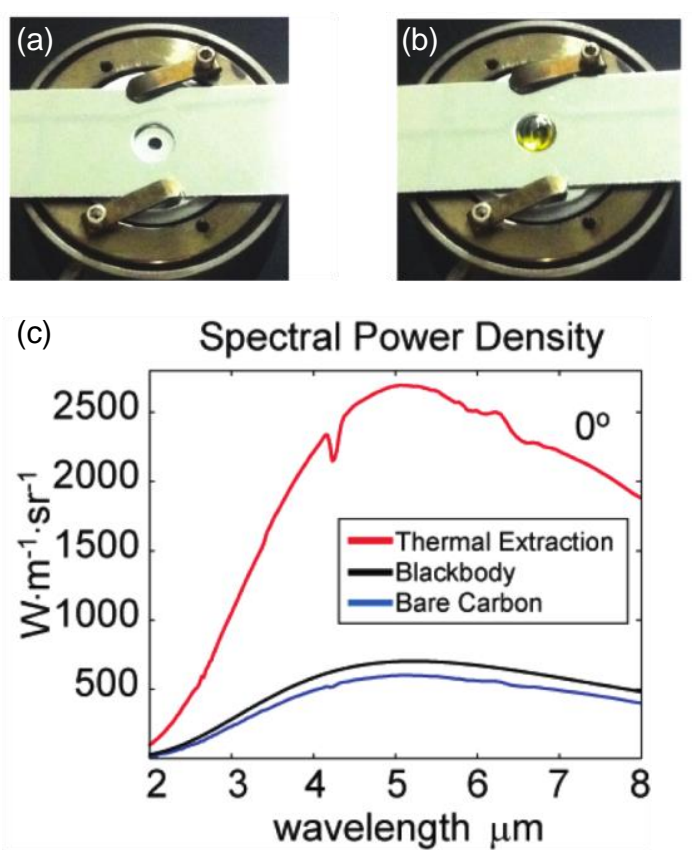

FIG. 1. (a) Emission source made of carbon dot is coated on an $\mathrm{Al}$ plate placed on a temperature-controlled heater. (b) Thermal extraction device made of $\mathrm{ZnSe}$ hemisphere is placed in close contact with carbon dot. (c) Emission power spectra measured at $553 \mathrm{~K}$ at normal direction. The blue and red curves correspond to the source in (a) and (b), respectively. The black curve is the emission from a blackbody. Reprinted from Ref. [Error! Bookmark not defined.].

law is not a consequence of the second law of thermodynamics, but rather arises because most of the materials are reciprocal in their dielectric response. Also, from a perspective of thermal and solar energy harvesting, being able to violate detailed balance is in fact of fundamental importance in order to reach the highest conversion efficiency that the second law of thermodynamics allows for. A key challenge then is to develop non-reciprocal photonic structures that violate detailed balance.

\section{Advances in Science and Technology to Meet Challenges}

Here we highlight two recent advances that should motivate us to carefully re-examine some of the common notions associated with thermal emission.

(1) The development of thermal extraction scheme. A thermal extraction scheme for enhancing the thermal emission of a given emitter beyond the apparent blackbody limit has been theoretically proposed and experimentally demonstrated in Ref. [11]. In the thermal extraction scheme, as shown in Figure 1, one places a thermal emitter in the electromagnetic thermal near field of a thermal extraction medium. In the experimental setup shown in Figure 1, the thermal emitter consists of a layer of carbon black placed on an aluminum substrate, and the thermal extraction medium is a $\mathrm{ZnSe}$ hemispherical lens. The thermal emitter has a refractive index that is higher than 
vacuum, consequently, within the thermal emitter there is a higher thermal electromagnetic energy flux as compared to vacuum at the same temperature. In the conventional setup, where the emitter is in direct contact to far field vacuum, this additional electromagnetic energy flux cannot escape the emitter. On the other hand, the use of the extraction medium, which has an index higher the vacuum, allows such thermal energy flux inside the emitter to be extracted into the extraction medium first. If the area of the extraction medium is significantly larger than that of the emitter, the extracted energy flux can then be emitted to the far field vacuum. The experiment shown in Figure 1 indeed demonstrates thermal emission that is significantly beyond the blackbody limit. An interesting emerging direction moreover may utilize meta-materials as the extraction mechanism. In Ref. [12] a hemisphere consisting of hyperbolic metamaterial is proposed to further enhance the thermal emission power for thermal extraction.

(2) The development of thermal emitters that maximally violate detailed balance. An advance is made in Ref. [13] in achieving near-complete violation of detailed balance, i.e. to achieve near unity contrast between spectral angular emissivity and absorptivity, at the same angle and wavelength. As shown in Figure $2 \mathrm{a}$, the non-reciprocal thermal emitter consists of a magneto-optical photonic crystal, made of n-InAs, atop a perfect electric conductor (PEC) reflector. By applying a magnetic field in the transverse direction, the contrast between absorptivity and emissivity at the same direction can be as large as $12.7 \mathrm{~dB}$, at a wavelength of $15.92 \mu \mathrm{m}$ (Figure 2b). Such capability of near-completely violating the detailed balance opens up new possibility for enhancing energy efficiencies, including solar energy harvesting and thermal radiation energy conversion. Also, from a theoretical point of view, for non-reciprocal emitters, the approach of inferring the emission properties from absorption will no longer be applicable, and a direct calculation of thermal emission properties using the formalism of fluctuational electrodynamics becomes essential. Nonreciprocal thermal emitter represents an important direction for emission and absorption control.

\section{Concluding Remarks}

In summary here, photonic structures such as metamaterials and photonic crystals, can be utilized to generate properties of thermal radiation that are drastically different from conventional thermal emitters. Significant efforts in the past decade have focused on control of spectral, angular and coherent properties of thermal emission from photonic structures. On the other hand, the use photonic structures should also enable us to re-examine some of the perceived fundamental constraints on the thermal emission, such as the blackbody limit, and the detailed

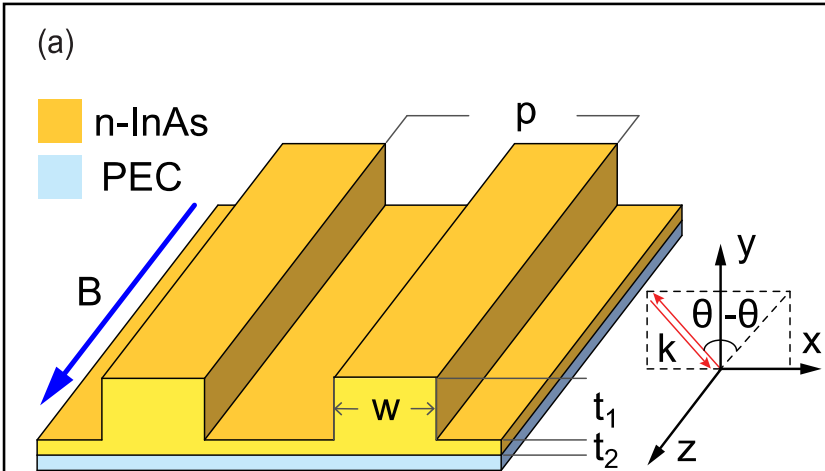

(b)

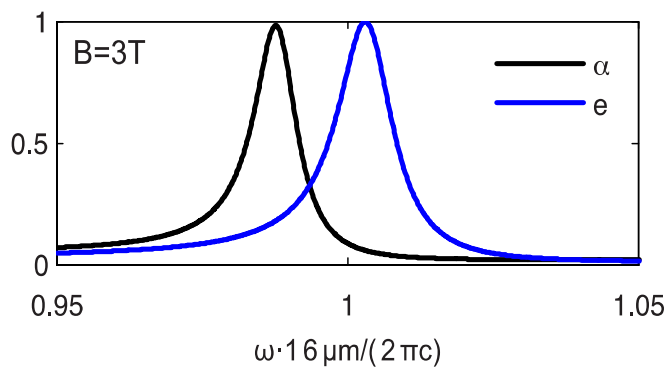

FIG. 2. (a) A schematic of a photonic crystal structure for maximal violation of detailed balance. The structure is periodic in the $\mathrm{x}$ direction, and has the following parameters: $\mathrm{p}=7.24 \mu \mathrm{m}, \mathrm{w}=3.2 \mu \mathrm{m}, \mathrm{t}_{1}=1.981 \mu \mathrm{m}$, and $\mathrm{t}_{2}=0.485 \mu \mathrm{m}$. An external magnetic field is applied in the $\mathrm{z}$ direction. TM polarization with an electric field in the $x-y$ plane is considered. (b) Absorptivity $(\alpha)$ and emissivity spectra at $\theta=61.28^{\circ}$ and $B=3 \mathrm{~T}$. Reprinted from Ref. [13].

balance. We anticipate that the use of meta-material can lead to thermal emitters that are fundamentally different from conventional thermal emitters, and open new application possibilities in numerous application areas such as energy conversion, imaging and sensing.

\section{References}

[1] Greffet J, Carminati R, Joulain K, Mulet J, Mainguy S and Chen Y 2002 Nature 416, 61

[2] Zoysa M, Asano T, Mochizuki K, Oskooi A, Inoue T and Noda S 2012 Nat. Photonics 6, 535

[3] Arpin K et al. 2013 Nat. Commun. 4, 2630

[4] Inoue T, Zoysa M, Asano T and Noda S 2014 Nat.

Mater. 13, 928

[5] Lenert A, Bierman D, Nam Y, Chan W, Celanovic I, Soljacic M and Wang E 2014 Nat. Nanotechnol. 9, 126 [6] Raman A, Anoma M, Zhu L, Rephaeli E and Fan S 2014 Nature 515, 540

[7] Liu X, Tyler T, Starr T, Starr A, Jokerst N and Padilla W 2011 Phys. Rev. Lett. 107, 045901

[8] Molesky S, Dewalt C and Jacob Z 2013 Opt. Express 21, A96

[9] Zhu L, Raman A and Fan S 2015 Proc. Natl. Acad. Sci. U.S.A. 112, 12282

[10] Hossain M, Jia B and Gu M 2015 Adv. Opt. Mater. 3, 1047 
[11] Yu Z, Sergeant N, Skauli T, Zhang G, Wang H and Fan S 2013 Nat. Commun. 4, 1730

[12] Simovski C, Maslovski S, Nefedov I, Kosulnikov S,

Belov P and Tretyakov S 2015 Photonic Nanostruct 13, 31

[13] Zhu L and Fan S 2014 Phys. Rev. B 90, 220301(R) 


\section{Engineering mid-Infrared and optical nonlinearities with metamaterials - Andrea Alù}

\author{
The University of Texas at Austin
}

\section{Status}

Nonlinear effects in optical materials are generally weak, yet they are of fundamental importance for frequency generation, optical sources, laser diagnostics, optical switching and modulation, integrated nanophotonic systems, and much more. Due to the typically small extent of optical nonlinearities, large light intensities and long propagation distances are required to produce detectable nonlinear responses. Phase matching between pump and generated signals, as they propagate in the nonlinear material, becomes crucial, making nonlinear optical systems convoluted and not always practical.

Metamaterials and metasurfaces, respectively 3D and 2D artificial materials with unusual electromagnetic responses, have been largely explored in recent years to go beyond these limitations [1-2]. Large field enhancements supported by the plasmonic constituents of metamaterials have been exploited to enhance optical nonlinearities. The unusual propagation properties of $\varepsilon$-near-zero metamaterials have been shown to be well suited to relax phase matching constraints and enhance nonlinear responses [3-4]. Still, the extent to which large nonlinear responses can be achieved in small volumes with conventional material substrates appears to be fundamentally limited.

In order to enhance their optical nonlinear response, material substrates can be engineered at the quantum level to support very large nonlinear responses, significantly larger than natural nonlinear crystals [5]. Very large nonlinearities can be obtained around intersubband transitions in semiconductors [6-7], and they can be suitably engineered in $n$-doped multiquantum-well (MQW) semiconductor heterostructures [5]. Within this platform, by controlling the well and barrier width, the transition energy between electron subbands can be tailored to maximize nonlinear processes of choice. These subbands can also be electrically tuned with an applied voltage bias. The use of these effects has been limited so far by saturation processes, as well as by the difficulty in efficiently coupling light to the proper polarization in order to engage these effects. This is because intersubband transitions in MQWs are excited by an electric field polarized vertically with respect to the MQW layers, which is a challenge in free-space optics, since light is

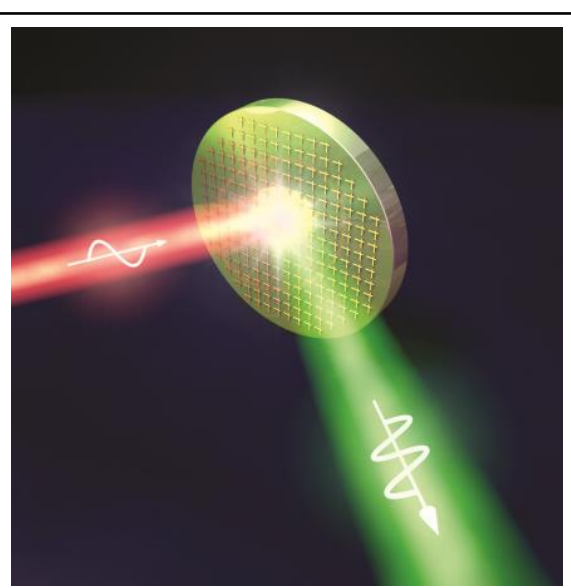

Figure 1 - A nonlinear mirror with large nonlinear conversion efficiency, based on a plasmonic ametasurface loaded by a thin MQW substrate [8]. [Credit for the image to Mr. Erik Zumalt and Prof. Mikhail Belkin, The University of Texas at Austin]

typically transversely polarized and MQWs are grown layer by layer on a substrate.

Yet, these engineered substrates can be of fundamental relevance to overcome the current limitations of nonlinear optics, especially when applied to midinfrared wavelengths, providing unique opportunities to tailor thermal emission, and in various military applications over a frequency range that lacks sources, detectors, and various optical components.

\section{Current and Future Challenges}

As a promising step in this direction, in collaboration with our colleagues at UT Austin and TU Munich we recently proposed a thin 'nonlinear' mirror (see Fig. 1) obtained by loading a $400 \mathrm{~nm}$ thick MQW substrate with a properly designed plasmonic metasurface, resonant in the mid-IR range [8]. The mirror is able to generate second-harmonic reflected waves at $4 \mu \mathrm{m}$ wavelength with very low input intensities, opening the possibility of engaging large nonlinear responses from weak mid-IR sources. Engaging the large MQW nonlinearity, mediated by a suitably designed metasurface, the mirror produces about six orders of magnitude larger conversion efficiencies than natural nonlinear materials of similar thickness. This unprecedented nonlinearity level was achieved by the suitable combination of five parallel mechanisms: (i) proper coupling between the MQW resonance and the electromagnetic resonance sustained by the metasurface; (ii) proper overlap of the resonant modes sustained by the metasurface within the MQW substrate at the fundamental and second-harmonic frequencies; (iii) suitable conversion of the impinging transverse polarization into normal electric field in the substrate, mediated by the plasmonic inclusions forming the surface; (iv) suitable conversion of the 
induced nonlinear polarization currents in the MQW layers into free-space transversely polarized radiation, again mediated by the metasurface inclusions; (v) field enhancement induced in the MQW substrate by the plasmonic inclusions. Phase matching constraints are totally relaxed in this geometry, as the nonlinearity stems from a local response in each deeply subwavelength unit cell. Also Brener's group has shown exciting results in this direction [9-10].

All mentioned mechanisms need to be properly balanced and controlled by material and electromagnetic engineering of the mirror in order to achieve giant nonlinear response. In our first proof-ofprinciple experiment [8], while the measured local nonlinear response was very high for low input intensities, several challenges have been evident. Importantly, the nonlinear response showed signs of fast saturation as the input intensity was increased. Numerical simulations show that the fundamental reason behind this issue lies in a sub-optimal use of the thin material substrate underneath each plasmonic inclusion: most of the nonlinear response can be attributed to the MQW region very close to the plasmonic antennas, while the rest of the thin substrate is not efficiently used. Significantly larger nonlinear responses may be expected with an optimal design, and hotspots near the plasmonic antennas are avoided or become less crucial for nonlinear generation.

Another interesting aspect of MQW substrates is their tunability to an applied dc voltage. Preliminary results in exploiting this feature have been presented in [10] for another MQW-loaded metasurface. The tunability here does not involve carrier injection, as in conventional electro-optic materials, but it is based on a direct modification and spectral tuning of the intersubband transitions, and therefore it can be made in principle very fast. However, this requires a careful design of the modulation network, whose time constant fundamentally limits the overall modulation speed.

\section{Advances in Science and Technology to Meet Challenges}

While saturation effects and losses are expected around the MQW resonance, recent progress in material and metasurface engineering suggests that the conversion efficiencies reported in [8] may be largely improved. Optimal overlap integrals between the fundamental and second-harmonic modes, the use of metamaterial concepts to spread the mode in the MQW layer more uniformly and increase the conversion efficiency, electrical pumping and large doping levels that may be used to produce active intersubband structures with loss-compensation of nonlinear processes, all constitute relevant directions to bring these

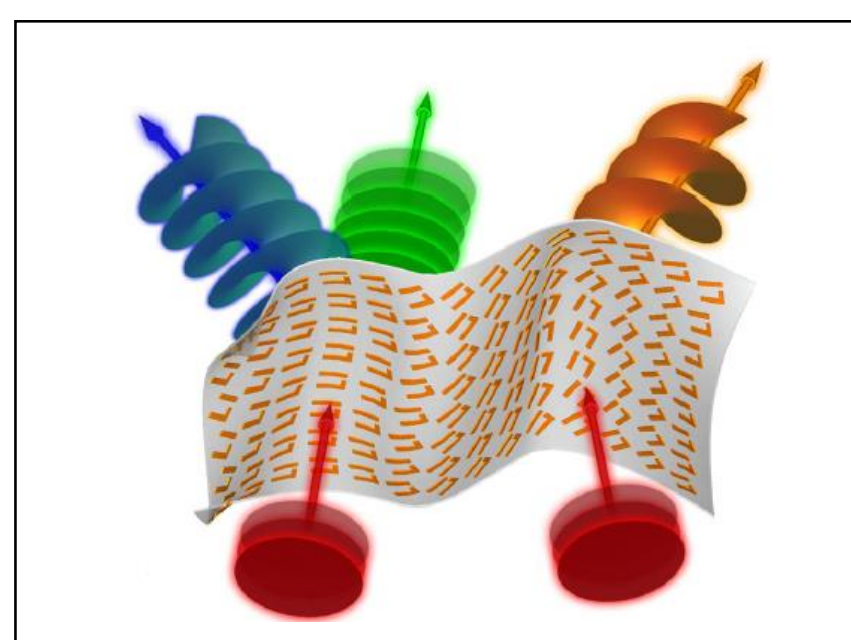

Figure 2 - A metasurface with giant local nonlinear response may offer unique opportunities to pattern, redirect, steer, and modulate the produced nonlinear wavefronts, opening a new paradigm for nonlinear optics. [Credit for the image to Mr. Francesco Monticone, The University of Texas at Austin]

concentrated, giant nonlinear responses to practical use.

Similar concepts may be also translated to visible wavelengths, engaging interband transitions in semiconductors, and the fast modulations supported by these substrates may provide a unique route to realize on-chip isolation using spatio-temporally modulated resonant nanophotonic inclusions [11].

The unprecedented level of concentration of nonlinear response over a deeply subwavelength footprint offered by MQW-loaded metasurfaces may be able to open a new paradigm for nonlinear optics, in which reasonably large conversion efficiencies are expected without the necessity of phase matching [33]-[34]. This implies that it may be possible to pattern the nonlinear wavefront at will over a transmitting surface operated along the same principles. Figure 2 shows this concept, envisioning a tailored pattern of subwavelength inclusions that enhance nonlinear effects of choice, including second-harmonic, difference frequency generation, phase conjugation, and at the same time pattern pixel-by-pixel on a conformal surface the generated wavefront, imprinting polarization, angular momentum, and propagation direction of choice. Being able to tune in real-time the pixel response would provide a reconfigurable nonlinear transmit-array, of interest for various civil and military applications.

\section{Concluding Remarks}

The proposed concept to enhance and engineer the mid-infrared and optical nonlinear response of metasurfaces by the suitable combination of quantum engineered substrates and electromagnetically designed plasmonic inclusions paves the way to several exciting possibilities. At mid-infrared frequencies, it may be 
possible to design compact laser sources and chemical or biomedical sensors. In optics, these concepts may also provide unique opportunities for integrated nanophotonics components with switching and modulation capabilities. This work was supported by the U.S. AFOSR with grant No. FA9550-14-1-0105.

\section{References}

[21] M. Lapine, I. V. Shadrivov, and Y. S. Kivshar, "Colloquium: Nonlinear Metamaterials," Rev. Mod. Phys. 86, 1093-1123 (2014).

[22] M. W. Klein, C. Enkrich, M. Wegener, and S. Linden, "Second-Harmonic Generation from Magnetic Metamaterials," Science 313, 502-504, (2006).

[23] C. Argyropoulos, G. D'Aguanno, and A. Alù, "Giant Second Harmonic Generation Efficiency and Ideal Phase Matching with a Double Epsilon-Near-Zero Cross-Slit Metamaterial," Phys. Rev. B 89, 235401 (2014).

[24] H. Suchowski, K. O’Brien, Z. J. Wong, A. Salandrino, X. Yin, and X. Zhang, "Phase MismatchFree Nonlinear Propagation in Optical Zero-Index Materials," Science 342, 1223 (2013).

[25] E. Rosencher, et al., "Quantum Engineering of Optical Nonlinearities," Science 271, 168-173 (1996).

[26] J. Khurgin, "Second-Order Intersubband NonlinearOptical Susceptibilities of Asymmetric QuantumWell Structures," J. Opt. Soc. Am. B 6, 1673-1682 (1989).

[27] G. Almogy, M. Segev, and A. Yariv, "Adiabatic Nonperturbative Derivation of Electric-Field-Induced Optical Nonlinearities in Quantum Wells," Phys. Rev. B 48, 10950-10954 (1993).

[28] J. Lee, M. Tymchenko, C. Argyropoulos, P. Y. Chen, F. Lu, F. Demmerle, G. Boehm, M. C. Amann, A. Alù, and M. A. Belkin, "Giant Nonlinear Response from Plasmonic Metasurfaces Coupled to Intersubband Transitions," Nature 511, 65-69 (2014).

[29] S. Campione, A. Benz, M. B. Sinclair, F. Capolino, I. Brener, "Second harmonic generation from metamaterials strongly coupled to intersubband transitions in quantum well", Appl. Phys. Lett., vol. 104, p-131104, 2014.

[30] O. Wolf, S. Campione, A. Benz, A. P. Ravikumar, S. Liu, T. S. Luk, E. A. Kadlec, E. A. Shaner, J. F. Klem, M. B. Sinclair, I. Brener, Nat Commun. 2015, 6, 7667.

[31] J. Lee, S. Jung, P. Y. Chen, F. Lu, F. Demmerle, M. C. Amann, A. Alù, and M. A. Belkin, "Ultrafast Electrically-Tunable Polaritonic Metasurfaces," Advanced Optical Materials 2, 1057-1063 (2014).

[32] D. Sounas, and A. Alù, "Angular-Momentum-Biased Nanorings to Realize Magnetic-Free Integrated Optical Isolation," ACS Photonics 1, 198-204 (2014).

[33] M. Tymchenko, J. S. Gomez-Diaz, J. Lee, N. Nookala, M. A. Belkin, A. Alù, "Gradient Nonlinear
Pancharatnam-Berry Metasurfaces," Physical Review Letters 2015, 115, 207403.

[34] J. S. Gomez-Diaz, M. Tymchenko, J. Lee, M. A. Belkin, and A. Alù, "Nonlinear Processes in MultiQuantum-Well Plasmonic Metasurfaces: Electromagnetic Response, Saturation Effects, and Fundamental Limits," Physical Review B, Vol. 92, No. 12, 125429 (13 pages), September 21, 2015. 


\section{Directional control of nonlinear} scattering from metasurfaces - Ekaterina Poutrina and Augustine M. Urbas

Air Force Research Laboratory

\section{Status}

Nonlinear metasurfaces represent a unique class of structures offering the potential for utilizing strong local enhancement of the nonlinear response while minimizing limitations due to linear and nonlinear absorption in the medium. The local efficiency of a nonlinear interaction can be increased by many orders of magnitude owing to strong resonant field enhancements achievable within plasmonic nanocomposites. With a tailored design, the locally enhanced nonlinearly produced field can be re-radiated directly to the far field, with the metasurface acting as an array of nonlinear optical antennas. Indeed, enhanced second harmonic generation has been demonstrated in a metasurface formed by an array of split ring resonators [1]; up to six orders of magnitude increase in power flow of the nonlinearly generated field have been predicted (Fig.1) [2] and demonstrated experimentally [3] for the third order nonlinear response in the geometries of gold nanowires or nanostrips coupled to a gold film, compared to that achievable from a plain gold film; a dipolar resonatorbased metasurface had been predicted [4] to enhance nonlinear imaging aiming at a sub-diffraction resolution [5]; while local field enhancements at the fundamental and the second harmonic frequencies have been used to improve the second-order nonlinear response from intersubband transitions in semiconductors, at the same time demonstrating the capability of engineering an arbitrary component of the effective nonlinear susceptibility tensor [6]. In addition to the enhanced efficiency of nonlinear generation, a variety of other unique features are offered by the nonlinear response of these two-dimensional structures. Owing to their deeply-subwavelength thickness, phase matching constraints are removed, allowing the simultaneous and nearly-equally efficient conversion of a wide range of $k$-vectors of the fundamental field to a set of k-vectors of the nonlinearly generated light, including a direct coupling between evanescent and propagating waves $[2,7]$. The latter creates opportunity for a subdiffraction imaging [5, 8], as well as for an efficient coupling between the in-plane and out-of-plane modes $[2,7]$. Furthermore, the inherent intensity-dependence of the nonlinear response allows for a dynamic, realtime control of any of the above operations, as well as for a directionality control achieved with planar geometries. Indeed, a dynamic, noncontact tuning of focusing and defocusing has been demonstrated using a specially-designed illumination intensity profiles [9].

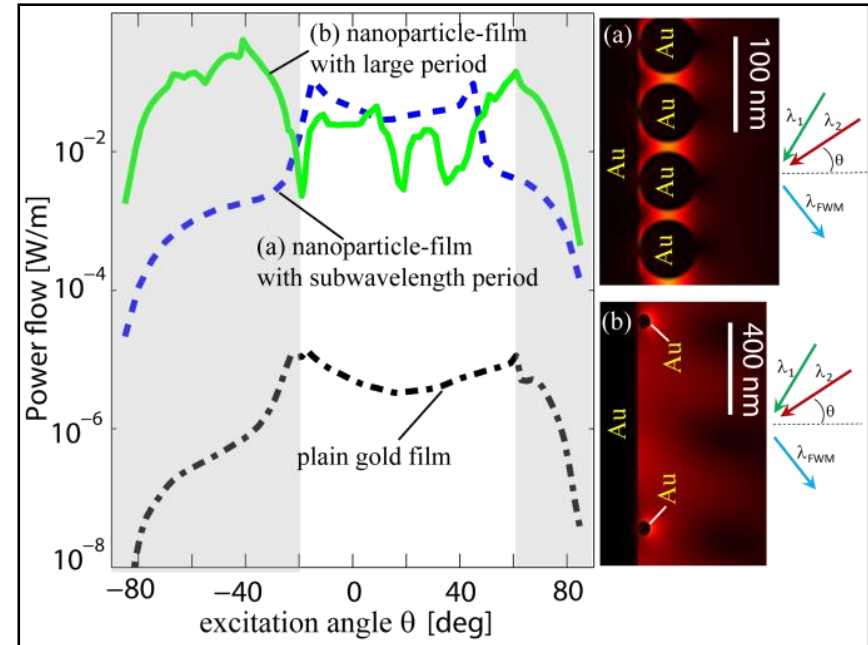

Fig. 1. Numerical demonstration of an up to 6 orders of magnitude increase in power flow of the four-wave mixing (FWM) process on scattering from a nanowirefilm geometry (insets on the right), compared to a plain gold film (after Ref. [3]). The shaded regions show the evanescent regime of FWM generation in the case of a plain film. A large-period geometry allows outcoupling of the enhanced evanescent field into the far field producing a stronger power flow in these regions.

Such strong enhancements of the nonlinear response achievable with subwavelength-thin structures, combined with the option of optically-controlled propagation and in-plane/out-of-plane coupling, are unique and open possibilities for a tremendous variety of applications in integrated photonics and quantum information processing. The $2 \mathrm{D}$ nature of the geometry, on the other hand, considerably simplifies the fabrication procedure compared to its bulk counterpart.

\section{Current and Future Challenges}

However, control of light interaction on the nanoscale faces numerous limitations unique to the microscopic nature of the processes involved. One of the major limiting factors is the reduction of the degree of coherence occurring with the interaction of even a highly coherent light beam with a nanoscale object. The latter results in a significant reduction of directionality control: while focusing and re-routing of light are routine procedures with macroscopic optics, diffraction dominates on the nanoscale level. The problem persists in the case of a nonlinear interaction in nanostructures,, where the interaction volume is significantly below of what would allow for a noticeable phase-matching impact. The absence of phase matching requirement, while relaxing the conditions for the manifestation of any nonlinear process, eliminates the major tool governing both the direction of the nonlinearly produced light and the choice of the dominating process. As a result, for a 
single nanoelement, the nonlinear generation occurs nearly isotopically in all directions, for each process involved. While arranging the elements in a metasurface allows interferometric cancelation of nonlinear generation in all but one direction defined, in this case, by the boundary conditions at the interface, the generation is still symmetric on each side of the surface, producing nearly equal amounts of light in each hemisphere. Achieving directionality control is thus one of the main challenges for efficient utilization of light produced with such an enhanced nonlinear interaction.

\section{Advances in Science and Technology to Meet Challenges}

A logical path to regaining the control of directionality relies on exploiting the processes inherent to nanoscale interactions, such as interference effects between multipole modes of the electromagnetic field produced within a scattering event. Orthogonal, spatially superimposed multipoles of opposite parities can interfere destructively in one direction while doubling the scattered field amplitude in the opposite direction, thus producing a directional response on the nanoscale [10]. While linear directional scattering from metasurfaces has been studied intensively [11], many topics in the case of a nonlinear response are yet to be addressed. The first analyses, however, have indicated unparalleled features brought by nonlinear magnetoelectric interference in handling the directionality of nonlinear generation [11-13]. Nonlinear light generation by a nanoelement can provide the unique opportunity of a direct observation of the electromagnetic modes possessed by that element: while the total field distribution results from the interference between the scattered and the incident fields in the case of a linear response, a pure (nonlinearly) scattered field is observed via nonlinear generation at a distinct frequency. Moreover, for nanoscale geometries, the orientation of the multipolar components generated with the nonlinear interaction can be decoupled from the excitation direction of the fundamental beam. While, by itself, such a decoupled response contributes little to the unidirectionality of the resulting angular distribution of the nonlinearly generated light, a qualitative difference is brought by magneto-electric interference between the nonlinearly produced multipole partial waves. The latter not only, similar to the linear case, can result in nonlinearly produced field acquiring a certain dominant direction, but also allows for this direction to be decoupled from that of at least one of the excitation beams, making the directionality of the response effectively non-reciprocal with respect to that beam $[13,14]$. When arranged in a metasurface with a proper nanoelements orientation within the surface plane and tailored spectral positions

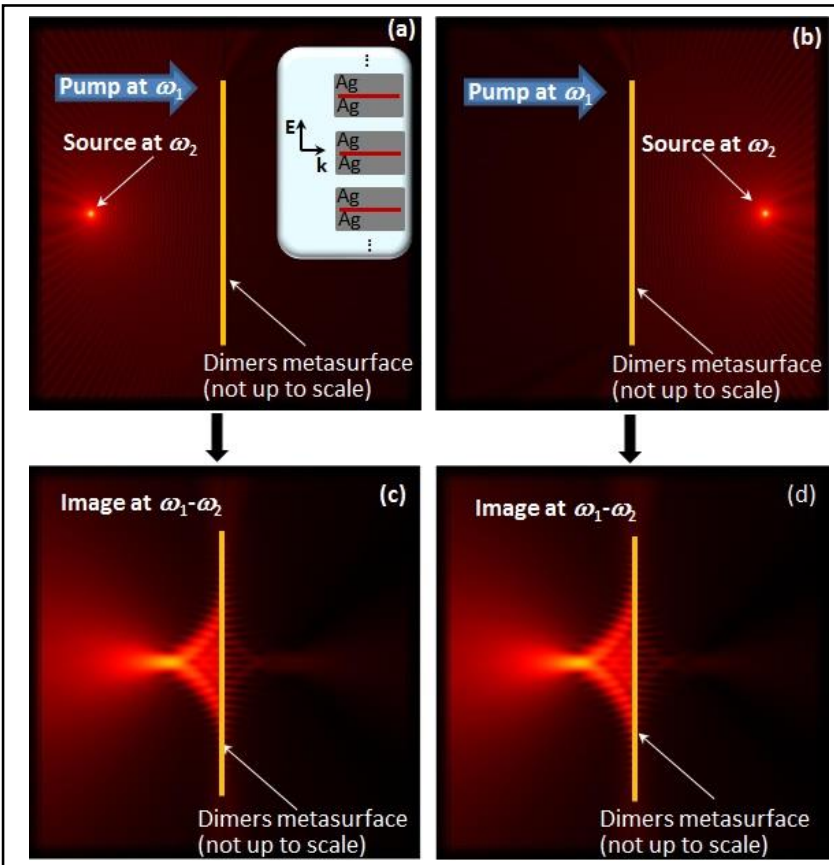

Fig. 2 - An example of a "non-reciprocal" nonlinear imaging, with image generation on the same (left) side of the metasurface independently of the source location [13]. Inset in (a) shows assumed metasurface geometry [9].

of the excitation beams with respect to nanoelement resonances, the produced directional beam can maintain its "non-reciprocal" nature. The latter brings an option of "non-reciprocity" to virtually any application that utilizes the specified nonlinear interaction in such a metasurface. A numerical example of a "non-reciprocal" nonlinear imaging produced by the process of difference frequency generation in a metasurface made of periodically arranged plasmonic dimers, having the geometry parameters similar to the those given in [11], is shown in Fig. 2. The described response, however, depends on numerous factors, including the crystalline properties of the constituting materials, as well as the linear multipolar response of the particular nanoelement geometry employed. Further analysis of the impact of the above factors, development of a procedure for the effective hyperpolarizabilities retrieval, similar as it was done in the linear case [15], and a subsequent optimization of the geometry for fabrication, have therefore yet to be performed.

\section{Concluding Remarks}

The results outlined above clearly demonstrate a unique potential of metasurfaces for providing a strongly enhanced and directional nonlinear response. Moreover, non-reciprocity in the direction of nonlinear generation -- a phenomena fundamentally impossible in the linear response and unachievable in the nonlinear response of conventional optical materials--is inherent and predicted to be realistically observable in 
the effective nonlinear multipolar response of nanostructures. Future experimental implementations of this phenomenon, combined with the strong enhancement of the nonlinear response offered by metasurfaces, the option of a dynamic control of the directionality, as well as the overall deeply miniature dimensions of these structures offer key features for an immense variety of applications in integrated optics and photonics.

\section{References}

[1] M.W. Klein, C. Enkrich, M. Wegener, and S. Linden, "Second-harmonic generation from magnetic metamateials", Science 313, 502-504 (2006).

[2] E. Poutrina, C. Ciracì, D. J. Gauthier, and D. R. Smith, "Enhancing four-wave mixing processes by nanowire arrays couled to a gold film", Opt. Express 20, 11005-11013 (2012).

[3] J. B. Lassiter, X. Chen, X. Liu, C. Ciracì, T. B. Hoang, S. Larouche, S.-H. Oh, M. H. Mikkelsen, and D. R. Smith, "Third harmonic generation enhancement by film-coupled plasmonic stripe resonators", ACS Photonics 1, 1212-1217 (2014).

[4] P.-Y. Chen and Andrea Alù, "Subwavelength imaging using phase-conjugating nonlinear nanoantenna arrays" 11, 5514-5518 (2011).

[5] J. B. Pendry, "Time Reversal and Negative Refraction", Science 322, 71-73 (2008).

[6] J. Lee, M.Tymchenko, C. Argyropoulos, P.-Y. Chen, F. Lu, F. Demmerle, G. Boehm, M.-C. Amann, A.Alu' and M. A. Belkin, "Giant nonlinear response from plasmonic metasurfaces coupled to intersubband transitions", Nature 511, 65-69 (2014).

[7] J. Renger, R. Quidant, N .van Hulst, and L. Novotny, "Surface-Enhanced Nonlinear Four-Wave Mixing”, Phys. Rev. Lett. 104, 046803 (2010).

[8] S. Palomba, S. Zhang, Y. Park, G. Bartal, X. Yin, and $\mathrm{X}$. Zhang, "Optical negative refraction by fourwave mixing in thin metallic nanostructures", Nat. Mater. 11, 34 (2012).

[9] I. V. Shadrivov, P. Kapitanova, S. I. Maslovski, and Y. S. Kivshar, "Metamaterials controlled with light”, Phys. Rev. Lett. 109, 083902 (2012).

[10] M. Kerker, D.-S. Wang, and C. L. Giles, "Electromagnetic scattering by magnetic spheres," J. Opt. Soc. Am. 73,v765 (1983).

[11] E. Poutrina, A. Rose, D. Brown, A. M. Urbas, and D. R. Smith "Forward and backward unidirectional scattering from plasmonic coupled wires" Opt. Express 21, 31138-31154 (2013), and references therein.

[12] A. Rose, S. Larouche, E. Poutrina, and D. R. Smith, "Nonlinear magnetoelectric metamaterials: analysis and homogenization via a microscorpic coupled-mode theory", Phys. Rev. B 86, 033816 (2012).
[13] E. Poutrina and A. M. Urbas, "Non-reciprocal nonlinear imaging with dimer-based metasurfaces", The 9th International Congress on Advanced Electromagnetic Materials in Microwaves and Optics, Metamateials' 2015, Oxford, London.

[14] E. Poutrina and A. M. Urbas, "Multipole interference for non-reciprocal nonlinear generation", arXiv:1510.03012 (in review).

[15] E. Poutrina and A. Urbas, "Multipole analysis of unidirectional light scattering from plasmonic dimers" J. Opt. 16, 114005 (15 pages) (2014). 


\section{Nonlinear properties of optical metamaterials - Natalia M. Litchinitser}

University at Buffalo, The State University of New York

\section{Status}

Nonlinear optics is a fascinating branch of science that focuses on light propagation in media, in which the dielectric polarization responds nonlinearly to the electric and/or magnetic field of light. Although this field has been developing for decades, nonlinear optical materials available to date are still limited by either slow material response time in such phenomena as saturable absorption, photorefractive effect, and thermal nonlinear phenomena, or by relatively low and generally band-limited nonlinear susceptibilities responsible for ultrafast nonlinear processes. While thermally nonlinearities such as thermally induced metal-to-insulator transition in vanadium dioxide and thermo-optical effects in silicon result in refractive index changes of the order of 1 , the response time is slow. On the other hand, it is difficult to find any ultrafast nonlinear material that is capable of achieving an ultrafast refractive index change larger than a fraction of a percent. For decades, scientists have been exploring ways of creating materials with a large, fast, and broadband nonlinear response; if found, these would revolutionize nonlinear optics, leading to lowpower, compact, and ultra-fast applications of nonlinear optical phenomena.

\section{Current and Future Challenges}

Over the fifty years of the development of the field of nonlinear optics, significant theoretical progress has been made in understanding the origin and limitations of nonlinear responses. In parallel, a number of practical applications have emerged, such as optical parametric generation, mode locking, and comb generation. Yet one of the most essential functionalities -all-optical switching- has not been fully realized because the nonlinear properties of materials that have been explored to date are too small to support efficient all-optical switching. After many years of active research, the best results have been demonstrated in optical fibers, owing to long propagation length rather than their nonlinear properties.

The emergence of metamaterials has a potential to advance the development of such materials. Indeed, metamaterials were predicted to enable a number of novel linear and nonlinear light-matter interactions, including magnetic nonlinear response, backward phase-matching, and the nonlinear mirror, recently demonstrated at microwave frequencies [1].

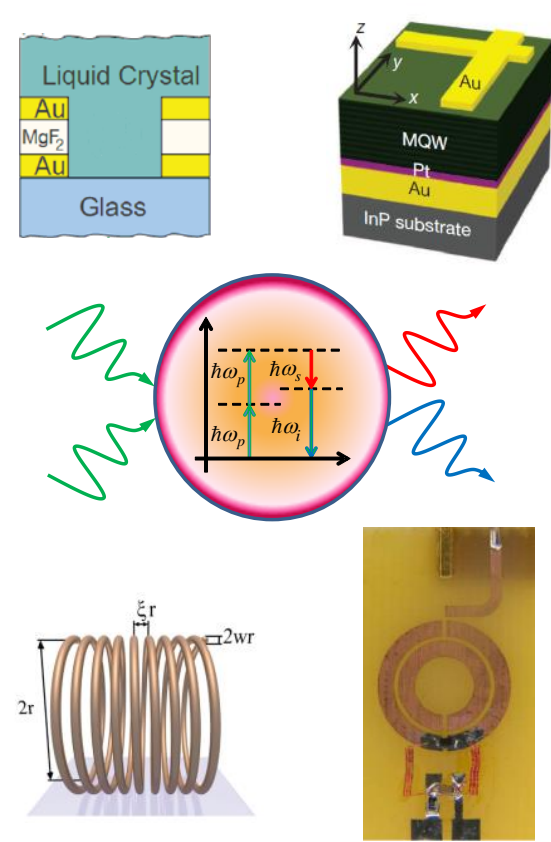

Figure 1. Nonlinear meta-atoms proposed and demonstrated for metamaterials-enhanced nonlinear optics.

Since it has already been demonstrated that linear optical properties, such as dielectric permittivity, magnetic permeability, and refractive index of metamaterials can be designed to be positive, negative or even zero at any selected frequency by properly adjusting the dimensions, periodicity and other properties of the so-called meta-atoms (the unit cells of metamaterials), it is expected to be possible to largely transform nonlinear properties of metamaterials. Figure 1 shows several examples of nonlinear meta-atoms proposed to date.

In one of the first theoretical studies, the nonlinear response of metamaterials was analyzed for the particular case of a two-dimensional periodic structure created by arrays of wires and split-ring resonators embedded into a nonlinear dielectric host material [2]. The nonlinear response in this structure was shown to have two different contributions. The first contribution originates from an intensity-dependent part of the effective dielectric permittivity of the nonlinear dielectric host material, and the second contribution comes from the periodic structures of resonators that lead to intensity-dependent magnetic response. The magnetic nonlinearity has been found to be much stronger than the nonlinearity in the dielectric properties due to the field enhancement in the split-ring resonators. Also, demonstrated in the microwave frequency range was the possibility of tuning the properties of split-ring resonator-based metamaterials at the level of the individual meta-atom. 
At optical frequencies, in the related field of plasmonics, significant efforts have been devoted to the enhancement of nonlinear optical response using various plasmonic nanostructures, including metallic bow-tie nano-antennas, dimers, trimers, and other structures sometimes referred to as plasmonic metamaterials. While these first steps demonstrate the feasibility of locally enhancing the nonlinear response, it is important to note that a majority of these studies essentially exploited strong local field enhancements enabled by plasmonic (metallic) nanostructures rather than actual rational design of the nonlinear optical response of the metamaterial. There are several limitations associated with such an approach [3]. First, the majority of approaches rely on strong local field enhancement in metal-dielectric (plasmonic) nanostructures leading to very large local effective nonlinear refractive index, while strong absorption in the metal limits the attainable nonlinear phase shift (in the case of self- and cross-phase modulation effects) and frequency conversion efficiency (in the case of four-wave mixing) at very short distances. It should be noted that local field enhancements are not unique to metamaterials and have been previously realized in many other structures, including photonic bandgap structures, micro-cavities, and ring resonators. Moreover, despite many impressive demonstrations of various high-Q resonantly enhanced structures, careful analysis suggests that they are not as promising as was initially hoped for nonlinear optical processes requiring high efficiency such as wavelength conversion or switching.

\section{Advances in Science and Technology to Meet Challenges}

The development of nonlinear metamaterials is likely to impact both the nonlinear optics and metamaterials fields by enabling entirely new phenomena. In particular, the novel optical properties facilitated by the metamaterials field prompt us to reconsider many nonlinear light-matter interactions, including SHG, parametrical amplification, ultra-short pulse dynamics, and soliton propagation [4-7]. From an applications viewpoint, nonlinear metamaterials make possible creating dynamically-tunable materials, optical switches, filters, beam deflectors, focusing/defocusing reflectors, and reconfigurable structures with controlled transparency, refractive index, and nonlinear response. However, in order to transition academic research developments to industrial scale production, several issues will need to be addressed. These include the development of large scale, inexpensive fabrication approaches and material systems compatible with existing platforms, such as complementary metal-oxide semiconductor technology.

Recently, a number of nonlinearly-enabled functionalities and devices were proposed [8-10], including nonlinear cloaking devices, reconfigurable nonlinear light concentrators, and the light-tunable reflection, shaping, and focusing of EM waves in metamaterials. As an example let's consider an electromagnetic wave concentrator with a core comprised of focusing Kerr nonlinearity. Figure 2 shows two possible configurations of the nonlinear concentrator. The basic principle of the device is based on a combination of transformation optics design of the outer shell of the cylindrical concentrator made of linear graded-index MM, and nonlinear changes in the refractive index of the inner region that results from nonlinear correction to the linear

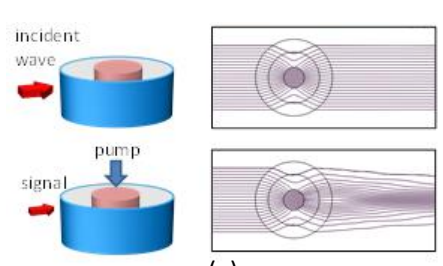

(a)

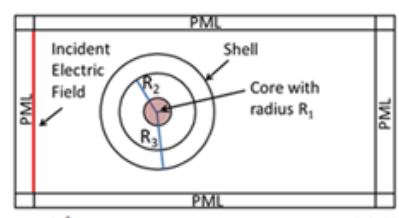

(b)

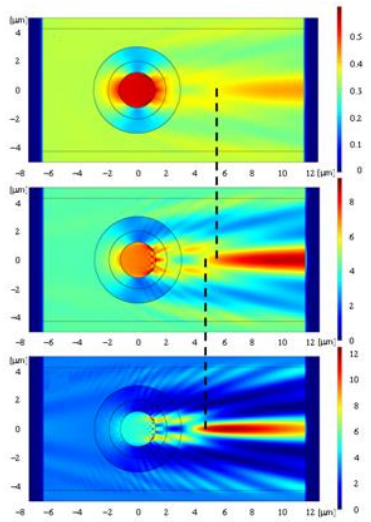

(c)
Figure 2. (a) Schematic of a wave concentrator based on selfaction of single high intensity beam (top left), nonlinear concentrator for a weak signal propagating $\mathrm{n}$ nonlinear medium with refractive index modified by a strong pump (bottom left), and power flow in a linear (top right) and nonlinear concentrator based on self-action (bottom right). (b) Schematic of a lens. (c) Time averaged power flow illustrating the predicted lensing effect in the case of focusing nonlinearity. Focal line shifts towards the lens as incident field increases.

refractive index due to Kerr nonlinearity. A simple physical picture of performance of the device shown in Fig. 2(a) (top left)-an axicon-like lensing with a variable focus line, can be gained from a simple ray picture of light refraction at the shell-core interface or by considering a power flow inside and outside of the structure, as shown in Fig. 2(a) (right). In the case of defocusing Kerr nonlinearity, the refractive index of the core would decrease with increasing intensity of an incoming beam, leading to the beam defocusing/splitting effect.

\section{Concluding Remarks}

Current nonlinear optical materials are generally limited by either slow material response time, thermal nonlinear phenomena, or by relatively low and band-limited nonlinear susceptibilities. The development of novel nonlinear metamaterials is expected to enable entirely new phenomena leading to new functionalities and devices.

\section{References}

[1] A. Rose et al., Phys. Rev. Lett. 107, 063902(4) (2011).

[2] A. A. Zharov et al., Phys. Rev. Lett. 91, 037401(4)

(2003).

[3] J. B. Khurgin and G. Sun, Opt. Express 21, 27460-27480 (2013).

[4] J. B. Pendry, et al., IEEE Trans. Microw. Theory Tech. 47, 1999, 2075-2084 (1999).

[5] M. Scalora, et al., Phys. Rev. Lett. 95, 013902(4) (2005).

[6] A. K. Popov, and V. M. Shalaev, Appl. Phys. B, 84,

131-137 (2006).

[7] E. Poutrina et al., New J. Phys. 12, 093010(27) (2010).

[8] N. A. Zharova, et al., Opt. Express 20, 2012, 14954-

14959 (2012).

[9] A. Pandey, and N. M. Litchinitser, Nonlinear light concentrators, Opt. Lett. 37, 5238-5240 (2012). 
[10] I. V. Shadrivov et al., Phys. Rev. Lett. 109, 083902(4) (2012). 


\section{Optical gain in plasmonics and metamaterials - Mikhail A. Noginov Norfolk State University}

\section{Status}

As it has been argued in the previous sections of this paper, photonic metamaterials, whose properties often critically depend on localized plasmon oscillations and propagating surface plasmon waves, can manipulate light in unprecedented ways, enabling scores of novel phenomena and applications ranging from negative index of refraction and invisibility cloaking to control of spontaneous emission and the nanocircuitry operating at optical frequencies. However, most metamaterial and plasmonic structures have one common problem that dramatically limits their performance - absorption loss in metal (or any other plasmonic material with negative dielectric constant). At the same time, until recently, most metamaterial and plasmonic systems were passive. On the other hand, future meta-devices and meta-systems require not only passive elements (analogous to resistors, capacitors and inductances in conventional electronic circuits) but also active control elements that would play a role of modern transistors or historic vacuum tubes.

Optical gain introduced to metamaterials and plasmonic systems can be a solution to both challenges outlined above. As it has been proposed in Ref. [1], by adding optical gain to a dielectric adjacent to the metallic surface, one can compensate propagation loss of surface plasmon polaritons (SPPs) and, if gain exceeds the loss, obtain a stimulated emission of SPPs. Nearly fifteen years later, stimulated emission of localized surface plasmons (SPs) - known as the spaser effect - has been predicted in Ref. [2].

Experimentally, partial (very small) compensation of the SPP propagation loss at the visible wavelength by gain in an optically pumped laser dye has been first demonstrated in Ref. [3]. A much stronger compensation of the SPP loss by gain has been further shown by multiple groups in a variety of systems, see e.g. Ref. [4] (Fig 1a). This research has culminated in the demonstration of stimulated emission of SPPs $[5,6]$ with several different feedback mechanisms (Fig. 1b) and a spaser effect in gold nanoparticles coated by dyeimpregnated silica [7], Fig. 1c.

Several examples above highlighted loss compensation and stimulated emission in the simplest plasmonic systems. Systematic theoretical and experimental studies of the compensation of loss by gain in complex metamaterial structures have shown that a relatively small gain can provide substantial loss compensation if it is strategically placed in the electric field hot spots [8]. Consequently, full loss compensation has been

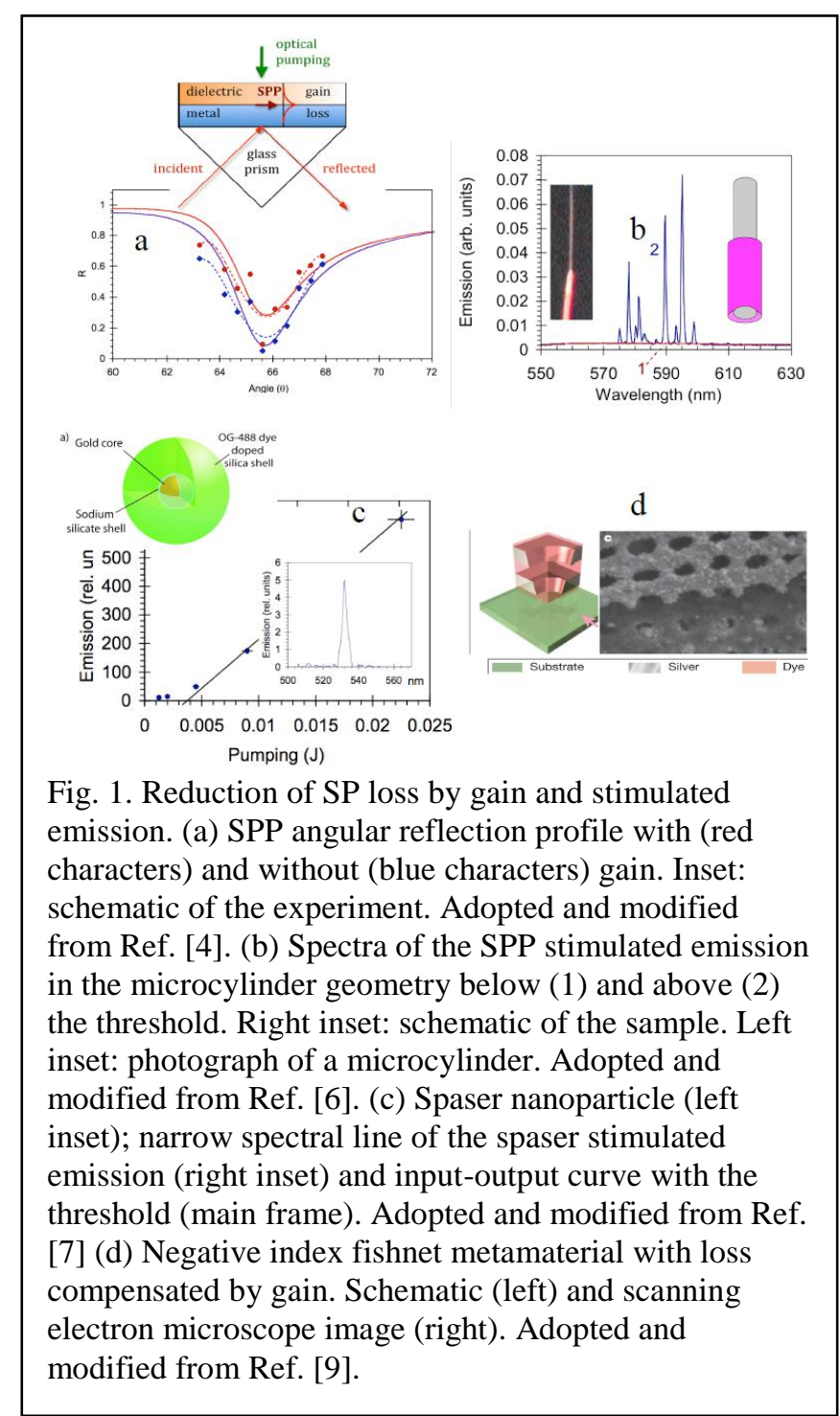

demonstrated in the visible spectral range in the negative index fishnet metamaterial [9], Fig. 1d.

\section{Current and Future Challenges}

Loss compensation and stimulated emission in plasmonic structures and metamaterials face a number of challenges at both fundamental and technological levels.

(1) The gain required to compensate SP loss and achieve stimulated emission is too high, $\geq 1000 \mathrm{~cm}^{-1}$ for SPPs and $\geq 10,000 \mathrm{~cm}^{-1}$ for localized SPs.

(2) Optical pumping with nanosecond Q-switched laser pulses is impractical and electrical pumping would require the very high current, $\leq 50 \mu \mathrm{A}$ [10], which will damage the structure. (However, the threshold current can be reduced dramatically, to $\sim 3 \mu \mathrm{A}$, as the size of the cavity approaches $\lambda / 2$ [11].)

(3) Dye molecules can provide optical gain that, arguably, extends to the $\geq 100,000 \mathrm{~cm}^{-1}$ range (in the absence of quenching). However, they have low 
damage threshold and cannot sustain strong laser pumping for a long time. Quantum dots (QDs) have been reported to have emission cross sections as high as $7 \times 10^{-15} \mathrm{~cm}^{2}$ [12] (at the level of the best performing dye molecules). However, the reported experimental values of the optical gain in the QD based amplifying media do not exceed $1000 \mathrm{~cm}^{-1}$ [13]. However, a quantum well/quantum cascade plasmonic laser has been successfully demonstrated in the infrared [14]. However, SP loss compensation by quantum wells in the visible range remains problematic, despite promising theoretical predictions and demonstrated gain of $\sim 2,000 \mathrm{~cm}^{-1}$ in one system[15].

(4) Although the theoretical model exists for a spaser generating coherent localized surface plasmons $[2,16,17]$, the authors are not aware of a comprehensive theory describing a laser based on propagating SPs (including dynamics of population inversion, energy transfer to metal and effect of the density of photonic states).

(5) High gain requires high concentration of dye molecules or quantum dots with high emission (and absorption) cross sections. Strong coupling of such molecular ensembles with surface plasmons is known to strongly distort and split the SP dispersion curve $[18,19]$. However, this effect has been neglected at the analysis of the surface plasmon stimulated emission. (Cooperative emission of dye molecules enabled by localized SP resonance in matellic nanoparticles has been predicted in Ref. [20].)

\section{Advances in Science and Technology to Meet Challenges}

As follows from the discussion above, many challenges encountered by active plasmonics and metamaterials would diminish or disappear at reduced loss in plasmonic media. Thus, it has been recently demonstrated that the lasing threshold of SPPs can be reduced by more than an order of magnitude if the stimulated emission wavelength is shifted from 0.60 $\mu \mathrm{m}$ to $0.87 \mu \mathrm{m}$ [21], Figs. 2a and 2b. Even further reduction of the stimulated emission threshold can be achieved if surface plasmons propagate on top of lamellar metal-dielectric metamaterials with hyperbolic dispersion and high density of photonic states [22].

Significant efforts have been aimed at reduction of loss in plasmonic media. Of particular interest are the studies focused at replacing metals with transparent conductive oxides, e.g. Indium Tin Oxide (ITO) and Aluminum doped Zinc Oxide (AZO), as well as other non-metallic materials (such as TiN), providing plasmonic responses in the visible and near infrared ranges of the spectrum [23]. A key breakthrough in SP loss management has occurred within the past several years, when high-quality epitaxial silver films were

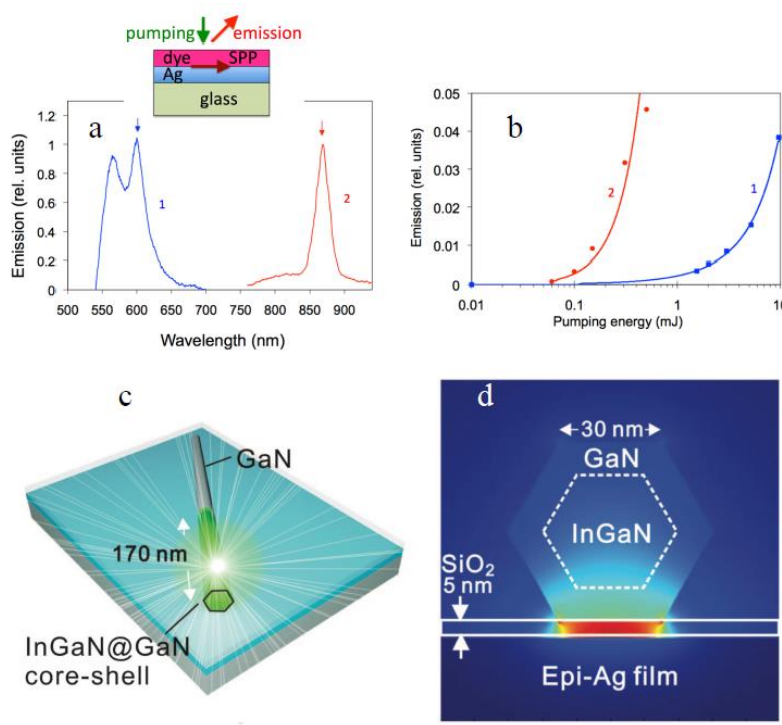

Fig. 2. Reduced threshold of the SPP stimulated emission. (a) Spectra of the visible (1) and near infrared (2) SPP stimulated emission enabled by the R6G and HITC laser dyes, respectively. (Inset: schematic of the experiment.) (b) Corresponding input/output curves showing $\sim 20$-fold reduction of the stimulated emission threshold in the near infrared. Arrows indicate stimulated emission wavelengths. Adopted and adapted from Ref. [21]. (c) Schematic of the SP nanolaser consisting of a single InGaN@GaN core-shell nanorod on a SiO2covered epitaxial Ag film. (d) Calculated energy-density distribution of the stimulated emission mode. Adopted from Ref. [24].

demonstrated [24,25] and shown to enable lowthreshold SP stimulated emission [24], Figs 2c and 2d. Very recently, sputtered Ag films were reported to support SPPs propagating $\geq 100 \mu \mathrm{m}$ at $\lambda \geq 650$ [26].

To the disappointment of many researchers, the SP absorption loss in the "conventional quality" [27] silver films shows only minor reduction at cryogenic temperatures, since scattering on defects and grain boundaries is larger than scattering on phonons. A more significant reduction of the SP loss at low temperatures can be expected in high quality silver films discussed above.

Metamaterials are often highly anisotropic and have strongly different propagation constants (for light or plasmons) in different directions. Thus, in metamaterials with hyperbolic dispersion, such frequency-dependent low-loss directions, which are favorable for compensation of loss by gain, stimulated emission, and active control of metamaterials and devices, have been identified in Refs. [29-31].

\section{Concluding Remarks}

Optical gain inside or in the vicinity of metamaterials and plasmonic structures can compensate loss as well as provide for stimulated emission and active control. Gain provided by optical pumping enabled numerous 
proof-of-principle demonstrations. Unfortunately, it is impractical for the majority of applications. Electrically pumped gain can revolutionize plasmonic, metamaterial and nanophotonics technologies. However, it requires lower loss than is available in conventional plasmonic materials.

The recently demonstrated: (i) strong reduction of loss in epitaxial and sputtered Ag films, (ii) shift to the near infrared spectral range (when applications allow), and (iii) use of lamellar metal-dielectric structures instead of single thick metallic films can potentially reduce the loss to a level at which it can be further controlled by electrically pumped gain. The benefits of electrically controlled plasmonic and metamaterial systems to a range of disruptive technologies (briefly outlined in the previous sections of this article) justify the grand-scale effort.

\section{References}

[1] Sudarkin A N and Demkovich P A 1989 Excitation of surface electromagnetic waves on the boundary of a metal with an amplifying medium Sov. Phys. Tech. Phys. 34 764-766

[2] Bergman D J and Stockman M I 2003 Surface plasmon amplification by stimulated emission of radiation: quantum generation of coherent surface plasmons in nanosystems Phys. Rev. Lett. 90027402

[3] Seidel J, Grafstroem S and Eng L 2005 Stimulated emission of surface plasmons at the interface between a silver film and an optically pumped dye solution. Phys. Rev. Lett. 94177401.

[4] Noginov M A, Podolskiy V A, Zhu G, Mayy M, Bahoura M, Adegoke J A, Ritzo B A and Reynolds K 2008 Compensation of loss in propagating surface plasmon polariton by gain in adjacent dielectric medium Opt. Express 16 1385-92

[5] Noginov M A, Zhu G, Mayy M, Ritzo B A, Noginova N and Podolskiy V A 2008 Stimulated emission of surface plasmon polaritons Phys. Rev. Lett. 101226806

[6] Kitur J K, Podolskiy V A and Noginov M A 2008 Stimulated emission of surface plasmon polaritons in microcylinder cavity Phys. Rev. Lett. 106183903

[7] Noginov M A, Zhu G, Belgrave A M, Bakker R, Shalaev V M, Narimanov E E, Stout S, Herz E, Suteewong T and Wiesner U 2009 Demonstration of a spaser-based nanolaser Nature 460 1110-12

[8] Sivan Y, Xiao S, Chettiar U K, Kildishev AV and Shalaev V M 2009 Frequency-domain simulations of a negative-index material with embedded gain. Opt. Express 17 24060-74

[9] Xiao S, Drachev V P, Kildishev A V, Ni X, Chettiar U K, Yuan H K and Shalaev V M 2010 Lossfree and active optical negative-index metamaterials Nature 466 735-38
[10] Khurgin J B and Sun G 2012 Injection pumped single mode surface plasmon generators: threshold, linewidth, and coherence Opt. Express 20 15309-25

[11] M. T. Hill, Oei Y-S, Smalbrugge B, Zhu Y, de Vries $\mathrm{T}$, van Veldhoven $\mathrm{P} \mathrm{J}$, van Otten $\mathrm{F}$ W $\mathrm{M}$, Eijkemans T J, Turkiewicz J P, de Waardt H, Geluk E J, Kwon S-H, Lee Y-H, Nötzel R and Smit M K 2007 Lasing in metallic-coated nanocavities Nature Photonics 1 589-94

[12] Eliseev P G, Li H, Liu G T, Stintz A, Newell T C, Lester L E and Malloy K J 2000 Optical gain in InAs/InGaAs quantum-dot structures: Experiments and theoretical model Quantum Electronics 38 664-668 [13] Klimov V I, Mikhailovsky A A, Xu S, Malko A, Hollingsworth J A, Leatherdale C A, Eisler H-J and Bawendi M G 2000 Optical Gain and Stimulated Emission in Nanocrystal Quantum Dots Science 290 314-17

[14] Sirtori C, Gmachl C, Capasso F, Faist J, Sivco D L, Hutchinson A L and Cho A Y 1998 Longwavelength $(\lambda \square \approx 8-11.5 \mu \mathrm{m})$ semiconductor lasers with waveguides based on surface plasmons. Opt. Lett. 23 1366-68

[15] Alam M Z, Meier J, Aitchison J S, and Mojahedi M 2007 Gain assisted surface plasmon polariton in quantum wells structures Opt. Express 15 176-82 [16] Stockman M I 2010 The spaser as a nanoscale quantum generator and ultrafast amplifierJournal of Optics 12024004

[17] Zheludev N I, Prosvirnin S L, Papasimakis No and and Fedotov V A 2008 Lasing spaser Nature Photonics 2 351-54

[18] Bellessa J, Bonnand, Plenet C J C and Mugnier J 2004 Strong Coupling between Surface Plasmons and Excitons in an Organic Semiconductor Phys. Rev. Lett. 93036404

[19] Fofang N T, Park T-H, Neumann O, Mirin N A, Nordlander P and Halas N J 2008 Plexcitonic Nanoparticles: Plasmon-Exciton Coupling in Nanoshell-J-Aggregate Complexes Nano Lett. 8 348187

[20] Pustovit V N and Shahbazyan T V 2009 Cooperative emission of light by an ensemble of dipoles near a metal nanoparticle: The plasmonic Dicke effect Phys. Rev. Lett. 102077401

[21] Kitur J K, Zhu G and Noginov M A 2014 Lowthreshold stimulated emission of surface plasmons polaritons J. Opt. 16114020

[22] Kitur J K, Tumkur T U and Noginov M A 2014 Stimulated emission of SPPs on top of hyperbolic metamaterials Conference on Lasers and ElectroOptics (CLEO) FTu3C.1

[23] Boltasseva A and Atwater H A 2011 Low-Loss Plasmonic Metamaterials Science 331 290-91 
[24] Lu Y-J, Kim J, Chen H-Y, Wu C, Dabidian N, Sanders C E, Wang C-Y, Lu M-Y, Li B-H, Qiu X, Wen-Hao Chang W-H, Chen L-J, Shvets G, Shih C-K and, Gwo S 2012 Plasmonic Nanolaser Using Epitaxially Grown Silver Film Science 337 450-53 [25] Wu Y, Zhang C, Estakhri N M, Zhao Y, Kim J, Zhang M, Liu X-X, G. K. Pribil, Alù A, Shih C$\mathrm{K}$ and Li X 2014 Intrinsic Optical Properties and Enhanced Plasmonic Response of Epitaxial Silver Advanced Materials 26 6106-10

[26] High A A, Devlin R C, Dibos A, Polking M, Wild D S, Perczel J, de Leon N P, Lukin M D, and Park H 2015, Visible-frequency hyperbolic metasurface, Nature 522, 192-96

[27] Johnson P B and Christy R W 1972 Optical constants of the noble metals Phys. Rev. B 4370

[28] Mayy M, Zhu G, Mayy E, Webb A, Noginov M A 202 Low temperature studies of surface plasmon polaritons in silver films, J. Appl. Phys. 111094103 [29] Argyropoulos C, Nasim Mohammadi Estakhri N M, Monticone F and Alù A 2013 Negative refraction, gain and nonlinear effects in hyperbolic metamaterials Opt Express 21 15037-47

[30] Shekhar P, Atkinson J and Jacob Z 2014 Hyperbolic metamaterials: fundamentals and applications Nano Convergence 1 1:14

[31] Savelev R S, Shadrivov I V, Yu. Kivshar Yu S 2014 Wave scattering by metal-dielectric multilayer structures with gain Pis'ma v ZhETF 100 831-36 


\section{Coherent control in planar metamaterials - Kevin F. MacDonald University of Southampton}

\section{Status}

"Coherent control' is a well-understood concept in several areas of chemistry and physics, including nanophotonics, but in the particular context of photonic metamaterials it has been established, only within the last few years, as a paradigm for dynamically controlling the manifestation of all kinds of optical phenomena in ultrathin media with exquisite precision. It has been shown that interactions between coherent light waves and thin-film media can, if the film is much thinner that the wavelength, lead to controllable energy exchange between incident and scattered waves (Fig. 1) and thereby to a plethora of new technological opportunities [1]. Though based solely on principles of linear interference, coherent control can facilitate nonlinear light-by-light control functions with $\mathrm{THz}$ bandwidth at arbitrarily low intensities [2,3]. Ultrathin media with nano-engineered optical properties unavailable in nature, i.e. planar metamaterials, can realize the full potential of this concept to impact fields including data processing and spectroscopy.

A highly absorbing plasmonic metamaterial of substantially sub-wavelength thickness positioned at a node of the standing wave light field formed by coherent counter-propagating 'control' and 'signal' beams will experience zero electric field and so will not absorb the light. Any change in the phase or intensity of the control wave that distorts the standing wave pattern, or a change in the position of the metamaterial, will break this zero-absorption regime. Indeed, at the other extreme, the metamaterial may 'perfectly coherently absorb' $100 \%$ of the incident light in both beams [1]. This effect can underpin various forms of optical switching with modulation bandwidth up to $100 \mathrm{THz}$ (limited only by resonance linewidth) [2] at extremely low (even single-photon) energy levels [3].

The effect may be harnessed in coherent four-port optical devices to deliver, without need of intrinsic optical nonlinearity, data processing functions including signal summation, inversion, and even smallsignal amplification [4], and these can be spatially multiplexed for applications such as image processing [5]. The concept can also be employed in various modes of coherent-illumination spectroscopy, for example to selectively excite and thereby disentangle electric and magnetic dipolar resonances [6].

\section{Current and Future Challenges}

Since the demonstration of saturable absorption by Vavilow and Levshin in 1926, and more so since the

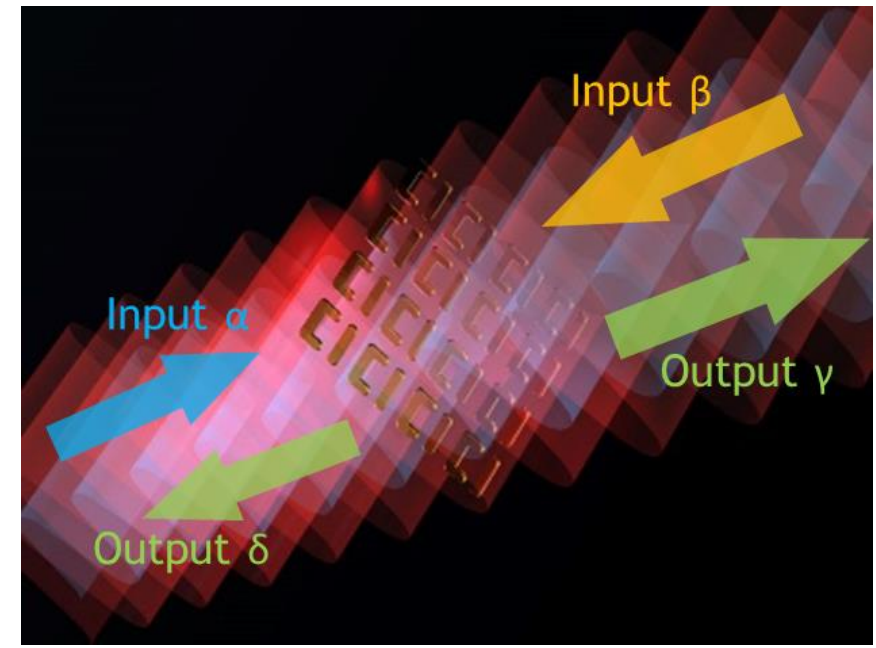

Figure 1 - Controlling the position of an ultrathin nanostructured metamaterial in the light field formed by a pair of coherent input beams, facilitates fast, high-contrast modulation of its optical properties.

invention of the laser, the research community has sought the kind of strongly nonlinear materials that can enable a variety of highly-desirable light-by-light control functions, not least for information and communications technology (ICT) network applications. These networks are existentially reliant on photonic technologies but remain largely 'opaque', with the conversion back-and-forth between optical and electronic data formats limiting bandwidth while consuming considerable energy [7].

Photonic metamaterials and metasurfaces have emerged in recent years as a platform for the realization of unusual and sharply resonant optical properties unavailable in nature via structuring at the sub-wavelength scale; for enhancing the response of conventional optically nonlinear media through hybridization; and for the engineering of extreme optical nonlinearities underpinned by reversible mechanically-, thermally-, electrically-, magnetically-, and optically-induced changes in geometric configuration at the metamolecular level.

Despite the numerous remarkable research achievements, many potential practical applications of photonic metamaterials are undermined by prohibitive energy dissipation in the metals upon which they are, thus far, usually based. For the most demanding (telecommunications) applications, this has implications for both insertion loss and control energyper-bit consumption - key metrics on which photonic technologies must compete with electronic counterparts; switching speed being another.

Realizing metamaterials with homogenously switchable, tunable and nonlinear optical properties is one thing, but thoughts are already turning to the dynamic control of metamaterials and metasurfaces at 
the level of individual metamolecules [8]. Such selectively-addressable 'random access' metamaterials would offer full active wavefront control and enable spatially multiplexed signal processing, but represent a substantial technological challenge: requiring discrete switchable elements of subwavelength size, while ideally maintaining the kind of speed and energy efficiency mentioned above.

\section{Advances in Science and Technology to Meet Challenges}

Conceptually, coherent control in planar photonic metamaterials can address many of the challenges outlined above, not least because in many applications it is reliant upon (rather than hampered by) absorption losses, and provides a mechanism for dynamically controlling them. Indeed, for the purposes of light-bylight intensity modulation, the difficulty can lie in achieving a sufficient level of single-pass absorption, or more specifically the required balance among absorption $A$, reflection $R$ and transmission $T$ : In the limit of substantially subwavelength but finite thickness, nature offers a surprisingly limited palette of optical properties. But metamaterials provide freedom to engineer the balance among $A, R$ and $T$ at will in ultrathin films, uniquely enabling for example, fourport optical devices configured to deliver small-signal amplification (cf. transistor) and signal summation/inversion (cf. logic gate) functions in wavelength bands set by design [4].

Coherent modulation is intrinsically fast and, in the absence of conventional nonlinearity, operable at arbitrarily low, even single-photon, energy levels and does not introduce harmonic distortion of signals. In being fundamentally reliant upon planar functional elements (i.e. nanostructured ultrathin films) it is also readily suited to (diffraction-limited) spatial multiplexing, opening up a range of applications in parallel signal and all-optical image processing (Fig. 2).

Most of the coherent control functionalities and potential applications considered to date rely on the interaction between an ultrathin medium and the standing wave pattern of electric field nodes and antinodes formed by a pair of collinearly-polarized incident beams. But the coexistent magnetic field standing wave should not be ignored - the spatial offset between electric and magnetic nodes in the beam propagation direction can provide for the selective excitation of electric and magnetic dipolar resonances in thin-film media [6]. Such 'excitation-selective' modes of coherent illumination offer intriguing possibilities in spectroscopy, taking advantage of the fact that the manifestation of material properties can differ between standing and travelling waves.

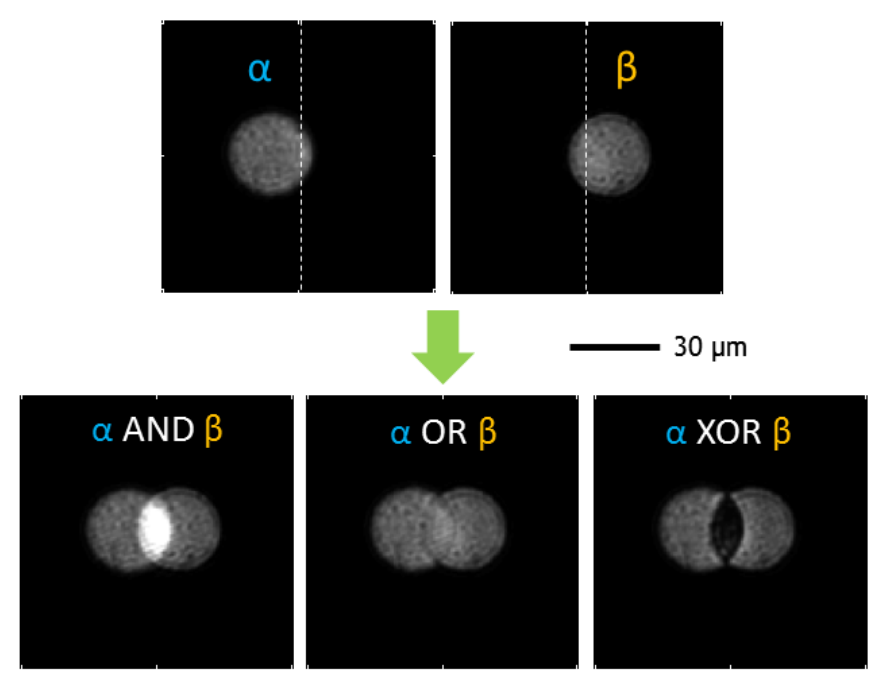

Figure $2-2 \mathrm{D}$ spatially distributed coherent control for image processing. Intersecting elements of input images $\alpha$ and $\beta$ projected onto an absorbing metasurface can be logically combined in various ways according to their mutual phase at the metasurface plane. (After Papaioannou, et al. [5].)

The coherent control paradigm of course presents its own challenges: It is based on interference and devices must therefore provide high levels of positional stability, but these are not prohibitive and may be addressed in monolithic (e.g. planar waveguide) architectures. For ICT applications, locally coherent networks are required, but such systems are already being pursued for their ability to in increase bandwidth by enabling the use of advanced data encoding formats and detection algorithms [9]. While singular coherent meta-devices may serve a variety of basic signal routing, spectroscopic and sensing applications, the requirements of complex (Boolean, conventionally electronic) data processing assemblies (cascadability, input/output isolation, etc. [10]) are challenging for any prospective all-optical platform, though coherent metadevices may present unique opportunities in alternative logical architectures [11].

\section{Concluding Remarks}

With roots in plasmonics, metamaterials and coherent optics, the concept of coherent control in planar photonic metamaterials is emerging as a powerful paradigm for manipulating the manifestation of all kinds of light-matter interactions with high contrast, at high speed and low energy, presenting a wealth of new technological opportunities.

\section{References}

1. Zhang, J., MacDonald, K.F., and Zheludev, N.I., Controlling light-with-light without nonlinearity. Light: Science \& Applications, 2012. 1: p. e18.

2. Nalla, V., et al. $100 \mathrm{THz}$ optical switching with plasmonic metamaterial. in CLEO/Europe-EQEC 2015. 2015. Munich, Germany. 
3. Roger, T., et al., Coherent perfect absorption in deeply subwavelength films in the single-photon regime. Nature Communications, 2015. 6: p. 7031.

4. Fang, X., MacDonald, K.F., and Zheludev, N.I., Controlling light with light using coherent metadevices: all-optical transistor, summator and invertor. Light: Science \& Applications, 2015. 4: p. e292.

5. Papaioannou, M., et al. Two-dimensional control of light with light on metasurfaces. Light: Science \& Applications, in press.

6. Fang, X., Tseng, M.L., Tsai, D.P., and Zheludev, N.I., Coherent excitation-selective spectroscopy of multipole resonances. Physical Review Applied, in press.

7. Miller, D.A.B., Device Requirements for Optical Interconnects to Silicon Chips. Proceedings of the IEEE, 2009. 97(7): p. 1166-1185.

8. Zheludev, N.I., Obtaining optical properties on demand. Science, 2015. 348(6238): p. 973-974.

9. Kikuchi, K., Coherent Optical Communications: Historical Perspectives and Future Directions, in High Spectral Density Optical Communication Technologies, M. Nakazawa, K. Kikuchi, and T. Miyazaki, Editors. 2010, Springer-Verlag: Berlin Heidelberg. p. 11-49.

10. Miller, D.A.B., Are optical transistors the logical next step? Nature Photonics, 2010. 4: p. 3-5.

11.Hardy, J., and Shamir, J., Optics inspired logic architecture. Optics Express, 2007. 15: p. 150-165. 


\section{Nanomechanical metamaterials - Eric Plum \\ University of Southampton}

\section{Status}

"Optical properties on demand," that is the vision behind nanomechanical photonic metamaterials which aim to provide the optical properties that we need, when and where required [1]. Potential applications range from tuneable filters, switchable gratings and variable lenses to holographic 3D displays, programmable transformation optics devices and generally adding tuneability to the various unique functionalities that metamaterials can provide.

Metamaterials may be thought of as coupled arrays of resonators, so-called meta-molecules. Therefore, dynamic control over their characteristics may be achieved by changing structural phase properties of constituent materials, controlling the way light couples to the metamaterial nanostructure or - in case of nanomechanical metamaterials - perturbing the spatial arrangement of the meta-molecules. The nanomechanical technology will be reviewed here. It exploits that the resonant optical properties of plasmonic or dielectric resonators placed within each other's near-field are strongly affected by changes of their mutual position on a length scale that is small compared to the resonant wavelength.

Starting from manually switchable [2] and magnetoelastic [3] microwave metamaterials the field of mechanical metamaterials quickly evolved to $\mathrm{THz}$ frequencies [4], where sophisticated micro-electromechanical [5] and microfluidic [6] actuation systems were developed.

However, most potential applications are in the visible and near-infrared spectral ranges which require nanomechanical metamaterials. This requires state-ofthe-art nanofabrication as well as novel ideas as sophisticated $\mathrm{THz}$ solutions are too complex to be shrunk to the optical part of the spectrum, but it also presents a great opportunity. Elastic forces and mass scale with size in a way that drives mechanical resonance frequencies of smaller structures to higher frequencies, reaching $\mathrm{GHz}$ at the sub-micron scale. Therefore, nanomechanical photonic metamaterials have the potential to be fast. At the same time, actuation mechanisms benefit from growing Coulomb, Ampère and optical forces over shrinking distances [7].

Recently, the emergence of mechanically reconfigurable nanomembrane metamaterials [8] has led to a series of breakthroughs including the demonstration of giant electro-optical [9], magnetoelectro-optical [10] and nonlinear [11] effects, optically driven modulation at up to $150 \mathrm{MHz}$ [12] and a first proof-of-principle demonstration of a selectively addressable nano-electro-mechanical metamaterial [13], see figure 1.

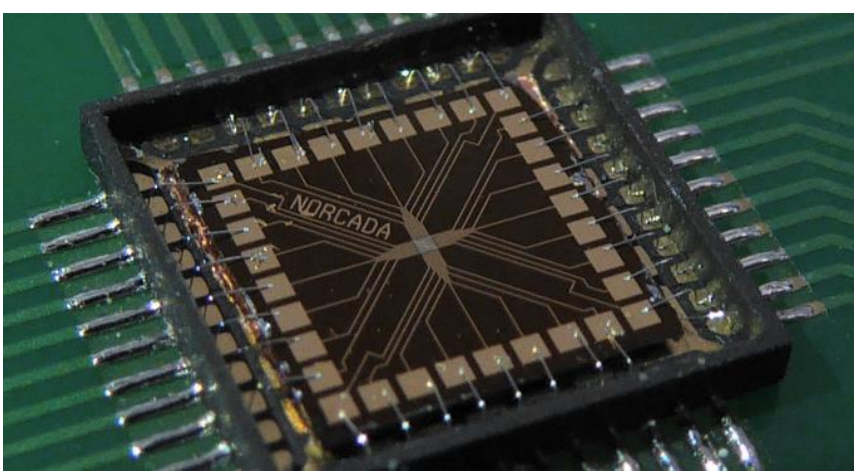

Figure 1 - Electrically addressable nanomechanical photonic metadevice [13].

\section{Current and Future Challenges}

While recent breakthroughs have demonstrated the feasibility and tremendous potential of nanomechanical photonic metamaterials driven by thermal, electric, magnetic and optical control signals, major challenges regarding performance, reliability and integration remain.

Published work introduces novel concepts, but despite record-breaking nonlinearities and electro-optical effects, current nanomechanical metamaterials are far from being optimized. Therefore, the most immediate challenge is optimization of meta-molecule designs, actuation mechanisms and material choices.

Smaller and thus faster mechanical actuators will have smaller displacements and therefore meta-molecules with higher displacement sensitivity are needed in order to achieve high-contrast changes of the metamaterial's optical properties. Solutions may be inspired by opto-mechanical systems and are likely to involve perturbation of high quality factor resonances and narrow Fano-resonances between strongly coupled resonators enabled by low-loss dielectric or plasmonic materials.

High-contrast modulation of light with nanomechanical metamaterials will also benefit from increased actuation forces and thus displacements. Larger voltages applied across smaller gaps will increase electrostatic forces leading to larger electro-optical modulation, but electrical breakdown and stiction must be avoided. Higher conductivity thin films - such as gold single crystals - would enable conductive nanomechanical structures that can support larger currents, leading to a larger magnetic Lorentz force in the presence of a magnetic field and thus larger magnetoelectro-optical modulation. Higher quality factor dielectric resonators in very homogeneous low-loss dielectric metamaterials would enable larger optical forces and thus increased nanomechanical nonlinearity. 
Also, so far materials choices have been based on what was easily available, rather than what is most desirable. Therefore, suitable materials with good optical and mechanical properties need to be identified. For example, silicon is a high index dielectric with good elastic properties, while the stiffness of diamond should lead to higher mechanical resonance frequencies.

In order to truly obtain optical properties on demand, high-contrast dynamic control of phase and intensity of light will have to be achieved with sub-wavelength spatial resolution. This will require addressing of individual reconfigurable meta-molecules on the size scale of $1 \mu \mathrm{m}^{2}$. Electronic solutions may be inspired by current display, imaging sensor and spatial light modulator technology, but the much higher pixel density will pose a challenge. An alternative approach may be diffraction-limited optical actuation of a nonlinear nano-opto-mechanical metamaterial to control its optical properties at a longer wavelength with sub-wavelength resolution.

More distant challenges are related to bringing nanomechanical photonic metamaterials to higher technology readiness levels. Here, high-yield fabrication and integration of complex nanomechanical structures and long-term reliability will be key issues that are most easily addressed by interdisciplinary teams including MEMS/NEMS experts and related industries.

\section{Advances in Science and Technology to Meet Challenges}

The progression of nanomechanical photonic metamaterials is largely a challenge of materials and nanofabrication.

Low-loss materials with either plasmonic properties or high refractive index are needed to achieve high displacement sensitivities, which depend on the quality factor of metamaterial resonances that is limited by absorption losses. Low-loss materials will also contribute to larger optical actuation forces, increased optical damage thresholds and a reduction of insertion losses, particularly the latter is needed for viable commercial applications. Therefore, the ongoing search for better plasmonic materials and the growing field of intrinsically low-loss dielectric metamaterials may be expected to drive progress.

Advances in nanofabrication are needed for highly homogeneous arrays of mechanical nanostructures (to avoid inhomogeneous broadening of resonances) and to enable the mass-production of nanomechanical photonic metadevices. While current reconfigurable photonic metamaterials are made by focused ion beam milling, a high-throughput process will need to be developed. This could be based on nanoimprint lithography followed by an etching process that frees the moving parts of the nanostructure.

Furthermore, a broad range of opportunities may arise from introducing new functional materials to nanomechanical photonic metamaterials, such as piezoelectric actuation, optical actuation using liquid crystal elastomers or isotropic modulation of optical properties based on auxetic metamaterial designs [14].

\section{Concluding Remarks}

Nanomechanical photonic metamaterials promise an unprecedented level of dynamic control over light. Modulation effects exceeding natural materials by orders of magnitude have already been reported and dramatic improvements on first proof-of-principle devices may be expected from bringing in expertise of the MEMS/NEMS, opto-mechanics and materials research communities. Ultimately, we may envisage metadevices that provide dynamic control over the wavefront of light with sub-wavelength resolution. Such devices could be programmed to perform functions from simple optical components (gratings, lenses, filters) to complex 3D holography on demand.

\section{References}

[1] Zheludev N I 2015 Obtaining optical properties on demand Science 348(6238) 973

[2] Lapine M, Powell D, Gorkunov M, Shadrivov I, Marqués R and Kivshar Y 2009 Structural tunability in metamaterials. Appl. Phys. Lett. 95084105

[3] Lapine M, Shadrivov I V, Powell D A and Kivshar Y S 2012 Magnetoelastic metamaterials. Nat. Mater. 11, 30-33

[4] Tao H, Strikwerda A C, Fan K, Padilla W J, Zhang X and Averitt R D 2009 Reconfigurable Terahertz Metamaterials. Phys. Rev. Lett. 103 147401

[5] Zhu W M, Liu A Q, Zhang X M, Tsai D P, Bourouina $\mathrm{T}$, Teng $\mathrm{J} \mathrm{H}$, Zhang $\mathrm{X} \mathrm{H}$, Guo $\mathrm{H} \mathrm{C}$, Tanoto H, Mei T, Lo G Q and Kwong D L Switchable magnetic metamaterials using micromachining processes 2011 Adv. Mater. 23, 1792-1796

[6] Zhu W M, Song Q H, Yan L B, Zhang W, Wu P C, Chin L K, Cai H, Tsai D P, Shen Z X, Deng T W, Ting S K, Gu Y D, Lo G Q, Kwong D L, Yang Z C, Huang R, Liu A Q and Zheludev N I 2015 A flat lens with tunable phase gradient by using random access reconfigurable metamaterial. Adv. Mater. 27, 4739-4743

[7] Zheludev N I and Plum E 2016 Reconfigurable nanomechanical photonic metamaterials. Nat. Nanotech. 11, 16-22

[8] Ou J Y, Plum E, Jiang L and Zheludev N I 2011 Reconfigurable Photonic Metamaterials Nano Lett. 11 2142-2144

[9] Ou J Y, Plum E, Zhang J and Zheludev N I 2013 An electromechanically reconfigurable plasmonic 
metamaterial operating in the near-infrared Nat. Nanotech. 8 252-255

[10] Valente J, Ou J Y, Plum E, Youngs I J and Zheludev N I 2015 A magneto-electro-optical effect in a plasmonic nanowire material. Nat. Commun. 6 7021

[11] Ou J Y, Plum E, Zhang J and Zheludev N I 2015 Giant nonlinearity of an optically reconfigurable plasmonic metamaterial 2015 Adv. Mater. http://dx.doi.org/10.1002/adma.201504467

[12] Karvounis A, Ou J Y, Wu W, MacDonald K F and Zheludev N I 2015 Nano-optomechanical nonlinear dielectric metamaterials $2015 \mathrm{Appl}$. Phys. Lett. 107191110

[13] Cencillo-Abad P, Ou J Y, Valente J, Plum E and Zheludev N I 2015 Randomly addressable reconfigurable photonic metamaterials in Nanometa (Seefeld, Austria)

[14] Valente J, Plum E and Zheludev N I 2015 Auxetic nanomechanical metamaterials in MRS Fall Meeting (Boston, MA, USA) 
18. Self-assembly and directed assembly for the fabrication of optical metamaterials - Xiaoying Liu and Paul F. Nealey

University of Chicago

\section{Status}

With the central theme of manipulating light at the subwavelength scale, the burgeoning field of optical metamaterials exploits novel interactions of light with substances of nanoscale features. Such artificial materials consist of patterns of structural units that are composed of multiple individual nanoscale building blocks with rationally designed geometries and arrangements. Nanostructure engineering is the basis for optical metamaterials, with large-scale manufacturing being critical toward their practical applications. To date, metamaterials have mostly been fabricated by top-down techniques, such as electron beam lithography, interference lithography, and focused-ion beam milling. These top-down fabrication methods offer good control over the location and spatial arrangement of individual features. However, they suffer from limitations in morphology (primarily two dimensional (2D)), surface roughness control, and materials scope (composition and crystallinity), that hamper optical applications. For example, energy loss caused by the inherent limit of the structural elements has severely hindered the performance of many optical metamaterials [1]. Overcoming this obstacle and improving the efficiency require the search for new materials. Colloidal nanoparticles (NPs) with a wide variety and superb control of shape, dimension, crystallinity, and surface functionality, offered by wellestablished synthetic techniques, provide an extensive, valuable library of nanoscale building blocks for the potential development of advanced metamaterials that exhibit reduced loss, broadband behavior, and/or tunable optical response. Further, the construction of various nanostructures will allow the exploration and discovery of novel light-matter interactions. To enable the design of metamaterials from colloidal NPs, it is crucial to realize precise placement and controlled assembly of individual NPs into organized arrays of nanoarchitectures with arbitrarily designed geometry and composition. Engineered self-assembly and directed assembly approaches have emerged as effective routes to NP organization and provide unique opportunities for the construction of advanced optical metamaterials.

\section{Current and Future Challenges}

Although the use of self-assembly approaches for metamaterials fabrication is just in its infancy, recent advances are beginning to reveal their potential. Examples of these studies include the utilization of block copolymer self-assembly and atomic layer

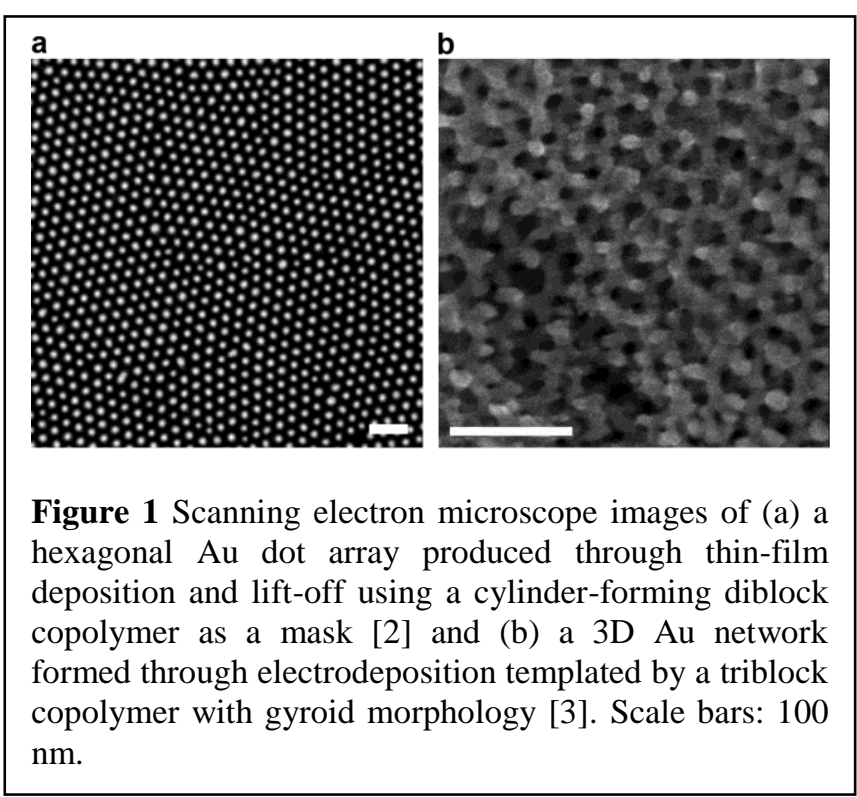

deposition to tune the light absorption properties of a 2D plasmonic array (Figure 1a) [2], the replication of a self-assembled block copolymer into a 3D $\mathrm{Au}$ nanoarchitecture that shows optical chirality (Figure 1b) [3], and the self-assembly of two types of NPs to form binary nanocrystal superlattices with tunable plasmonic coupling [4]. It is important to note that such nanopatterning, additive or subtractive, is obtained with the assistance of self-assembling materials, e.g. block copolymers and colloidal crystals. And only a limited range of materials composition and geometry could be realized. Therefore, self-assembly processes are most suitable for building metamaterials that are periodic and symmetrical and contain a minimal number of building blocks. It would greatly expand the scope of applications to incorporate different types of nanoscale building blocks into multicomponent heterostructures that exhibit orientational order, structural asymmetry, aperiodic arrangement, and/or spatial addressability.

\section{Advances in Science and Technology to Meet Challenges}

Directed assembly/self-assembly, by introducing a matrix to define the location and arrangement of individual blocks, provides a pathway to address the challenges outlined above. Such defining matrix can be designed through, for example, lithographic tools to create topographic templates or a combination of lithography and surface functionalization to generate chemical patterns. The former takes advantage of the interfacial capillary force present at the vapor-NPsubstrate three-phase contact during the evaporation of the NP suspension for site-specific placement [5]. The latter utilizes tailored interaction between pre-patterns and NPs for selective immobilization [6]. Hong et al employed a topographic template-guided assembly and self-assembly approach to generate interdigitated 


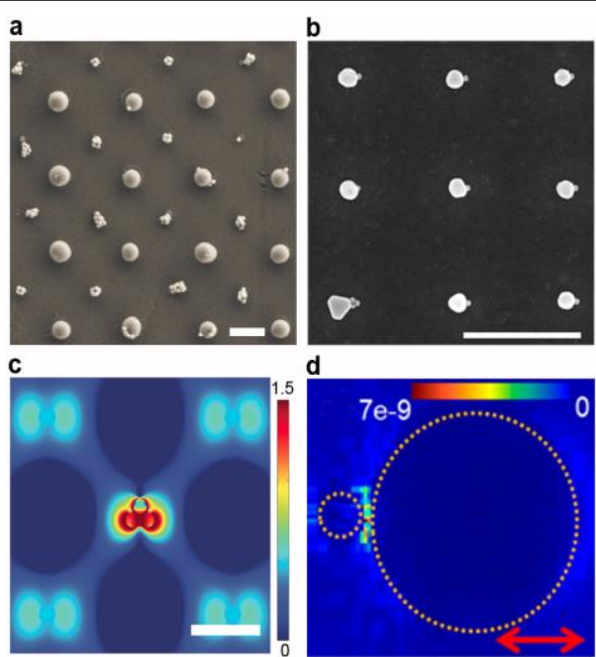

Figure 2 Scanning electron microscope images of (a) an array of $250 \mathrm{~nm} \mathrm{TiO}_{2}$ and $60 \mathrm{~nm} \mathrm{Au} \mathrm{NPs} \mathrm{through} \mathrm{a}$ topographic template-guided assembly/self-assembly [7] and (b) an array of Au heterodimers (150 and $50 \mathrm{~nm}$ ) by chemical pattern-directed assembly [8]. (c) Simulated $E$ field enhancement, $\log \left[|E|^{2} /\left|E_{0}\right|^{2}\right]$, for (a). (d) Modeled second harmonic generation intensity, $|E| /\left|E_{0}\right|$, for (b). Scale bars: (a,c) $500 \mathrm{~nm}$ and (b) $1 \mu \mathrm{m}$.

arrays of $\mathrm{TiO}_{2}$ NPs and clusters of $\mathrm{Au}$ NPs that sustain simultaneous electromagnetic interactions on multiple length scales (Figure 2a) [7]; Biswas et al developed a chemical pattern-directed assembly strategy to fabricate symmetric and asymmetric Au NP dimer arrays that support Fano resonances and nanofocusing of fundamental and second harmonic light waves (Figure 2b) [8]. These developments demonstrate the potential of directed assembly/self-assembly in overcoming the materials challenge and advancing the field of optical metamaterials. So far, reports on directed assembly of nanostructures have been predominantly limited to spherical NPs. There are great opportunities in using NPs of anisotropic shapes, such as rods and polyhedrons, which show optical response different from spheres, to expand the design space for metamaterials and enable the exploration of novel light-matter interactions.

The potential of employing the large library of ex-situ synthesized NPs has yet to be explored for the deterministic construction of advanced optical metamaterials, both 2D and 3D. A combination of topdown and bottom-up design philosophy in essence, directed assembly/self-assembly present exciting opportunities toward overcoming the fabrication hurdles, expanding the structure/material scope, and enabling large-area manufacturing of metamaterials. To advance this research direction in order to meet the requirements of various technological applications, researchers will need to establish the knowledge and tools required to build arbitrarily designed architectures, especially multicomponent hybrid structures and genuinely 3D materials. Such tools may include, for example, sequential, programmable construction based on molecular recognition [9] and orientation and ordering of NPs mediated by liquid crystals or block copolymers [10]. The precise localization of each building block relies on the fundamental understanding and fine control of the pattern-particle and particle-particle interactions. These interactions, such as hydrogen bonds, electrostatic forces, and chemical bonds, afford substantial flexibility to direct the particle organization process through engineering the surface functionalities of patterns and NPs. Furthermore, the expertise that has accumulated in the past decade on self-assembly in colloidal systems provides a valuable knowledge base that can be translated into the tailoring of the particleparticle interactions. Additionally, this field will greatly benefit from the development of patterning methods, especially those with feature location and arrangement encoded in molecular systems, which would enable more precise NP placement and scalable fabrication of the confining matrix.

\section{Concluding Remarks}

Through a combination of top-down fabrication of the confining matrix and bottom-up assembly/selfassembly of nanoscale building blocks, directed assembly/self-assembly is poised to provoke innovations for deterministic construction of optical metamaterials. It is imperative that we focus the future efforts of this relatively new research direction on designing technologically relevant metamaterials that show desired functions and performance, such as nanofocusing of electromagnetic fields, optical chirality, and negative refraction index. Its advancement will require the close partnership of researchers and interchange of expertise from multiple disciplines, such as chemistry, engineering, physics, and theory, with potential areas of focus including computation-assisted design of metamaterials with customized optical properties, nanomaterials engineering for programmable assembly, and process control for improved macroscopic uniformity.

\section{References}

[1] Khurgin J B 2015 Nature Nano. 10 2-6

[2] Haegglund C, Zeltzer G, Ruiz R, Thomann I, Lee HB-R, Brongersma M L and Bent S F 2013 Nano Lett. 13 3352-7

[3] Vignolini S, Yufa N A, Cunha P S, Guldin S, Rushkin I, Stefik M, Hur K, Wiesner U, Baumberg J $\mathrm{J}$ and Steiner U 2012 Adv. Mater. 24 OP23-7

[4] Ye X, Chen J, Diroll B T and Murray C B 2013 Nano Lett. 13 1291-97

[5] Stebe K J, Lewandowski E and Ghosh M 2009 Science 325 159-60

[6] Onses M S and Nealey P F 2013 Small 9 4168-74 
[7] Hong Y, Qiu Y, Chen T and Reinhard B M 2014 Adv. Func. Mater. 24 739-46

[8] Biswas S, Liu X, Jarrett J W, Brown D, Pustovit V, Urbas A, Knappenberger K L, Nealey P F and Vaia R A 2015 Nano Lett. 15 1836-42

[9] Hellstrom S L, Kim Y, Fakonas J S, Senesi A J, Macfarlane R J, Mirkin C A and Atwater H A 2013 Nano Lett. 13 6084-90

[10] Balazs A C, Emrick T and Russell T P 2006 Science 314 1107-10 


\section{Nanoparticles for complex multimaterial nanostructures - Cherie R. Kagan and Christopher B. Murray University of Pennsylvania}

\section{Status}

Nanoparticles (NPs) are artificial building blocks for the construction of optical metamaterials that operate in the visible to near-to-mid infrared regions of the electromagnetic spectrum. Metal NPs were first studied in the Au sols of Faraday and later recognized to be the origin of the colors of stained glass. Advances in chemical synthetic methods now allow the controlled preparation of colloidal, plasmonic NPs from metals ${ }^{1}$ [Fig. 1a-c] and highly-doped semiconductors ${ }^{2,3}$ [Fig. 1d-f]. The NPs may be tailored in size $(2-200 \mathrm{~nm}$ in diameter), shape, ${ }^{4,5}$ and composition to tune the carrier concentration, carrier relaxation, and dielectric constant and therefore the spectral position and width of the plasmonic resonance and the spatial distribution of resulting electrical fields.

These colloidal NPs may be self- or directed ${ }^{11,12}$ - to assemble from solution to create metamaterials from a single type of NP or from a combination of two- or even three-types of NPs [Fig. 1g,h]. ${ }^{6}$ The composition, concentration, organization, and orientation of the NPs in the assemblies allows control over the number and types of interactions between NPs. The spacing between NPs and therefore the strength of interparticle interactions can be tuned by selecting or exchanging the surface ligand chemistry used during or introduced after synthesis [Fig. 2a]. For example, reducing the interparticle spacing to $<1 \mathrm{~nm}$ through ligand exchange of $\mathrm{Au} \mathrm{NP}$ assemblies gives rise to an insulator-to-metal transition seen in the dielectric function, as collective interactions between neighboring NPs gives rise to meso-scale properties [Fig. 2b,c]. ${ }^{7}$ Engineering assemblies from NP combinations allows the design of multimaterials with properties not found in bulk.

Colloidal NPs may be printed and patterned to form subwavelength, NP-based superstructures also referred to as "meta-atoms." Meta-atoms constructed from NP assemblies allow metamaterials and two-dimensional metasurfaces to be designed hierarchically, taking advantage of the properties of the NP assemblies and the size, shape, and composition of the superstructures. For example, nanoimprinted NP-based metasurfaces operate as filters with bandstops tunable with the diameter of the meta-atoms [Fig. 2d-f] $]^{7}$ and, in more complex designs constructed from different size and shape superstructures, control polarization of transmitted light ${ }^{13}$. Metamaterials and metasurfaces may be fabricated over large-area using solution-based printing techniques.

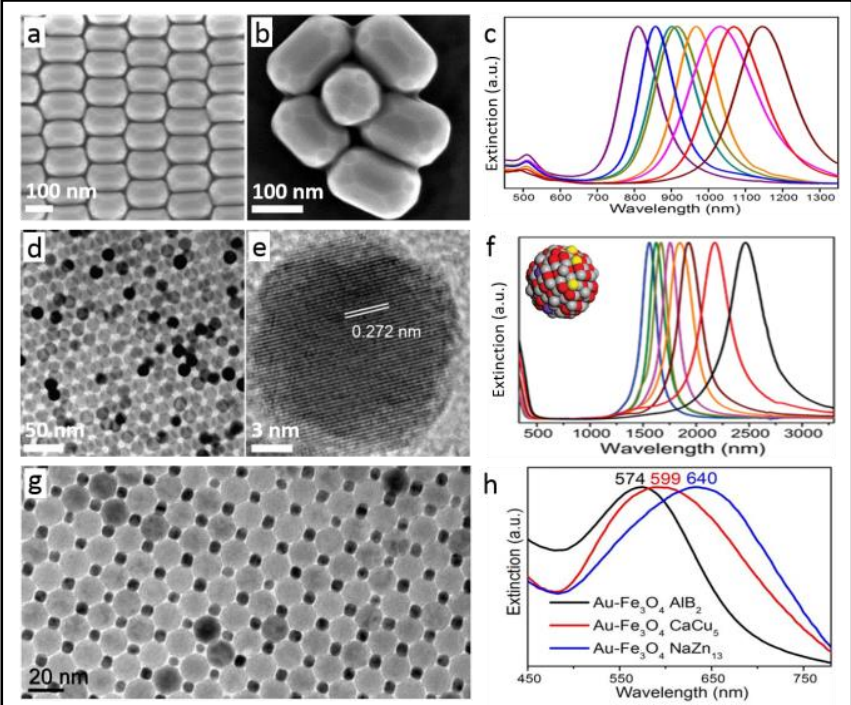

Fig. 1 (a) Low- and (b) high-resolution SEM images and (c) aspect ratio-dependent (3.8:1 to 7.5:1) extinction spectra of Au nanorods. (d) Low- and (e) high-resolution TEM images and (f) doping concentration-dependent extinction spectra of CdO:F,In NPs. (g) TEM image and (h) structuredependent extinction spectra of $\mathrm{Au}-\mathrm{Fe}_{3} \mathrm{O}_{4}$ binary NP superlattices.

\section{Current and Future Challenges}

NPs may be synthesized with near-atomic precision, but they have residual inhomogeneities in size, shape, and composition. As colloids they are electrostatically or sterically stabilized by one type or a cocktail of surface ligands, in relatively large concentration given the high NP surface-to-volume ratio. The high NP surface-to-volume ratio also makes their optical properties susceptible to shifts upon exposure to gases or liquids, which can be detrimental in some devices, but can be exploited in sensors. Most computational treatments do not incorporate the atomistic nature of the NPs and their surfaces. Advances in structural and optical characterization are enabling interrogation of isolated NPs, but not close-packed NP ensembles, and chemical analyses do not yet have the needed sensitivity.

In NP assemblies, the structural and optical designs are intimately connected through the NP size, shape, and packing, making it difficult to independently adjust the optical properties of and the coupling between NPs. The structure of most assemblies reported to date have been dominated by entropic energy terms (space filling), but the ability to create open frameworks or arbitrary symmetries is desirable. The average interparticle distance in assemblies can be controlled with sub-nanometer precision, however disorder, defects, and grain boundaries broaden the materials properties. We note that if the distance between grain boundaries is large, then they may be excluded in patterned, subwavelength meta-atoms. 
For the NP assembly to appear homogeneous to the electromagnetic field and for the field from plasmonic NPs to strongly influence neighboring NPs, the NPs must be in proximity. As the wavelength of operation is moved to the blue, smaller NPs are needed and loss typically increases. At sub-nanometer interparticle distances accessible through ligand exchange, strong NP coupling arises from quantum mechanical interactions and commonly used classical models of plasmonic materials breakdown.

The size, shape, position, and placement of meta-atoms is limited by variations common to the lithographic method used in fabrication. Also the solution-based NP deposition may affect meta-atom morphology and shrinkage upon ligand exchange may lead to larger distances between meta-atoms. To date, meta-atoms have only been reported with simple shapes and compositions and therefore have limited function.

\section{Advances in Science and Technology to Meet Challenges}

NP synthesis and purification methods continue to push toward the preparation of true molecular building blocks, with deeper mechanistic understanding of NP nucleation and growth and with improved purification through automation and in-situ optical monitoring. The greater spatial and energy resolution offered by aberration-corrected electron microscopy is revealing chemical structure and optical function at near atomic dimensions in NPs. Super-resolution optical microscopies are reaching to below $50 \mathrm{~nm}$, approaching that needed to characterize more closelypacked NP assemblies.

Independent manipulation over the structure and properties of NP assemblies and the assembly of more open frameworks is possible by engineering the NP surface charge and the composition and arrangement of ligands; for example to create patchy NPs and to introduce molecular recognition for directional bonding. The combination of top-down fabrication and bottom-up assembly allows the directed-assembly of NPs in fabricated templates. Size- and shape-selective templates enable the position and orientation of different NPs to be controlled; for example to realize heterodimers of Au nanorods and hexagonal prismatic, upconverting nanophosphors that show polarizationdependent enhancement of luminescence [Fig. $2 \mathrm{~g}-\mathrm{i}$ ] ${ }^{8}$

Strategies to manage loss in NP assemblies may follow similar approaches to those in bulk by introducing alloys or gain compensation, ${ }^{9}$ but here through the synthesis of NP alloys and the co-assembly of different NPs. While NP surfaces may introduce scattering, tailoring the surface chemistry and faceting may allow the design of "electron waveguides" that limit loss.
Quantum-mechanical simulations and corrections to classical electrodynamics are capturing the physics introduced at sub-nanometer interparticle distances. ${ }^{10}$ Descriptions of the pair-wise and larger ensemble interactions between NPs is needed to allow the inverse design of NP-based multimaterials for their application specific device integration. Controlling the composition and spacing between different NPs may enable the dielectric function of NP assemblies to be tailored from that characteristic of the bulk to that of a diluted metal with epsilon near zero.

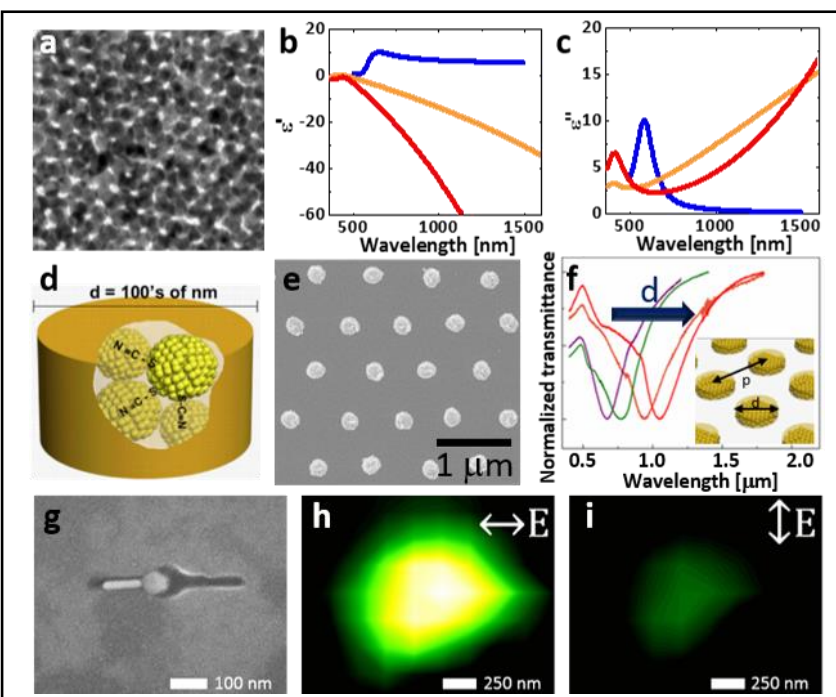

Fig. 2 a,b) TEM image of thiocyanate (SCN)-exchanged $\mathrm{Au}$ $\mathrm{NC}$ films. c) real and d) imaginary dielectric function of oleic acid-capped (blue) and SCN-exchanged (orange) Au NC films and bulk Au (red). d) Schematic, e) SEM image, and transmittance spectra of nanoimprinted and SCNexchanged Au NC-based nanopillar arrays $(d=120-300 \mathrm{~nm})$. g) SEM image of a Au nanorod-nanophosphor heterodimer and h,i) the polarization-dependent enhanced emission.

The development of solution-based printing and patterning techniques will allow the fabrication of meta-atoms that have more complex shapes and that integrate multiple NP materials, structured laterally and vertically, to construct optical metamaterials and metasurfaces with device functions. For example, meta-atoms could be patterned to introduce anisotropy or chirality that may not be present in the individual building blocks and thereby engineer the propagation and polarization of light.

\section{Concluding Remarks}

The synthesis and programmed assembly of colloidal NPs enables the design of metamaterials with properties not found on the periodic table. For example, controlling the size and shape of the NPs and the interparticle spacing allows the dielectric function of NP-based metamaterials to be engineered. However advanced structural, optical, and computational methods are needed to characterize NP assemblies at atomic scales. Patterning the NP assemblies into meta- 
atoms allows the properties of NP-based materials to be exploited in devices.

The integration of multiple NP materials will lead to new functions. For example, the co-assembly or layering of plasmonic and magnetic NPs will allow the construction of magnetoplasmonic multimaterials. Selecting NPs and intervening ligands that are responsive to external stimuli (e.g. light, heat, electric fields) will enable the fabrication of reconfigurable metamaterials. Fundamental understanding of NP properties and couplings will open a new era based on inverse design of NP-based multimaterials for devices.

\section{References}

(1) Rycenga, M.; Cobley, C. M.; Zeng, J.; Li, W.; Moran, C. H.; Zhang, Q.; Qin, D.; Xia, Y. Controlling the Synthesis and Assembly of Silver Nanostructures for Plasmonic Applications. Chem. Rev. 2011, 111, 3669-3712.

(2) Ye, X.; Fei, J.; Diroll, B. T.; Paik, T.; Murray, C. B. Expanding the Spectral Tunability of Plasmonic Resonances in Doped Metal-Oxide Nanocrystals through Cooperative Cation-Anion Codoping. J. Am. Chem. Soc. 2014, 136, 11680-11686.

(3) Buonsanti, R.; Milliron, D. J. Chemistry of Doped Colloidal Nanocrystals. Chem. Mater. 2013, 25, 1305-1317.

(4) Ye, X.; Zheng, C.; Chen, J.; Gao, Y.; Murray, C. B. Using Binary Surfactant Mixtures to Simultaneously Improve the Dimensional Tunability and Monodispersity in the Seeded Growth of Gold Nanorods. Nano Lett. 2013, 13, 765-771.

(5) Lohse, S. E.; Murphy, C. J. The Quest for Shape Control: A History of Gold Nanorod Synthesis. Chem. Mater. 2013, 25, 1250-1261.

(6) Ye, X.; Chen, J.; Diroll, B. T.; Murray, C. B. Tunable Plasmonic Coupling in Self-Assembled Binary Nanocrystal Superlattices Studied by Correlated Optical Microspectrophotometry and Electron Microscopy. Nano Lett. 2013, 13, 12911297.

(7) Fafarman, A. T.; Hong, S.-H.; Caglayan, H.; Ye, X.; Diroll, B. T.; Paik, T.; Engheta, N.; Murray, C. B.; Kagan, C. R. Chemically Tailored Dielectric-toMetal Transition for the Design of Metamaterials from Nanoimprinted Colloidal Nanocrystals. Nano Lett. 2013, 13, 350-357.

(8) Greybush, N. J.; Saboktakin, M.; Ye, X.; Della Giovampaola, C.; Oh, S. J.; Berry, N. E.; Engheta, N.; Murray, C. B.; Kagan, C. R. Plasmon-Enhanced Upconversion Luminescence in Single Nanophosphor-Nanorod Heterodimers Formed through Template-Assisted Self-Assembly. ACS Nano 2014, 8, 9482-9491.
(9) Boltasseva, A.; Atwater, H. A. Materials Science. Low-Loss Plasmonic Metamaterials. Science 2011, 331, 290-291.

(10) Esteban, R.; Borisov, A. G.; Nordlander, P.; Aizpurua, J. Bridging Quantum and Classical Plasmonics with a Quantum-Corrected Model. Nat. Commun. 2012, 3, 825.

(11) J. Fan, K. Bao, L. Sun, J. Bao, V. N. Manoharan, P. Nordlander, F. Capasso, "Plasmonic Mode Engineering with Templated Self-Assembled Nanoclusters," Nano Letters 2012, 12, 5318-5324.

(12) M. B. Ross, J. C. Ku, V. M. Vaccarezza, G. C. Schatz, C. A. Mirkin, Nanoscale form dictates mesoscale function in plasmonic DNA-nanoparticle superlattices, Nature Nano, 2015, 10, 453-458.

(13) W. Chen, M. Tymchenko, P. Gopalan, X. Ye, Y. Wu, M. Zhang, C. B. Murray, A. Alu, and C. R. Kagan, "Large-Area Nanoimprinted Colloidal $\mathrm{Au}$ Nanocrystal-Based Nano-antennas for Ultrathin Polarizing Plasmonic Metasurfaces," NANO Letters, 2015, 15 (8), 5254-5260. 


\section{Eutectics as metamaterials, plasmonic materials and photonic materials}

- Dorota A. Pawlak

Institute of Electronic Materials Technology and University of Warsaw

\section{Status}

Eutectics are composite materials, when in a solid state, with two or more phases which self-organize after cooling down a completely mixable melt of a eutectic composition. As composites they can possess additive properties dependent on the intrinsic properties of the single phases and product properties, which arise from combining the two phases together in a particular structure. They are most well known for having a lower melting point than either of the two single phases, and due to this property they are often used in every-day life. Eutectics in solid-state form are monolith and multiphase materials and the following properties are interesting for metamaterials: (i) they can exhibit various geometrical motifs; (ii) the structure refinement can be tuned at the micron to nanometer scale; (iii) they are highly crystalline; (iv) various combinations of component phases may possibly be obtained; (v) the interfaces can be very sharp - even atomically smooth with precise crystallographic relation to each other; (vi) after growth they can be post-engineered in many ways.

Metal-metal eutectic alloys have been studied for many years due to their improved mechanical properties, and oxide-oxide eutectics more recently due to their high strength and resistance at high temperatures and pressures. Metal-oxide eutectics with high refractory metals such as tungsten and molybdenum, often exhibiting a structure of metallic microfibers embedded in an oxide matrix, were studied in the 1970s as electron emitters. Eutectics are also studied as materials for energy applications such as solid oxide fuel cells and photoanodes in photoelectrochemical cells. Recently, it has been proposed that eutectics with fibrous geometries could be used as photonic crystals [1] and that, due to eutectic product properties, eutectic solidification could be one of the ways of manufacturing metamaterials $[2,3,4]$.

\section{Current and Future Challenges}

It is not easy to adapt the concept of metamaterials in materials science to enable large scale $[5,6]$ applications, for example manufacturing utilizing the self-organization mechanism in eutectics. It is due to the intrinsic properties of materials prepared with a bottom-up approach, which inevitably includes some degree of disorder in the structure. That is why the potential applications can only be those which can

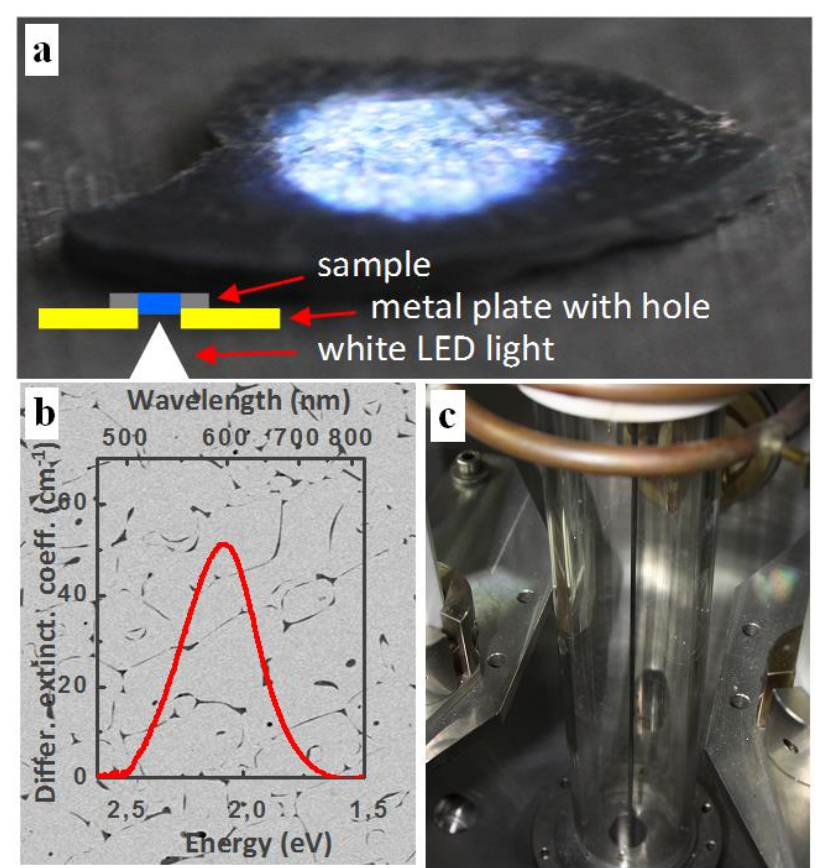

Figure 1. Eutectic-based $\mathrm{Bi}_{2} \mathrm{O}_{3}-\mathrm{Ag}$ nanoplasmonic volumetric composite. (a) Blue light transmitted through the material due to the (b) LSPR at $\sim 595 \mathrm{~nm}$. The eutectic rod in the heart of the micro-pulling down apparatus [7].

accommodate some degree of disorder and yet not lose the expected

functionality. One example is the localized surface plasmon resonance, LSPR, which primarily depends on the chemical composition, size and shape of the metallic precipitates and on their environment. A bulk nanoplasmonic material manufactured utilizing a $\mathrm{Bi}_{2} \mathrm{O}_{3}-\mathrm{Ag}$ eutectic composite has been recently demonstrated [7], with a LSPR in the yellow wavelength range, $\sim 600 \mathrm{~nm}$. In Fig. 1a the blue light transmitted through the sample is shown, while the complementary yellow one is being absorbed and/or scattered. The original eutectic structure, Fig. 1b, is here modified by annealing which promotes formation of $\mathrm{Ag}$ and $\mathrm{Bi}$ nanoparticles within the composite. The LSPR is tunable here with annealing conditions such as atmosphere, temperature and time [8]. The properties of the original material can be enhanced, e.g., when doped with $\mathrm{Re}^{3+}$ ions the luminescence is enhanced [7].

Eutectic materials can exhibit various geometrical motifs, where the regular eutectic structures are fibrous (rodlike), Fig. 3a,b [2, 9] and lamellar (layered) Fig. $3 c$. However, other structures are possible such as globular, spiral or broken-lamellar. It has been shown that even a split-ring-resonator geometry, which is pivotal for metamaterials, can be obtained during the growth of a $\mathrm{SrTiO}_{3}-\mathrm{TiO}_{2}$ eutectic, Figs. 3 d-f [3].

The fibrous and lamellar eutectic structures could be the most easy to use as metamaterials, since such 
properties as subwavelength transmission and hyperbolic dispersion could be obtained, and they are less sensitive to structural disorder.

The majority of the metamaterials properties are available by combining two materials with opposite signs of permittivity, which is typically achieved by manufacturing a metal-dielectric material. However, utilizing eutectics enables applying various component phases, for example, the negative permittivity in one of the two phases could be achieved by using phonons, plasmons, or excitons. Thus, it is relatively easy in a eutectic to achieve the wavelength range where one phase would exhibit $\operatorname{Re}(\varepsilon)>0$ and the other phase $\operatorname{Re}(\varepsilon)<0$.

This property has been used and a subwavelength transmission has been demonstrated theoretically utilizing phonon polaritons in fibrous alkali halides eutectics formed by close-packed arrangements of aligned microrods of one phase embedded in another phase [10]. Also, with the fibrous or lamellar eutectic structure it should be relatively easy to achieve hyperbolic dispersion. With the effective medium approach a range of frequencies of hyperbolic dispersion has been predicted in alkali halides eutectics in the $\mathrm{THz}$ wavelength range, for $\mathrm{LiF}$ rods embedded in $\mathrm{KCl}$ or $\mathrm{NaCl}[11,12]$. Since the hyperbolic dispersion range changes with the structure refinement, it is thus possible to shift it by applying different growth rate. Various material combinations were proposed enabling potential covering of the $5-20 \mathrm{THz}$ wavelength range with proposed eutectics [5]. With higher growth rate the structure refinement in eutectics is increasing enabling size control from micron to nanometer scale.

Utilizing eutectics for metamaterials or plasmonic materials is in the early stages, thus future challenges include experimental demonstration of properties like hyperbolic dispersion, negative refraction, subwavelength transmission and others. This is in addition to utilizing different resonant properties such as LSPR together with the intrinsic properties of the other component phase, or special chemical elements, enabling optical activity or optical nonlinearities in order to demonstrate enhancement of these properties and their potential application in various fields. Eutectics as optical materials in general have been little investigated and this is a fertile field for research. Since it is much easier to obtain dielectric-dielectric eutectics it is also a challenge to use them in this field utilizing the eutectic structuring and the intrinsic properties of these materials including phononic and excitonic resonances as well as various properties such as the Faraday effect, superconductivity [13], optical activity, nonlinearities [14] and many others.
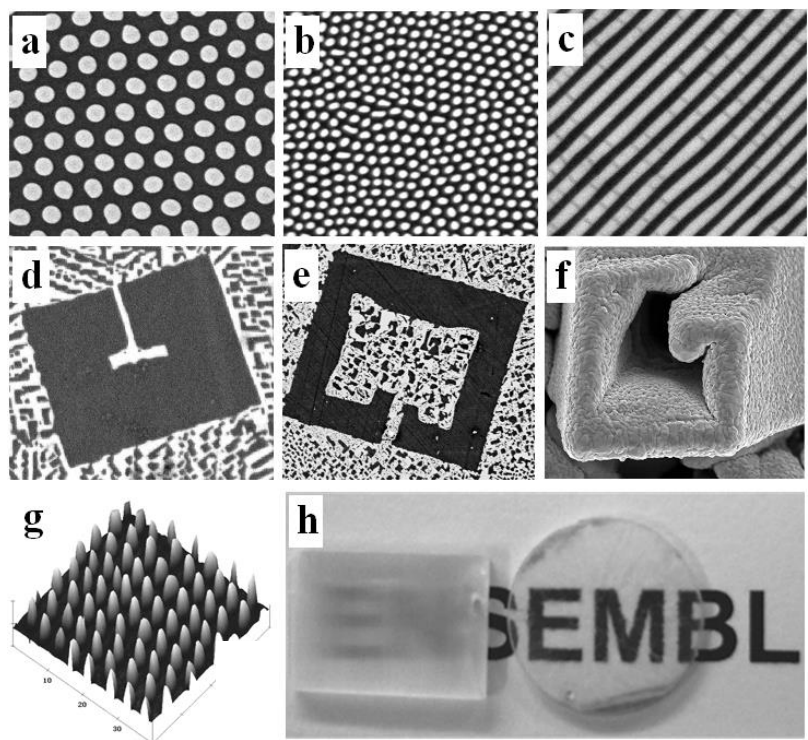

Figure 2. Fibrous (a-b) [2], and lamellar [6] (c) eutectic geometries pertinent to hyperbolic metamaterials, and (d, e, f) [3] pivo " "r metamaterials split-ring-resonatorlike geometrius sbtained by eutectic solidification. Structured surfaces $(\mathrm{g})$ of selectively etched eutectic [2], and (h) transparent eutectics of well aligned fibrous structure, copied with permission from [10].

\section{Advances in Science and Technology to Meet Challenges}

Crystallizing a eutectic we can obtain various geometrical motifs often almost periodically ordered. One of the key issues is the potential to design first the material, and that is why the development of numerical methods which would enable prediction of the obtained eutectic structure is necessary $[15,16]$. A eutectic grown in standard conditions will typically exhibit regions of eutectic grains, often hundreds of micrometers in extent, where the micro/nanostructure is the same. One of the technological challenges is to obtain a single grain eutectic. There are several ways of manufacturing metal-dielectric eutectics: (i) directly from the melt, (ii) by selective etching an oxide-oxide or halide-halide eutectic and infiltrating it with metal, or (iii) by preparing a eutectic with one phase easily reducible to a metal. However, not many phase diagrams are known, especially with good plasmonic metals like silver, and this should be investigated; and secondly, not many attempts have been performed yet to prepare metal-dielectric eutectics with other methods. The other potential area for investigation is to dope the eutectics with metallic nanoparticles, which could enter only one of the phases or both of them depending on the intrinsic properties of the materials such as surface tension in the liquid phase. Structure dimensions on the nanoscale are also a challenge for eutectic fabrication since control at this length scale is not easily obtained. The ways to obtain nanostructured eutectics include: (i) fast cooling, (ii) addition of another phase, (iii) growth in a small volume which 
could enable fast cooling, (iv) devitrification of eutectic glasses, and (v) potentially, the addition of nanoparticles. Other new approaches include combining the eutectic growth with other manufacturing methods, like obtaining eutectics in photonic crystals templates [17].

\section{Concluding Remarks}

Eutectics and metamaterials appeared finally at a crossroad in terms of their applications. This is a new approach which can only be successful when material scientists and theoreticians working in electromagnetism cooperate efficiently. From just a few groups in the world working on this subject, it started to spread worldwide with the European Project ENSEMBLE 2008-2012 (www.ensemble-fp7.eu), national projects as TEAM (Polish Science Foundation), HARMONIA (National Science Center of Poland) and recently in newly-launched MURI Project in US.

\section{Acknowledgements}

DAP would like to thank HARMONIA and PRELUDIUM projects (National Science Centre of Poland, 2013/10/M/ST5/00650, 2012/07/N/ST5/02428), NoE Project (Air Force Office for Scientific Research, 14RT0477), NMP-FP7 ENSEMBLE Project (European Commission), and TEAM project (Polish Science Foundation), for supporting the work on eutectics for photonics, metamaterials and plasmonics.

\section{References}

[1] Pawlak, D. A.; Lerondel, G.; Dmytruk, I.; Kagamitani, Y.; Durbin, S.; Fukuda, T. Second order self-organized pattern of terbium-scandiumaluminum garnet and terbium-scandium perovskite eutectic. J. Appl. Phys. 2002, 91, 9731.

[2] Pawlak, D. A., Kolodziejak, K., Turczynski, S., Kisielewski, J., Rozniatowski, K., Diduszko, R., Kaczkan, M., Malinowski M. Self-organized, rodlike, micron-scale microstructure of $\mathrm{Tb}_{3} \mathrm{Sc}_{2} \mathrm{Al}_{3} \mathrm{O}_{12^{-}}$ $\mathrm{TbScO}_{3}: \mathrm{Pr}$ eutectic. Chem. Mat. 2006, 18, 24502457.

[3] Pawlak, D. A., Turczynski, S. Gajc, M., Kolodziejak, K., Diduszko, R., Rozniatowski, K., Smalc, J., Vendik I., How far are we from making metamaterials by self-organization? The microstructure of highly anisotropic particles with an SRR-like geometry. Adv. Funct. Mat. 2010, 20, 1116-1124.

[4] Pawlak, D. A. Self-organized structures for metamaterials, Handbook of Artificial Materials, Editor in Chief: F. Capolino, ISBN10: 1420054236, Taylor \& Francis, 2009.

[5] Merino, R. I., Acosta, M. F., Orera, V. M. New polaritonic materials in the $\mathrm{THz}$ range made of directionally solidified halide eutectics, J. Eur. Ceram. Soc., 2014, 34, 2061-2069.

[6] Bienkowski, K. Turczynski, S. Diduszko, R. Gajc, M. Gorecka, E. Pawlak, D. A. Growth of a plateshaped $\mathrm{SrTiO}_{3}-\mathrm{TiO}_{2}$ eutectic Cryst. Growth Des. 2011, 11, 3935-3940.

[7] Sadecka, K., Gajc, M., Orlinski, K., Surma, H. B., Klos, A., Jozwik-Biala, I., Sobczak, K., Dluzewski, P., Toudert, J, Pawlak, D. A., When eutectics meet plasmonics: nanoplasmonic, volumetric, selforganized, silver-based eutectic, Adv. Opt. Mat. 2015, 3, 381-389.

[8] Sadecka, K.; Toudert, J.; Surma, H. B.; Pawlak, D. A. Temperature and atmosphere tunability of the nanoplasmonic resonance of a volumetric eutecticbased $\mathrm{Bi}_{2} \mathrm{O}_{3}-\mathrm{Ag}$ metamaterial. Opt. Express 2015, 23, 19098-19991.

[9] Pawlak, D. A., Kolodziejak, K., Rozniatowski, K., Diduszko, R, Kaczkan, M., Malinowski, M., Piersa, M., Kisielewski, J., Lukasiewicz, T. $\mathrm{PrAlO}_{3^{-}}$ $\operatorname{PrAl}_{11} \mathrm{O}_{18}$ eutectic: its microstructure and spectroscopic properties. Cryst. Growth and Design 2008, 8, 4, 1243.

[10] Myroshnychenko, V. Stefanski, A. Manjavacas, A. Kafesaki, M. Merino, R. I. Orera, V. M. Pawlak, D. A. Garcia de Abajo, F. J. Interacting plasmon and phonon polaritons in aligned nano- and microwires. Opt. Express 2012, 20, 10879-10887.

[11] Reyes-Coronado, A., Acosta, M. F., Merino, R. I., Orera, V. M., Kenanakis, G., Katsarakis, N., Kafesaki, M., Mavidis, Ch., Garcia de Abajo, J., Economou, E. N., Soukoulis, C. M. Selforganization approach for $\mathrm{THz}$ polaritonic metamaterials, Optics Express, 2012, 20 1466314682.

[12] M. Massaouti, A. A. Basharin, M. Kafesaki, M. F. Acosta, R. I. Merino, V. M. Orera, E. N. Economou, C. M. Soukoulis, S. Tzortzakis, Eutectic epsilonnear-zero metamaterial terahertz waveguides, Optics Letters, 2013, 38, 1140-1142.

[13] Shiroka, T.; Fittipaldi, R.; Cuoco, M.; Renzi, R. De.; Maeno, Y.; Lycett, R. J.; Ramos, S.; Forgan, E. M.; Baines, C.; Rost, A.; Granata, V.; Vecchione A. $\mu S R$ studies of superconductivity in eutectically grown mixed ruthenates. Phys. Rev. B 2012, 85, 134527-134534.

[14] Simon, F.; Clevers, S.; Gbabode, G.; Couvrat, N.; Agasse-Peulon, V.; Sanselme, M.; Dupray, V.; Coquerel, G. Enhanced second harmonic generation from an organic self-assembled eutectic binary mixture: A case study with 3-nitrobenzoic and 3,5dinitrobenzoic acids. Cryst. Growth \& Design 2015, 15, 946-960.

[15] Rátkai, L.; Szállás, A.; Pusztai, T.; Mohri, T.; Gránásy, L. Ternary eutectic dendrites: Pattern formation and scaling properties. J. Chem. Phys. $2015,142,154501$. 
[16] Akamatsu, S.; Plapp, M. Eutectic and peritectic solidification patterns. Curr. Opin. Solid STate Mater. Sci. 2015 article in press.

[17] Kim, J.; Aagesen, L. K.; Choi, J. H.; Choi, J. Kim, H. S.; Liu, J.; Cho, Ch-R.; Kang, J. G.; Ramazani, A.; Thornton, K.; Braun P. V. Template-directed directionally solidified three-dimensionally mesostructured $\mathrm{AgCl}-\mathrm{KCl}$ eutectic photonic crystals. Adv. Mat. 2015, 27, 4551-4559. 
21. Self-assembled

hyperbolic metamaterials - Igor I. Smolyaninov $^{1}$ and Vera N. Smolyaninova ${ }^{2}$

${ }^{1}$ University of Maryland

${ }^{2}$ Towson University

\section{Status}

Hyperbolic metamaterials were originally introduced to overcome the diffraction limit of optical imaging [1]. Soon thereafter it was realized that hyperbolic metamaterials demonstrate a number of novel phenomena resulting from the broadband singular behavior of their density of photonic states [2]. These novel phenomena and applications include super resolution imaging [3], enhanced quantumelectrodynamic effects [4], thermal hyperconductivity [5] and new stealth technology [6]. The nature of this singularity in hyperbolic metamaterials can be understood from the density of photonic states expressed in terms of the phase space volume enclosed by two surfaces corresponding to different values of the light frequency. For extraordinary waves in a uniaxial dielectric metamaterial, the dispersion law

$$
\frac{k_{I I}^{2}}{\varepsilon_{\perp}}+\frac{k_{\perp}^{2}}{\varepsilon_{I I}}=\frac{\omega^{2}}{c^{2}}
$$

describes an ellipsoid in the momentum space if both components of the dielectric permittivity tensor are positive. The phase space volume enclosed between two such ellipsoids at different light frequencies is then finite, corresponding to a finite density of photonic states. However, when one of the components of the dielectric permittivity tensor is negative, eq.(1) describes a hyperboloid in the phase space. As a result, the phase space volume between two such hyperbolloids (corresponding to different values of frequency) is infinite, leading to an infinite density of photonic states. This behavior is robust: while disorder can change the magnitude of the dielectric permittivity components, leading to a deformation of the corresponding hyperboloid in the momentum space, it will remain a hyperboloid and will therefore still support an infinite density of states.

The opposite sign of the dielectric permittivity tensor components also leads to another extremely interesting feature of hyperbolic metamaterials. Extraordinary light rays propagating inside a hyperbolic metamaterial look similar to particle world lines in a $2+1$ dimensional Minkowski spacetime [2]. This analogy leads to gravity-like nonlinear optical interactions in hyperbolic metamaterials [7], while thermal fluctuations in self-assembled hyperbolic metamaterials exhibit some similarity to cosmological

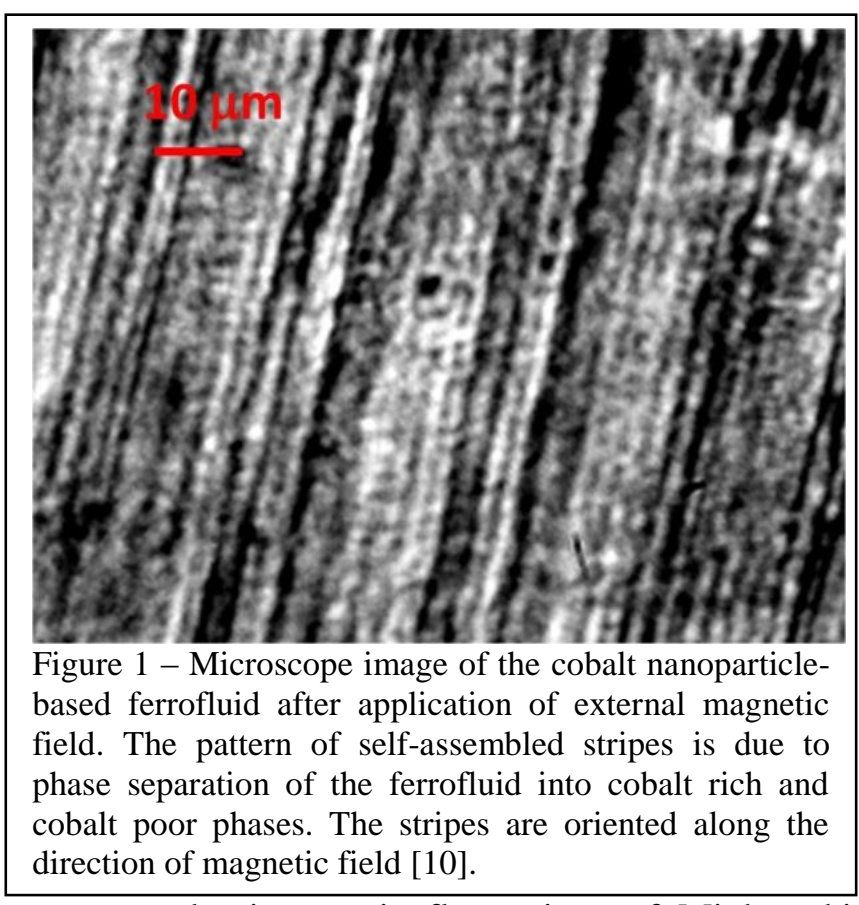

systems and microscopic fluctuations of Minkowski spacetime [8].

\section{Current and Future Challenges}

Unfortunately, considerable difficulties still exist in fabrication of three-dimensional hyperbolic metamaterials, which limit their scientific and device applications described above. Because of considerable limitations of currently used 3D nanofabrication techniques, hyperbolic metamaterials are typically confined to small sizes in two spatial dimensions. Therefore, recent demonstration that a ferrofluid subjected to a strong enough magnetic field may exhibit hyperbolic metamaterial properties [8,9] opens up many new directions in hyperbolic metamaterial research. A typical example of such a self-assembled hyperbolic metamaterial is shown in Figure 1. For our experiments we have chosen cobalt magnetic fluid 270001 from Strem Chemicals composed of $10 \mathrm{~nm}$ cobalt nanoparticles in kerosene coated with sodium dioctylsulfosuccinate and a monolayer of LP4 fatty acid condensation polymer. The average volume fraction of cobalt nanoparticles in this ferrofluid is $8.2 \%$. Cobalt behaves as an excellent metal in the long wavelength infrared range (LWIR): the real part of its refractive index, $\mathrm{n}$, is much smaller than its imaginary part, $\mathrm{k}$. Thus, real part of $\varepsilon$, Re $\varepsilon=\mathrm{n}^{2}-\mathrm{k}^{2}$, is negative, and its absolute value is much larger than its imaginary part, $\operatorname{Im} \varepsilon=2 \mathrm{nk}$. Therefore, it is highly suitable for fabrication of hyperbolic metamaterials. Since such a metamaterial is created by $3 \mathrm{D}$ self-assembly, its dimensions are not limited by nanofabrication issues. Unlike other typical metamaterial systems, such a macroscopic self-assembled 3D hyperbolic 
metamaterial may also exhibit reach physics associated with topological defects and phase transitions. Therefore, as was pointed out recently by Mielczarek and Bojowald [10] the properties of self-assembled magnetic nanoparticle-based hyperbolic metamaterials exhibit strong similarities with the properties of some microscopic quantum gravity models, such as loop quantum cosmology. On the other hand, while scientifically interesting due to such analogies, topological defects need to be avoided in device applications of the self-assembled hyperbolic metamaterials. Indeed, 3D self-assembly of macroscopic metamaterial samples unavoidably leads to formation of various defects in the bulk of the metamaterial, which may affect its optical properties.

\section{Advances in Science and Technology to Meet Challenges}

It appears that our self-assembly approach enables fabrication of large size three-dimensional hyperbolic metamaterials having relatively low-loss, broadband performance in the long wavelength infrared (LWIR) frequency range. Moreover, metamaterial properties are easily tunable by external magnetic field, while metamaterial sample size in all three dimensions is limited only by the dimensions of magnet used. Moreover, the same technique is applicable to fabrication of 3D photonic hypercrystals [9]. Similar to hyperbolic metamaterials, photonic hypercrystals exhibit broadband divergence in their photonic density of states due to the lack of usual diffraction limit on the photon wave vector. On the other hand, similar to photonic crystals, the dispersion law of extraordinary photons in photonic hypercrystals is modulated by forbidden gaps near the boundaries of the photonic Brillouin zones. The reason for such modulation is that a diluted ferrofluid develops very pronounced phase separation into cobalt rich and cobalt poor phases if subjected to a strong enough external magnetic field. Unique spectral properties of photonic hyper-crystals lead to extreme sensitivity of the material to monolayer coatings of cobalt nanoparticles, which should find numerous applications in biological and chemical sensing. We have combined our 3D self-assembled fabrication approach with well-established Fourier Transform Infrared (FTIR) spectroscopy techniques, and explored sensing potential of the newly developed photonic hypercrystal-based FTIR spectroscopy, as illustrated in Figure 2. Since LWIR range is well known as a "chemical fingerprint" region, this research direction may find numerous applications in homeland security. We anticipate that the developed techniques may also be applied to other magnetic nanoparticlebased ferrofluids. For example, gold or silver coated magnetic nanoparticles may be used to shift the hyperbolic frequency range towards near infrared and

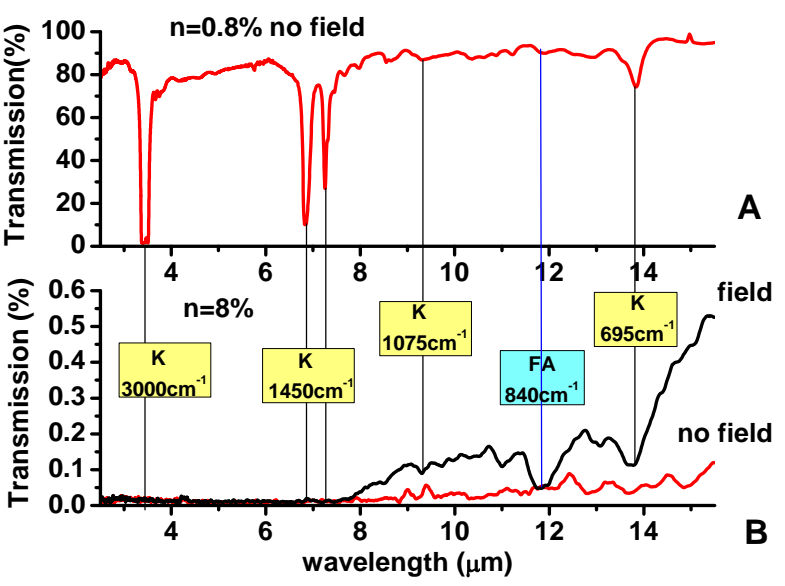

Figure 2 - (a) FTIR transmission spectrum of diluted ferrofluid exhibits clear set of kerosene absorption lines. (b) Transmission spectra of the ferrofluid measured with and without application of external magnetic field. Magnetic field induced transmission spectrum contains a pronounced absorption line at $840 \mathrm{~cm}^{-1}$, which can be attributed to a monolayer coating of lactic acid. Kerosene absorption lines are marked with yellow boxes, while the fatty acid line at $840 \mathrm{~cm}^{-1}$ is marked with a green box.

visible ranges, where nanofabrication of 3D hyperbolic metamaterials and photonic hypercrystals appears to be the most challenging [11].

\section{Concluding Remarks}

We have demonstrated that three dimensional selfassembly of hyperbolic metamaterials and photonic hyper-crystals by application of external magnetic field to a magnetic nanoparticle-based ferrofluid appears to be a viable way to overcome difficulties presented by standard 3D nanofabrication techniques. Moreover, the electromagnetic properties of such self-assembled hyperbolic metamaterials are broadly tunable by the magnetic field, which is difficult to achieve by other means. However, hyperbolic nature of this system is fluctuational in nature: thermal fluctuations of the magnetic particle concentration may locally change the sign of one of the components of the permittivity tensor of the system, so strong enough magnetic field is needed for this approach. Unique spectral properties of hyperbolic metamaterials lead to extreme sensitivity of the material to monolayer coatings of cobalt nanoparticles, which should find numerous applications in biological and chemical sensing. On the other hand, using such large scale 3D hyperbolic metamaterial samples as Minkowski spacetime analogs, we may be able to experimentally explore such theoretically predicted phenomena as subwavelength spatial solitons [7] and the recently proposed space-time cloaks. 
This work was supported in part by NSF grant DMR1104676.

\section{References}

[1] Z. Jacob, L. V. Alekseyev, and E. Narimanov, "Optical hyperlens: Far-field imaging beyond the diffraction limit", Optics Express 14, 8247 - 8256 (2006).

[2] I. I. Smolyaninov and E. E. Narimanov. "Metric signature transitions in optical metamaterials", Phys.Rev. Lett. 105, 067402 (2010).

[3] I. I. Smolyaninov, Y. J. Hung, and C. C. Davis, "Magnifying superlens in the visible frequency range", Science 315, 1699-1701 (2007).

[4] Z. Jacob, I. I. Smolyaninov, and E. E. Narimanov, "Broadband Purcell effect: Radiative decay engineering with metamaterials", Appl. Phys. Lett. 100, 181105 (2012).

[5] E. E. Narimanov and I. I. Smolyaninov, "Beyond Stefan-Boltzmann law: Thermal hyperconductivity", arXiv:1109.5444

[6] E. E. Narimanov, H. Li, Y. A. Barnakov, T. U. Tumkur, and M. A, Noginov, "Darker than black: Radiation-absorbing metamaterial", arXiv:1109.5469

[7] I. I. Smolyaninov, "Analogue gravity in hyperbolic metamaterials”, Phys. Rev. A 88, 033843 (2013).

[8] I. I. Smolyaninov, B. Yost, E. Bates, and V. N. Smolyaninova, "Experimental demonstration of metamaterial "multiverse" in a ferrofluid", Optics Express 21, 14918-14925 (2013).

[9] V. N. Smolyaninova, B. Yost, D. Lahneman, E. Narimanov, and I. I. Smolyaninov, "Self-assembled tunable photonic hyper-crystals", Scientific Reports 4, 5706 (2014).

[10] M. Bojowald, J. Mielczarek, "Some implications of signature-change in cosmological models of loop quantum gravity", arXiv:1503.09154 [gr-qc]

[11] E. Narimanov, "Contol of emission and absorption with metamaterials", Roadmap Article in this issue of Journal of Optics. 
22. Large area, roll-to-roll processing of optical metamaterials - Debashis Chanda CREOL, University of Central Florida

\section{Status}

Embedding dissimilar materials (metal/dielectric) over large area in multi-layer architecture at nano-scale is still a technological challenge. Large area metamaterials open up completely new paradigm in photonics with engineered properties like negative refractive index, near-zero index, artificial magnetism etc. All present conventional lithographic techniques like electron and ion beam lithography provide the necessary sub-micron resolution and excellent control over the in-plane geometries. However, its practical use is restricted to severely limited pattern area ${ }^{1,2}$. This size limitation, taken together with the complexity of the lithography tools and their extremely slow patterning speeds, make these techniques poorly suited to requirements for realistic applications where cost, throughput, area coverage and long range uniformity are important considerations. Alternative approaches such as those based on colloidal self-assembly ${ }^{3,4}$, interference lithography 5, and two photon polymerization based direct laser writing ${ }^{6}$ lack the ability to embed composite metal/dielectric layers. Nanoimprint lithography and various forms of soft lithography ${ }^{3,7,8}$, in their standard embodiments, offer the necessary resolution, but they do not form multilayer composite structures. There is a technological void in multi-layer large area nanomanufacturing of composite architectures.

\section{Current and Future Challenges}

The key technological challenge is how to fabricate large area metamaterials following simple roll-to-roll type processes for practical applications. In our recent works we demonstrated a novel nanotransfer printing technique where multi-layer metal/dielectric stack was grown on a patterned silicon stamp and subsequently the stack was transfer printed to a suitable flexible/rigid substrate for near-infrared ${ }^{9}$ and visible ${ }^{10}$ operations as illustrated in Fig. 1a. The technique is directly applicable to fabrication of metal/dielectric composite 2D ("metasurfaces") as well as 3D ("3D metamaterials") geometries with excellent structural uniformity, in ways that are scalable to arbitrarily large areas and compatible with manufacturing. We demonstrated fabrication of 3D optical domain negative index metamaterials with 11 alternating metal/dielectric layers (30-50 nm thick) and submicron unit cell dimensions (80-200 nm), over areas > $75 \mathrm{~cm}^{2}$, corresponding to $>10^{5} \times 10^{5}$ unit cells, all with excellent uniformity and minimal defects as shown in Fig. 1b-c ${ }^{9,10}$. These areas and numbers of unit cells both correspond to increases of more than $2 \times 10^{7}$
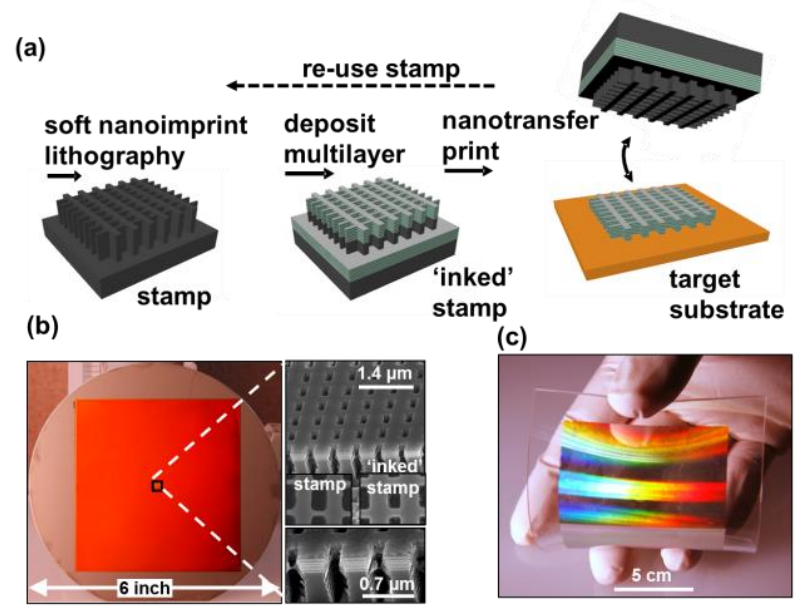

(c)

Fig. 1. (a) Schematic illustration of the three basic steps of nanotransfer printing technique. Nanotransfer Printed metamaterial stack. (b) 'Inked' stamp and (c) corresponding printed example 11-layer $\mathrm{Ag} / \mathrm{MgF}_{2}$ stack on flexible plastic sheet for near-infrared operation.

[D. Chanda et. al., (Cover Article) Nature

Nanotechnology, 6, 402-407, 2011].

times, over previous reports based on focused ion beam based 3D nanopatterning ${ }^{1,2}$. A single cycle of printing with a single stamp demonstrate use in a manufacturing mode at throughputs that are $\sim 10^{8}$ times higher than those possible with state-of-the-art ebeam/FIB systems $(\sim 2.5 \mathrm{~s} \text { per unit cell })^{1,2}$.

To develop and extend the nanotransfer printing technique for industry scale large area nanomanufacturing of photonic/electronic components, we identified few areas which need further refinement. One of the key issues is transfer of the device stack from the stamp to a suitable substrate in a high throughput manner. Typically a controlled transfer mechanism is needed to avoid micro-cracking. Also transfer of fine feature size $(<200 \mathrm{~nm})$ multi-layer pattern is challenging.

\section{Advances in Science and Technology to Meet Challenges}

The nanotransfer printing technique requires automated control systems to consistently avoid micro-cracking of large sheet of connected pattern during transfer of material stacks to foreign substrates. The technique is more suitable for isolated patterns where discrete features can be transfer printed to a rigid or flexible substrate without any micro-cracking concerns. As a follow up work, we developed a complementary approach in which a nanoimprinted polymeric pattern (stamp) served as scaffold to grow 3D material stacks 11. This scheme does not involve any transfer printing step. Instead, the shape of the pattern is governed by interstitial space of the imprinted scaffold. Material grown on top of the scaffold is removed and discarded following a physical lift-off process. A subsequent oxygen plasma etching step removes the polymeric transient stamp, leaving behind the multi-layer stack on 
(a) Nanoimprint lithography
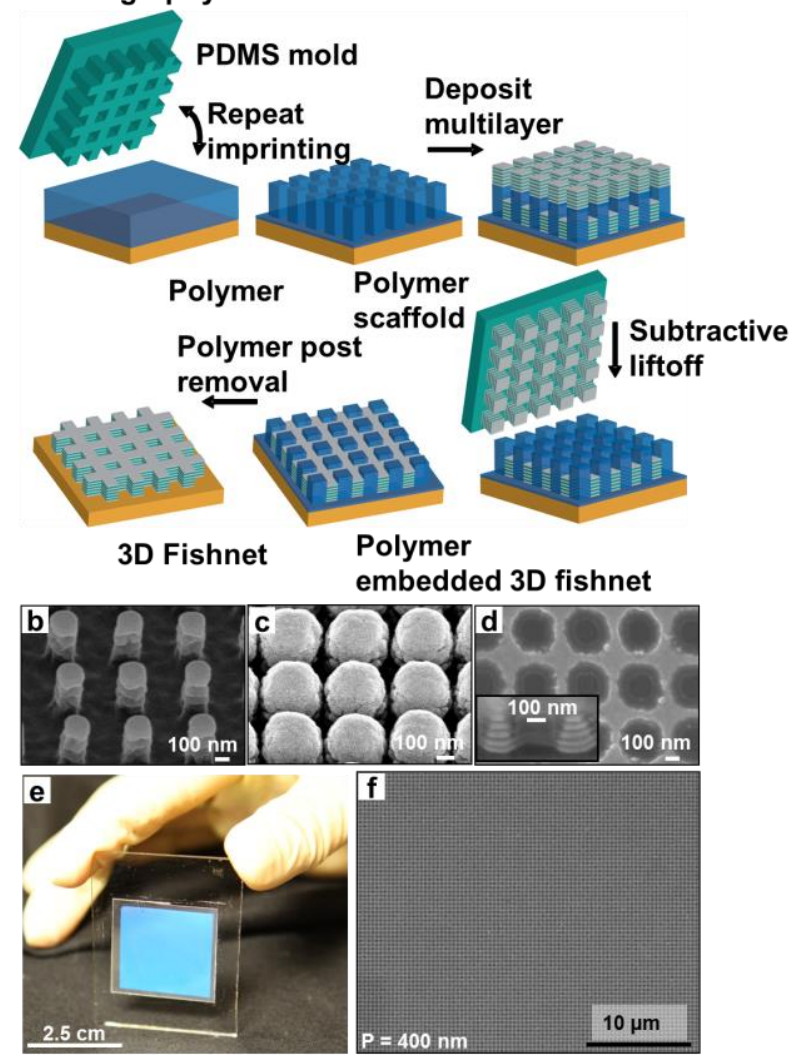

Fig. 2. (a) Schematic overview of the imprinted multilayer metamaterial fabrication technique. SEM of (b) imprinted polymer stamp, (b) 'Inked' stamp and (f) corresponding printed example 11-layer $\mathrm{Ag} / \mathrm{MgF}_{2} \mathrm{NIM}$ stack for visible operation. The corresponding macroscopic optical image is shown in (e). [D. Chanda et. al., ACS Nano, 6, 5535 -5542, 2014]

the substrate. By comparison to nanotransfer printing ${ }^{9}$, this technique offers improved robustness in operation, primarily because the stack remains untouched throughout the process; only unwanted material from the top of the scaffold is removed. The subsequent removal of the transient soft polymeric stamp by $\mathrm{O}_{2}$ plasma etch completes the process. Schematic overview of the imprinted multilayer metamaterial fabrication technique is illustrated in Fig. 2a. The SEM of (b) imprinted polymer stamp, (b) 'Inked' stamp and (f) corresponding printed example 11-layer $\mathrm{Ag} / \mathrm{MgF}_{2}$ stack for visible operation is shown in Fig. 2. The corresponding macroscopic optical image is shown in Fig. 2e.

The imprinted technique depends on a very simple approach based on a transient polymeric stamp where arbitrary shape of the interstitial space of the stamp governs the geometry of the physically evaporated stack. The complementary revised method offers much improved robustness in operation, primarily because the stack remains untouched throughout the process; only unwanted material is removed from the top of the

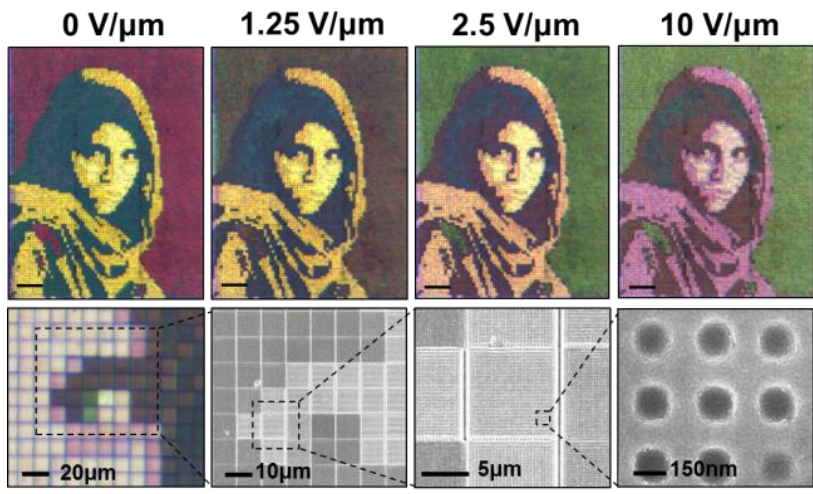

Fig. 3. Full-color polarization independent metasurface display. [D. Chanda et. al., (Featured Article) Nature Communications, Vol. 6, pp. 7337, 2015.]

In this work, we report an increase in the tuning ability spanning the entire visible spectrum for the first time through the use of a highly birefringent liquid crystal (LC) and a periodic array of imprinted shallow aluminium nanowells that allow complete LC reorientation and maximum overlap between the plasmonic fields and LC. To emphasize the display potential of these metasurfaces, the resultant color palette is exploited to form color tunable images. Figure 3 (top) shows $0.75 \mathrm{~mm} \times 1 \mathrm{~mm}$ reflective optical images of the Afghan Girl (National Geographic Society), while the singular sample is tuned through $0-10 \mathrm{~V} \mu \mathrm{m}^{-1}$ of applied electric field. The $10 \mu \mathrm{m}$ x $10 \mu \mathrm{m}$ pixels can be seen in the following SEM subfigures. In this example, the pixelated metasurface is singularly addressed and therefore limited to display one image. Further addressing of each pixel based on a standard addressing scheme will enable the display of video.

\section{Concluding Remarks}

Due to the simplicity and robustness of the process steps, the nanotransfer printing ${ }^{9,10}$ and imprinting ${ }^{11}$ techniques are suitable for high throughput nanomanufacturing of newer classes of nanophotonic/electronic devices. Both techniques are well suitable for scalability based on a roll-to-roll process on rigid as well as flexible and curved surfaces as shown in our previous works $9,10,12,13$. Due to ease of release from the stamp, the nanotransfer printing is more suitable for connected patterns whereas isolated patterns are relatively easy to subtract in the imprinting technique. In combination, both of these techniques offer unique large area metamaterial design opportunities which are previously missing. In building these structures, there is no unnecessary complexity, which at the end means that the structure contains a very low defect density, and thus exhibits strong optical/electrical properties based on any arbitrary geometry and material combinations. Such processes can be translated to roll-to-roll processing which means 
that the entire fabrication process can be scaled to factory norms.

\section{References}

1 J. Valentine, S. Zhang, T. Zentgraf, E. Ulin-Avila, D.A. Genov, G. Bartal \& $\mathrm{X}$. Zhang,"Three-dimensional optical metamaterial with a negative refractive index". Nature 455, 376-380 (2008).

2 C. Garcia-Meca, J. Hurtado, J. Marti \& A. Martinez,"Low-Loss Multilayered Metamaterial Exhibiting a Negative Index of Refraction at VisibleWavelengths". Phys. Rev. Lett. 106 (2011).

3 Y. Xia, J. A. Rogers, K. E. Paul \& G.M. Whitesides,"Unconventional Methods for Fabricating and Patterning Nanostructures". Chem. Rev. 99, 18231848 (1999).

4 A. Blanco, E. Chomski, S. Grabtchak, M. Ibisate, S. John, S. W. Leonard, C. Lopez, F. Meseguer, H. Miguez, J. P. Mondia, G. A. Ozin, O. Toader \& H. M. van Driel,"Large-scale synthesis of a silicon photonic crystal with a complete three-dimensional bandgap near 1.5 micrometres". Nature 405, 437-440 (2000).

5 M. Campbell, D. N. Sharp, M. T. Harrison, R. G. Denning \& A. J. Turberfield,"Fabrication of photonic crystals for the visible spectrum by holographic lithography". Nature 404, 53-56 (2000).

6 M. Deubel, G. Von Freymann, M. Wegener, S. Pereira, K. Busch \& C. M. Soukoulis,"Direct laser writing of three-dimensional photonic-crystal templates for telecommunications". Nature Materials 3, 444-447 (2004).
8

A. Costner, M. W. Lin, W. L. Jen \& C. G. Willson,"Nanoimprint Lithography Materials Development for Semiconductor Drvice Fabrication". Annu. Rev. Mater. Res. 39, 155-180 (2009).

9 D. Chanda, K. Shigeta, S. Gupta, T. Cain, A. Carlson, A. Mihi, A. J. Baca, G. R. Bogart, P. V. Braun \& J. A. Rogers,"Large-area flexible 3D optical negative index metamaterial formed by nanotransfer printing". Nature Nanotechnology 6, 402-407 (2011).

10 L.Gao, Y.Kim, A.Vazquez-Guardado, K.Shigeta, S.Hartanto, D.Franklin, C.J. Progler, G.R.Bogart, J. A. Rogers \& D. Chanda,"Materials Selections and Growth Conditions for Large Area Multilayered Visible Negative Index Metamaterials Formed by Nanotransfer Printing". Advanced Optical Materials 2, 256-261 (2014).

11 Li Gao, Kazuki Shigeta, Abraham Vazquez-Guardado, C.J. Progler, G.R.Bogart, J.A.Rogers \& D. Chanda,"Nanoimprinting Techniques for Large-Area Three-Dimensional Negative Index Metamaterials with Operation in the Visible and Telecom Bands". ACS Nano 6, 5535 -5542 (2014).

12 Daniel Franklin, Yuan Chen, Abraham Vazquez-Guardado, Sushrut Modak, Javaneh Boroumand, Daming Xu, ShinTson Wu \& Debashis Chanda,"Polarization Independent, Actively Tunable Color Generation on Imprinted Plasmonic Surfaces". Nature Communications In Press (2015).

13 Li Gao, Kazuki Shigetac, Abraham Vazquez-Guardadoa, C.J. Progler, G.R.Bogart， J.A.Rogers \& Debashis Chanda,"Nanoimprinting Techniques for Large-Area Three-Dimensional Negative Index Metamaterials with Operation in the Visible and Telecom 
Bands". ACS Nano 6, 5535-5542 (2014). 